Prepared for the U.S. Department of Energy under Contract DE-AC05-76RL01830

\title{
Laboratory Studies on Surface Sampling of Bacillus anthracis Contamination: Summary, Gaps, and Recommendations
}

\author{
GF Piepel \\ BG Amidan
}

$\mathrm{R} \mathrm{Hu}$

November 2011

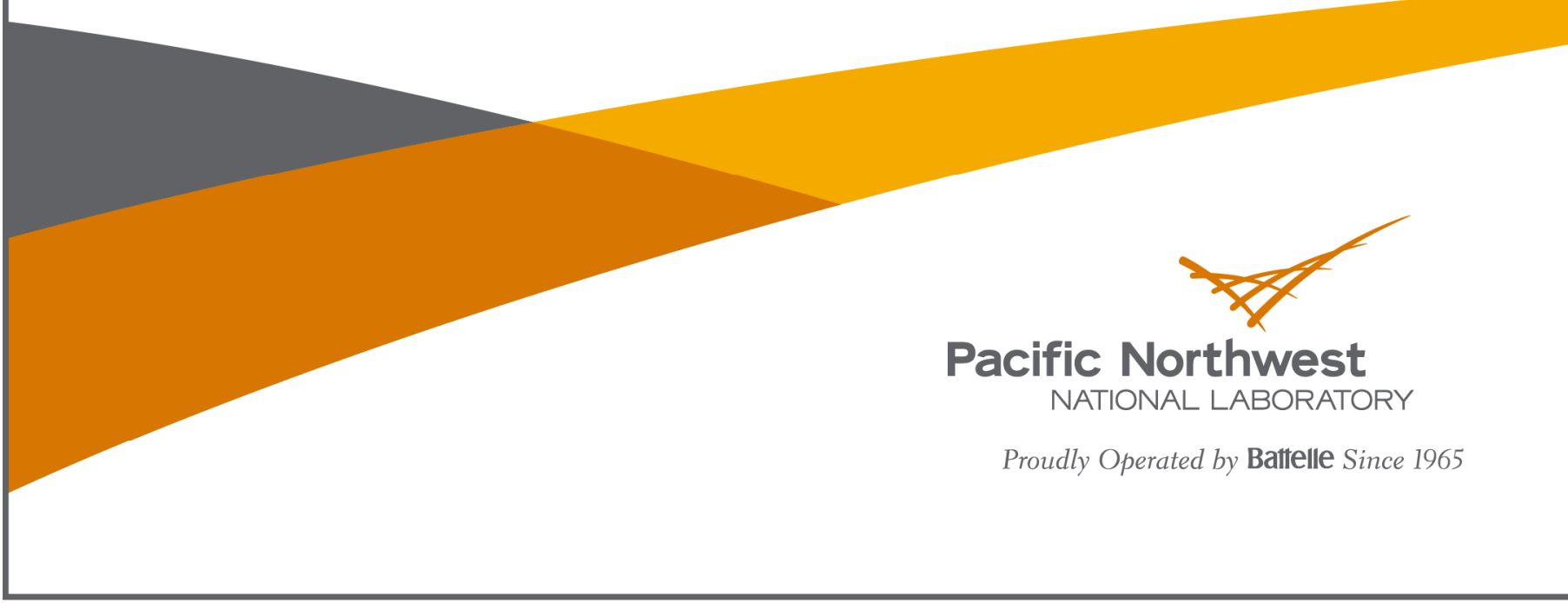




\title{
DISCLAIMER
}

This report was prepared as an account of work sponsored by an agency of the United States Government. Neither the United States Government nor any agency thereof, nor Battelle Memorial Institute, nor any of their employees, makes any warranty, express or implied, or assumes any legal liability or responsibility for the accuracy, completeness, or usefulness of any information, apparatus, product, or process disclosed, or represents that its use would not inf ringe privately owned rights. Reference herein to any specific commercial product, process, or service by trade name, trademark, manufacturer, or otherwise does not necessarily constitute or imply its endorsement, recommendation, or favoring by the United States Government or any agency thereof, or Battelle Memorial Institute. The views and opinions of authors expressed herein do not necessarily state or reflect those of the United States Government or any agency thereof.

\author{
PACIFIC NORTHWEST NATIONAL LABORATORY \\ operated by \\ BATTELLE \\ for the \\ UNITED STATES DEPARTMENT OF ENERGY \\ under Contract DE-AC05-76RL01830
}

Printed in the United States of America
Available to DOE and DOE contractors from the
Office of Scientific and Technical Information
P.O. Box 62, Oak Ridge, TN 37831-0062;
ph: (865) 576-8401
fax: $(865) 576-5728$
email: reports@adonis.osti.gov

Available to the public from the National Technical Information Service

5301 Shawnee Rd., Alexandria, VA 22312

ph: (800) 553-NTIS (6847)

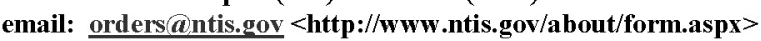

Online ordering: http://www.ntis.gov

This document was printed on recycled paper. 
PNNL-20910

Unclassified

\section{Laboratory Studies on Surface Sampling of Bacillus anthracis Contamination: Summary, Gaps, and Recommendations}

GF Piepel

BG Amidan

$\mathrm{R} \mathrm{Hu}$

November 2011

Prepared for

the U.S. Department of Energy

under Contract DE-AC05-76RL01830

Pacific Northwest National Laboratory

Richland, Washington 99352 



\section{Executive Summary}

This report summarizes previous laboratory studies to characterize the performance of methods for collecting, storing/transporting, processing, and analyzing samples from surfaces contaminated by Bacillus anthracis or related surrogates. The focus is on plate culture and count estimates of surface contamination for swab, wipe, and vacuum samples of porous and nonporous surfaces. Summaries of the previous studies and their results were assessed to identify gaps in information needed as inputs to calculate key parameters critical to risk management in biothreat incidents. One key parameter is the number of samples needed to make characterization or clearance decisions with specified statistical confidence. Other key parameters include the ability to calculate, following contamination incidents, the 1) estimates of Bacillus anthracis contamination, as well as the bias and uncertainties in the estimates, and 2) confidence in characterization and clearance decisions for contaminated or decontaminated buildings. Gaps in knowledge and understanding identified during the summary of the studies are discussed. Recommendations are given for future evaluations of data from existing studies and possible new studies. 



\section{Acknowledgments}

The Pacific Northwest National Laboratory (PNNL) work summarized in this report (prepared in the format required for subsequent journal submission) was funded by the Biological Research and Development Branch of the Chemical and Biological Division in the Science and Technology Directorate of the U.S. Department of Homeland Security (DHS). We also acknowledge the U.S. Department of Energy's Young Women in Science program, which funded the work of student intern Rebecca Hu. PNNL is a multiprogram national laboratory operated for the U.S. Department of Energy by Battelle under Contract DE-AC05-76RL01830.

The authors acknowledge Jayne Morrow (NIST), Paula Krauter (SNL), Landon Sego (PNNL), and Brent Pulsipher (PNNL) for reviewing a draft of this report. We also thank the authors of several publications we cited who provided 1) copies of presentations or pre-publication copies of articles, 2) additional data or calculations, 3) additional information not included in their publications, and 4) feedback on initial tabular summaries of their work. These include Jamie Almeida (NIST), Mark Buttner (University of Nevada), Kenneth Cole (U.S. Army Dugway Proving Grounds), Wayne Einfeld (SNL), Cheryl Estill (CDC), Misty Hein (CDC), Robert Knowlton (SNL), Paula Krauter (SNL), Laura Rose (CDC), and Nancy Valentine (PNNL). 



\section{Acronyms and Abbreviations}

$\begin{array}{ll}\text { \%RSD } & \text { percent relative standard deviation } \\ \text { BA } & \text { Bacillus anthracis } \\ \text { CDC } & \text { Centers for Disease Control and Prevention } \\ \text { CFU } & \text { colony forming unit } \\ \text { DHS } & \text { U.S. Department of Homeland Security } \\ \text { DoD } & \text { U.S. Department of Defense } \\ \text { DOE } & \text { U.S. Department of Energy } \\ \text { EPA } & \text { U.S. Environmental Protection Agency } \\ \text { FBI } & \text { Federal Bureau of Investigation } \\ \text { FNR } & \text { false negative rate } \\ \text { FPR } & \text { false positive rate } \\ \text { GAO } & \text { Government Accountability Office } \\ \text { LOD } & \text { limit of detection } \\ \text { NIST } & \text { National Institute of Standards and Technology } \\ \text { PNNL } & \text { Pacific Northwest National Laboratory } \\ \text { RE } & \text { recovery efficiency } \\ \text { SD } & \text { standard deviation } \\ \text { S\&T } & \text { Science and Technology Directorate } \\ \text { SNL } & \text { Sandia National Laboratories } \\ \text { U.S. } & \text { United States } \\ \text { VSP } & \text { Visual Sample Plan } \\ & \end{array}$





\section{Contents}

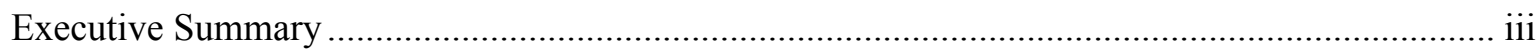

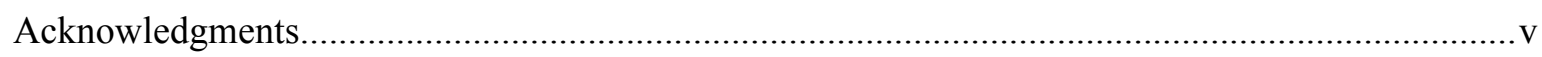

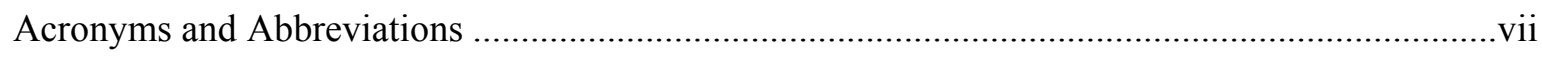

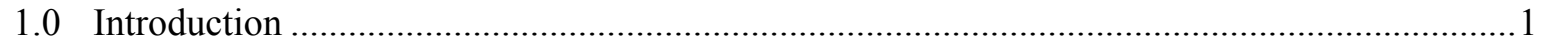

2.0 Motivation for Summarizing and Assessing the Gaps of Previous Anthrax Laboratory

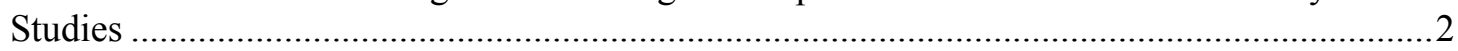

3.0 Summary of Anthrax-Related Laboratory Studies ............................................................

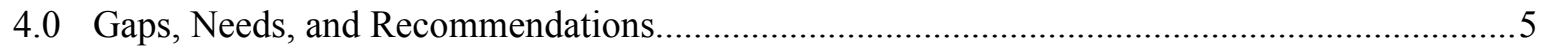

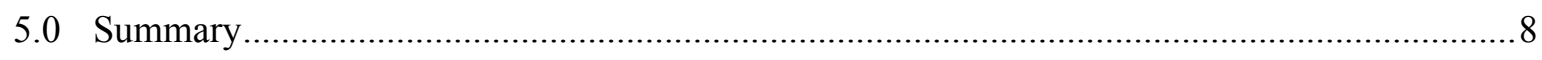

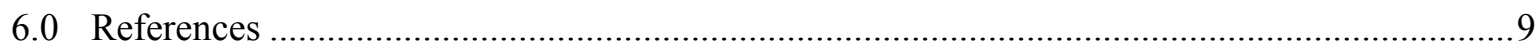

Appendix - Detailed Summary Tables of Test Conditions and Results from Laboratory Studies of Swab, Wipe, and Vacuum Sampling of Surfaces Contaminated with Bacillus anthracis Surrogates.

\section{Tables}

1 Descriptions of columns in tables summarizing the characteristics and results of laboratory studies on surface sampling of Bacillus anthracis and surrogates.......

2 Summary of test conditions for swab sampling studies ....................................................... 13

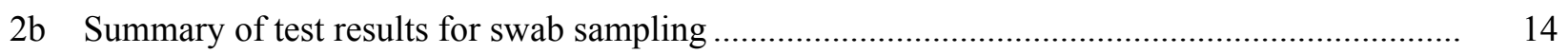

3a Summary of test conditions for wipe sampling studies ...................................................... 15

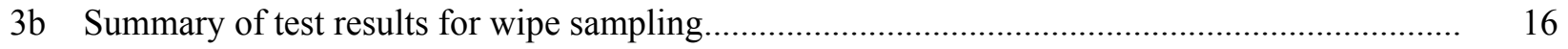

4a Summary of test conditions for vacuum sampling studies .................................................... 17

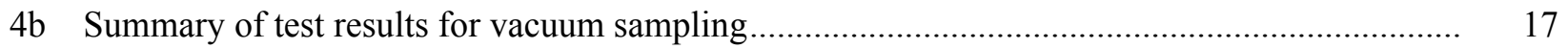

5a Summary of test conditions for storage and stability tests.................................................... 18

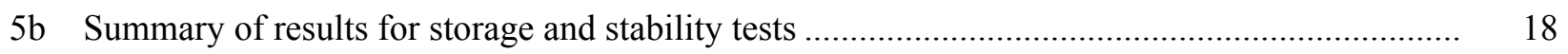

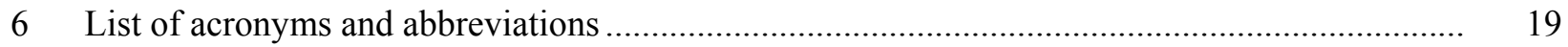

7 Numbers of the 20 surface sampling studies that investigated various factors and reported specific results 



\subsection{Introduction}

In 2001, letters containing Bacillus anthracis (BA) contaminated areas of the Hart Senate office building in Washington, DC and postal facilities that processed the letters. A congressional inquiry and Government Accountability Office (GAO) investigation (GAO 2005a, 2005b) identified two main concerns regarding the methods used to characterize and clear buildings. One main concern was the reliance on sampling specific locations where it was thought BA would be found. This type of sampling approach is referred to as judgmental (or targeted) sampling. The GAO reports identified the need to use statistical (or probabilistic) sampling so that when all results are negative, a building (or area within a building) can be cleared with a known level of statistical confidence. The second main concern was that methods used in the sampling and analysis process (i.e., sample collection, storage/transportation, processing/extraction, and analytical) were not validated. The lack of validated methods raised questions about the reliability of the negative sampling results obtained.

Several organizations are working to address the GAO concerns, including the U.S. Department of Homeland Security (DHS), U.S. Environmental Protection Agency (EPA), Centers for Disease Control and Prevention (CDC), National Institute of Standards and Technology (NIST), U.S. Department of Defense (DoD), Federal Bureau of Investigation (FBI), Pacific Northwest National Laboratory (PNNL), and Sandia National Laboratories (SNL). Some key activities to address the GAO's main concerns are:

A. developing a sampling-strategy document that describes appropriate uses of judgmental and statistical sampling approaches

B. validating methods associated with the sampling and analysis processes

C. developing formulas to estimate the BA contamination, quantify the bias and uncertainty of the estimate, calculate the confidence in characterization and clearance decisions, and calculate the number of samples necessary to achieve the desired uncertainty and confidence in such decisions.

For conciseness in subsequent discussions, these are referred to as Activities A, B, and C.

Activities $\mathrm{B}$ and $\mathrm{C}$ require quantitative information on the performance of sampling and analysis methods for indoor surface samples. Such information can be obtained from laboratory and field studies, although laboratory studies are best suited for certain performance measures because the level of contamination can be better controlled. Hence, we identified previous laboratory studies that were conducted to assess the performance of swab, wipe, and vacuum sample collection methods and subsequent sample storage/transportation, processing/extraction, and analysis methods for surfaces contaminated by BA or surrogates. The test conditions and results of the studies were summarized and assessed to determine whether there were any substantive gaps. The summary information, gaps and needs that were found, and recommendations made to address the gaps and needs, are presented and discussed in this report. Edmonds (2009) also reviewed the literature and discusses some needs and perspectives for future work. However, this report is more comprehensive and is based on tabular summaries of previous studies that make gaps and needs easier to identify.

The following section discusses how Activities B and C motivated the work discussed in this report. The two subsequent sections 1) identify the previous laboratory studies that were considered in this effort 
and summarize their characteristics and results, and 2) discuss the gaps and needs identified, and make several recommendations to address the gaps and needs. The final section summarizes the work.

\subsection{Motivation for Summarizing and Assessing the Gaps of Previous Anthrax Laboratory Studies}

Activities $\mathrm{A}$ and $\mathrm{C}$ involve developing sampling strategies, approaches, and formulas that will provide high confidence in the results of characterizing building contamination, and 2) clearing an uncontaminated or decontaminated building for reoccupation. Statistical and combined judgmental and random sampling (Sego et al. 2010) approaches provide for calculating the number of samples necessary to achieve the desired confidence for detecting contamination and clearing uncontaminated or decontaminated buildings. These approaches also provide for calculating the confidence in characterization or clearance decisions based on sampling results following contamination incidents. The Visual Sample Plan (VSP) software (VSP Development Team 2010) implements many approaches and methods for environmental sampling. Judgmental sampling provides for using the situational knowledge and experience of a field sampler in many situations where statistical samples may not be necessary to detect contamination.

Activity B involves validating methods in the sampling and analysis process for BA contamination in buildings. Validating a method consists of several actions (ISO/IEC 2005), two of which include 1) quantifying the uncertainty (repeatability and reproducibility) and accuracy of results obtained by the method, and 2) quantifying relevant performance metrics, such as recovery efficiency (RE), false negative rate (FNR), false positive rate (FPR), and limit of detection (LOD). Hence, it is important to understand how much of this type of information exists for the previous laboratory studies that investigated sampling of surfaces contaminated with BA or surrogates. This report summarizes estimates of the above performance metrics and corresponding uncertainties reported in the references that document the studies.

The FNR plays an important role in Activity C, because a higher FNR will reduce the confidence in contamination detection and clearance decisions, which can be offset by taking more samples. Further, the overall RE of a sampling process contributes to determining the overall FNR. Here "overall" means over all steps in the sampling process: sample collection, storage/transportation, processing/extraction, and analysis (e.g., culturing and counting). Having sufficient experimental data to determine the overall RE and FNR (and their uncertainties) for swab, wipe, and vacuum samples on various surfaces at various levels of contamination (and any other influencing factors), is important in assessing risks (of failing to detect spores or erroneously clearing an area) and in developing sampling strategies and approaches.

Standard statistical formulas assume that the overall FNR $=0$ when calculating 1) the number of samples required to achieve the desired confidence for a characterization or clearance sampling goal, and

2) the uncertainty and confidence associated with a characterization or clearance decision using a specific sampling approach implemented following a contamination incident. When FNR $=0$, the formulas account only for the uncertainty in results associated with the specific type of statistical or hybrid sampling approach being used. However, the overall FNR is affected by anything in the sampling process that might yield a false negative, including (i) the RE of a sampling method (e.g., swab, wipe, or vacuum), (ii) the RE of storage or transportation steps, if applicable, (iii) the RE of the processing/ extraction step (i.e., extracting the contaminant from the sample medium), and (iv) the uncertainty of the 
analytical/detection method and equipment. Hence, it is important in laboratory studies to quantify the REs and uncertainties affecting the results of a method at each step in the process, so that the overall RE and overall FNR can be determined.

Standard statistical formulas for calculating 1) and 2) in the previous paragraph can be extended to address situations in which the overall FNR $>0$. The VSP software (VSP Development Team 2010) generally implements standard formulas to calculate the numbers of samples to address situations in which the overall FNR $=0$. Extended formulas, applicable when the overall FNR $>0$, have only been developed for a statistical, grid-sampling approach for detecting contaminated areas of specified size (socalled hotspot sampling). PNNL plans to develop extended formulas for calculations 1) and 2) when FNR $>0$ with other statistical sampling approaches recommended in a multiagency sampling strategy (not yet completed). Adequate information on the FNR performance of sampling and analysis methods is needed as input to this work.

Previous study data indicates the overall RE and FNR may depend on the surface concentration of BA contamination, how the contamination is deposited on a surface, the surface material, the specifics of a sampling method, and possibly other factors. Hence, laboratory studies should investigate the performance of the sampling and analysis process for the most commonly used sampling methods over a range of BA (or surrogate) surface concentrations, different deposition methods, different surface materials, etc. In fact, several such studies have been performed, but important questions are whether the studies adequately 1) cover the combinations of factors that may affect results, and 2) quantify performance measures of the methods and conditions studied.

\subsection{Summary of Anthrax-Related Laboratory Studies}

Previous laboratory studies were identified that used 1) swab, wipe, and vacuum sampling methods to collect BA (or surrogate) contamination from various porous and nonporous surfaces, and 2) culture and count methods to quantify the contamination. Only BA/surrogate contamination and count methods were considered, to keep the scope of the effort manageable. We focused on laboratory studies (as opposed to field studies or data from BA contamination incidents) because they provide a basis for quantifying actual contamination levels, and using those values to estimate REs and other method performance metrics such as FNR. The 20 studies included in our summary and gap assessment are: Buttner et al. (2001, 2004a, 2004b), Rose et al. (2004), Hodges et al. (2006), Nellen et al. (2006), Brown et al. (2007a, 2007b, 2007c), Quizon et al. (2007), Almeida et al. (2008), Frawley et al. (2008), Montgomery and Camp (2008), Valentine et al. (2008), Edmonds et al. (2009), Estill et al. (2009), Hodges et al. (2010), Einfeld et al. (2011), Krauter et al. (2011), and Rose et al. (2011).

The characteristics and results of relevant tests in each study are summarized in four tables, each with "a" and "b" parts. The "a" part of each table summarizes the characteristics of the studies, while the "b" part summarizes the results of the studies. The " $b$ " parts of tables are further divided into three categories of information: 1) Recovery concentration results - Mean \& \%RSDs, 2) Recovery efficiency (RE) Mean \& \%RSDs, and 3) LOD, FNR and FPR. All percent relative standard deviation (\%RSD) values are rounded to zero decimal places. Table 1 describes the study characteristics and results in the columns of the summary tables. In each table, different studies are represented by groups of rows, in which the rows correspond to the tests performed within a study. Because the four tables that summarize groups of tests 
and results are long, they are included in the Appendix as Tables A.1 (swab sampling), A.2 (wipe sampling), A.3 (vacuum sampling), and A.4 (one storage/transportation study that did not involve surface sampling). Condensed summaries of Tables A.1 to A.4 are presented in Tables 2 to 5 with "a" and "b" parts as described previously. Acronyms and abbreviations used in Tables 2 to 5 and Tables A.1 to A.4 are defined in Table 6.

In reading the publications that document the studies summarized in this report, we noticed many differences in the way tests and calculations were performed and reported. The main differences are documented in Tables 2 to 5 (and Tables A.1 to A.4), but some differences were too detailed to document. A common difference was in the units used to report results. Whenever possible, we performed calculations using information in the publications to convert results reported in a given set of units to the common set of units used in the "b" parts of Tables 2 to 5 (and Tables A.1 to A.4). There were also experimental and calculational differences in the ways studies used positive controls to obtain the "actual" contamination values used as the denominator in calculating RE. These differences affect the RE values and their uncertainties, in some cases potentially making the uncertainties larger than they needed to be.

Table 7 documents key study characteristics and results from Tables 2 to 5 (and Tables A.1 to A.4). Specifically, it summarizes the numbers of the 20 laboratory studies that varied several study factors and reported various performance metrics. Table 7 shows that 13, 12, and 5 studies investigated swab, wipe, and vacuum sampling, respectively. Some studies investigated more than one type of sampling. Only Almeida et al. (2008) investigated storage/transportation effects on sampling results. An additional storage/transportation study has been completed, but the results have only been partially released (O'Connell et al. 2010, Perry et al. 2010). The information in those references was not complete enough to include the study in this report.

The "zero" entries in Table 7 denote combinations of study characteristics/results that were not addressed by the 20 studies. Table 7 shows that few of the 20 studies had results available from varying the factors: 1) agent (contaminant), 2) agent deposition method, 3) sample-collection medium, 4) wetting agent for the sampling medium (swab and wipe), 5) storage/transport conditions, and 6) processing/extraction method. Lab-to-lab uncertainties of REs were reported only by Estill et al. (2009) for swabs, wipes, and vacuum samples; Hodges et al. (2010) for swab samples; and Rose et al. (2011) for wipe samples. Run-to-run uncertainties were quantified by Estill et al. (2009) for swab, wipe, and vacuum samples; Edmonds et al. (2009) and Hodges et al. (2006) for swab samples; and Montgomery and Camp (2008) for vacuum samples. Even when uncertainties reported in some studies include lab-to-lab and/or run-to-run uncertainties, the numbers of labs and runs were typically small, indicating these sources of uncertainty are poorly estimated. Lab-to-lab and run-to-run uncertainties are expected to be substantial contributors to uncertainty and are missing from most of the studies. Hence, the uncertainties reported by many studies can be expected to underestimate the total uncertainties in surface sampling results. Also, the uncertainties may be underestimated because of smaller uncertainties when applying sampling and analysis methods in controlled laboratory environments than may occur in actual contamination incidents.

LOD and FNR/FPR results are rarely reported in the laboratory study results, as shown in Table 7. As discussed previously, the FNR and RE (each of which may be a function of contaminant concentration and other factors) play a key role in the framework for determining the number of samples necessary to achieve a characterization or clearance goal with a specified confidence. Hence, it is important to experimentally quantify how RE and FNR depend on contaminant surface concentration and other 
sampling and environmental factors. Table 7 shows that only a few of the 20 studies varied the contaminant at different surface concentrations. Most of these studies investigated three or fewer concentrations, although Hodges et al. (2006) investigated six and Krauter et al. (2011) investigated eight. Montgomery and Camp (2008) [vacuum results], Estill et al. (2009), Hodges et al. (2010), Rose et al. (2011), and Krauter et al. (2011) found that RE did not depend on surface concentration for the range of concentrations tested. However, Hodges et al. (2006) and Edmonds et al. (2009) reported that RE increased as concentration increased. Of the studies that investigated different surface concentrations, only Krauter et al. (2011) presented equations for FNR as a function of surface concentration (for each of six surface materials). Estill et al. (2009) mentioned using probit regression to develop a probability-ofdetection (equivalent to FNR) curve as a function of contaminant concentration, but did not present the results. The general lack of FNR data (and of FNR and RE curves as functions of surface concentration, surface material, and other influencing factors) is a significant gap in all studies except Krauter et al. (2011).

The studies summarized in Tables 2 to 5 have large ranges of mean RE values and sample-within-run uncertainty $\left(\% \mathrm{RSD}_{\mathrm{RE}}\right)$ values. The following ranges of $\mathrm{RE}$ and $\% \mathrm{RSD}_{\mathrm{RE}}$ exclude data from directinoculation tests in which surfaces were not sampled with a swab, wipe, or vacuum. For swabs, RE ranged from $0-92.7 \%$ and $\% \mathrm{RSD}_{\mathrm{RE}}$ ranged from 6-550. For wipes, RE ranged from $1-97 \%$ (excluding a questionable $120 \%$ value in the Estill et al. 2009 study) and $\% \mathrm{RSD}_{\mathrm{RE}}$ ranged from $0-316$. For vacuums, $\mathrm{RE}$ ranged from $0.02-36 \%$ and $\% \mathrm{RSD}_{\mathrm{RE}}$ ranged from $10-130$. These ranges of $\% \mathrm{RSD}_{\mathrm{RE}}$ values represent the uncertainty in RE results from replicate tests performed at the same time, generally by the same samplers and the same laboratory personnel (because that is what most studies reported). Hence, these $\% \mathrm{RSD}_{\mathrm{RE}}$ ranges do not include all relevant sources of variation, and can be expected to underestimate the total uncertainty.

The wide ranges of RE values summarized above are a result of the effects of several factors varied within and across the studies (e.g., contaminant surface concentration; contaminant deposition method; surface material being sampled; materials and specifics of swabs, wipes, and vacuum socks; wetting agent for swabs and wipes; specifics of sample preparation and extraction methods, and counting method) as shown in Tables $2 \mathrm{a}-5 \mathrm{a}$. Krauter et al. (2011) also indicate that FNR values can be affected by such factors. Hence, the dependence of RE, FNR, LOD, and their total uncertainties (including all sources of uncertainty in the sampling and analysis process) on such factors needs to be quantified. Ideally, all data from each of the swab, wipe, and vacuum studies would be combined and a statistical analysis performed to estimate the effects of the quantitative and qualitative test factors on RE, FNR, and LOD. However, FNR and LOD were not reported in enough studies to do this. Even for the widely reported RE, the number of test factors is large with test factors sometimes having many possible values/options. Also, there may have been interactive effects on performance metrics between some test factors in the studies. Although it was beyond the scope of this work to attempt such a combined-data statistical analysis, our initial assessment is that there are not enough data for this exercise to be successful given the large number of factor combinations and possible interactions in the swab, wipe, and vacuum studies.

\subsection{Gaps, Needs, and Recommendations}

This section discusses several gaps and needs identified based on the summaries of laboratory studies of swab, wipe, and vacuum sampling and analysis methods in Tables 2 to 5 (and Tables A.1 to A.4). 
Recommendations are made for additional laboratory studies and other evaluations to address these gaps and needs. In what follows, "performance results" refers to RE, FNR, LOD, and their uncertainties, considering uncertainty contributions from all steps of the sampling and analysis process (i.e., sample collection, storage/transportation, processing/extraction, and analytical methods).

Realistic versus Laboratory Conditions: Price et al. (2009) emphasize the importance of quantifying the performance of methods (sample collection, storage/transportation, processing/extraction, and analysis) under realistic conditions and not just highly sanitized laboratory conditions. Some studies investigated other material added with the BA or surrogate spores: non-BA organism (Buttner et al. 2001), grime (Einfeld et al. 2011), and silicon dioxide (Brown et al. 2007a, 2007b, 2007c; Estill et al. 2009). Buttner et al. (2001) and Einfeld et al. (2011) found that material added to the BA or surrogate spores can have a significant effect on RE. Data from field studies (e.g., Amidan et al. 2007, Piepel et al. 2009) and the 2001 BA letter incident that involve realistic conditions are available, but don't provide for accurately assessing RE and FNR in the way that laboratory studies do. More work may be needed to quantify the effects on method performance results of surfaces being contaminated with other biological organisms and material in addition to BA. Ideally such studies should be performed with three levels of each other organism/material (none, low, high) to better assess the effects of such factors on sampling performance results.

Bacillus anthracis (BA) versus Surrogates: Previous laboratory studies have used BA and several surrogates (see Tables 2 to 5). However, these studies have not quantitatively addressed the relationship between performance results of swab, wipe, and vacuum methods with BA and with its surrogates. If it is not feasible to use BA and key surrogates in a new laboratory study to establish these relationships, then measurements of organism properties relevant to sampling and resuspension studies should be performed on BA and the various surrogates. The data should then be used to establish the relationships for sampling and analysis performance results between BA and its surrogates.

Deposition Method: Previous laboratory studies generally used one of two contaminant deposition methods: 1) contaminant in liquid (water, ethanol, or a mixture of the two) that was allowed to dry after deposition by wet aerosol or liquid drops, and 2) dry aerosol. Only one swab study (Edmonds et al. 2009) compared REs for different deposition methods. No wipe or vacuum studies varied the deposition method. Edmonds et al. (2009) found that for high contaminant concentrations, REs differed significantly depending on liquid vs. dry deposition, with the difference depending on the swab sampling method. More work is needed to quantify the difference in performance results of swab, wipe, and vacuum sampling and analysis methods as a function of the contaminant deposition method, as well as functions of other influencing factors (e.g., contaminant concentration).

Surface Types: A wide variety of surface materials were investigated in the swab, wipe, and vacuum sampling studies summarized in Tables 2 to 4 . However, some studies investigated only stainless steel or a limited number of surface materials. Method performance results were generally reported separately for each surface material, without any attempt to develop relationships between performance results and characteristics of the surface materials. The exception was Krauter et al. (2011), who compared mean REs, FNR, and LOD to the roughness index of the surfaces investigated. More work is needed to develop equations that relate method performance to one or more surface-characteristic variables (as well as any other influencing factors). Such equations can then be used to estimate method performance for various surface materials. 
Storage and Transportation of Samples: Only two previous studies have investigated the effects of storage and/or transportation conditions on method performance results. Almeida et al. (2008) investigated the effects of different additives, storage times, and storage temperatures on the stability of BA in water. However, that work is not directly relevant to surface sampling, because it would be swab, wipe, and vacuum samples that would be stored and/or transported to laboratories (which would then perform the processing/extraction and analysis steps). A second study investigated the effects of storage and transportation factors on swab samples, but only partial results have been released (O'Connell et al. 2010, Perry et al. 2010). Because this study only addresses swab samples, additional studies or evaluations are needed to quantify how sampling and analysis methods for wipe and vacuum samples are affected by storage and transportation methods.

Recovery Efficiency of Sample Collection vs. Processing: Several studies investigated the contributions of sample collection and sample processing/extraction to the overall RE of a method. For directly inoculated petri dishes, Buttner et al. (2001) reported sample collection efficiency, processing efficiency, and overall RE for two swab and one wipe sampling methods. For all three, the majority of the inefficiency came in the processing step, not the sample collection step. Results from other studies that investigated and reported REs for sample collection and processing steps were similar in some cases (Rose et al. 2004), while in other cases the sample collection inefficiency was similar to or larger than processing inefficiency (Buttner et al. 2001; Nellen et al. 2006; and Brown et al. 2007a, 2007b). In all cases, processing/extraction inefficiency is large enough that optimizing the specifics of the processing/extraction step would be very important in maximizing RE. The optimal processing conditions may depend on the sample collection material, the extraction solution, and the methods of dissociation (e.g., vortexing, sonication).

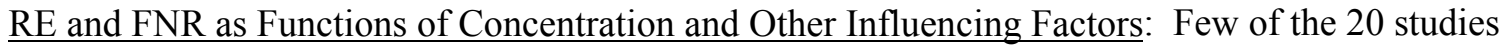
reported FNR data and only Krauter et al. (2011) developed relationships between low contaminant concentrations and the FNR performance (for the sponge-stick wipe method). Similar work is needed for other surface sampling and analysis methods that may be used when BA surface concentrations are low. Quantifying the FNR-concentration relationships is critical because FNR $>0$ significantly affects 1) the number of samples required to achieve desired confidences in characterization and clearance decisions, and 2) the confidence in characterization and clearance decisions made using sample and analysis data following a contamination incident. On the other hand, if surface concentrations are at levels much higher than when false negatives begin to appear, then the magnitude of RE and its dependence on surface concentration are not very important if the goal is to merely detect contamination (versus to accurately quantify the amount/concentration of contaminant).

Estimates of Main Sources of Uncertainty: Previous laboratory studies did not capture the main sources of uncertainty affecting performance results. Many of the studies investigated only short-term, within-run uncertainties (repeatability) and did not investigate run-to-run or lab-to-lab uncertainties (reproducibility). Hence, the estimates of uncertainty in performance measures for most of the studies in Tables 2-5 (and Tables A.1-A.4) can be expected to underestimate the total uncertainty. Further, in some studies that captured more than one source of uncertainty, the statistical measures of uncertainty reported may have been improperly calculated. A common error is to calculate the standard deviation (or \%RSD) from a set of data subject to more than one source of uncertainty when the data provides for separately estimating the uncertainties. The correct approach is to use statistical variance-component estimation to separately estimate the standard deviation (or $\% \mathrm{RSD}$ ) for each source of uncertainty, and then properly combine the separate estimates into an estimate of total uncertainty. The studies that best met the goals of 
quantifying repeatability and reproducibility uncertainties are the swab and wipe validation studies (Hodges et al. 2010 and Rose et al. 2011, respectively). If additional studies of this type are needed, they should be designed to capture repeatability and reproducibility uncertainties in the steps of the sampling and analysis process (i.e., sample collection, storage/transportation, processing/extraction, and analytical).

Control Samples: Control samples (e.g., positive, negative, sample-medium blanks, laboratory blanks) are necessary to quantify performance measures such as RE, FNR, and FPR. Although the studies summarized in this report included control samples, FNR and FPR were seldom reported. Positive controls for deposition load used to calculate RE should be co-located with each surface sample, although this was not done for some of the studies. That way, the RE for each surface sample can be calculated using the co-located positive control sample, if necessary. This approach corrects for unavoidable systematic variation in contaminant deposition in a chamber or controlled test area, so that such differences do not inflate the uncertainties of RE.

We recommend that additional experimental studies and non-experimental evaluations be performed to address the above gaps and needs. Additional experimental studies should be performed selectively, along with non-experimental evaluations, so that the combination of previous and new results provide for adequately quantifying the performance characteristics (e.g., RE, FNR, LOD, and uncertainties) of swab, wipe, and vacuum sampling methods, accounting for all the steps of the sampling and analysis process.

\subsection{Summary}

The GAO reports (GAO 2005a, 2005b) recommended the use of statistical sampling approaches to support making high-confidence characterization and clearance decisions for biological contamination incidents. Methodology and related data for quantifying the overall uncertainty and confidence associated with characterization and clearance decisions is needed to address the GAO concerns. This methodology must account for the performance and uncertainties of relevant statistical sampling approaches, sample collection methods, sample transportation and/or storage effects, sample processing and extraction methods, and sample analysis methods. PNNL plans to develop methodology and formulas that account for FNR $>0$ when calculating 1) the number of samples needed with a given statistical sampling approach to achieve specified confidence in characterization and clearance decisions, and 2) the confidence in characterization and clearance decisions using statistical sampling data obtained following a contamination incident. For both of these, method performance data are needed to provide input values for the formulas.

The literature summarized and discussed in this report contains considerable data on the performance of swab, wipe, and vacuum surface-sampling methods for BA (or surrogates) contamination. However, that body of work does not provide for fully quantifying the performance of swab, wipe, and vacuum sampling methods while accounting for all steps of the sampling process (sample collection, transportation/storage, processing/extraction, and analytical). Additional work is needed to quantify the biases (e.g., REs), FNRs, and uncertainties associated with the steps of the sampling process over a range of contamination concentrations for relevant surface materials that are sampled/extracted/analyzed by relevant methods. Further, most of the studies summarized in this report did not capture one or more applicable sources of uncertainty. Hence, data from those studies can be expected to underestimate the overall uncertainties in the sampling process. In summary, to adequately quantify all of the sources of 
bias and uncertainty in the steps of the sampling process (collection, storage/transportation, processing/extraction, and analysis), additional laboratory studies and other evaluations should be conducted to augment the data from previous studies.

\subsection{References}

Almeida, J.L., Harper, B. and Cole, K.D. (2008) Bacillus anthracis spore suspensions: determination of stability and comparison of enumeration techniques. J Appl Microbiol 104, 1442-1448.

Amidan, B.G., Piepel, G.F., Matzke, B.D., Filliben, J.J. and Jones, B.J. (2007) Experimental Design for the INL Sample Collection Operational Test, PNNL-17129, Pacific Northwest National Laboratory, Richland, WA. http://www.pnnl.gov/main/publications/external/technical_reports/PNNL-17129.pdf

Brown, G.S., Betty, R.G., Brockmann, J.E., Lucero, D.A., Souza, C.A., Walsh, K.S., Boucher, R.M., Tezak, M., Wilson, M.C. and Rudolph, T. (2007a) Evaluation of a wipe surface sample method for collection of Bacillus spores from nonporous surfaces. Appl Environ Microbiol 73, 706-710.

Brown, G.S., Betty, R.G., Brockmann, J.E., Lucero, D.A., Souza, C.A., Walsh, K.S., Boucher, R.M., Tezak, M.S., Wilson, M.C., Rudolph, T., Lindquist, H.D. and Martinez, K.F. (2007b) Evaluation of rayon swab surface sample collection method for Bacillus spores from nonporous surfaces. $J$ Appl Microbiol 103, 1074-1080.

Brown, G.S., Betty, R.G., Brockmann, J.E., Lucero, D.A., Souza, C.A., Walsh, K.S., Boucher, R.M., Tezak, M.S. and Wilson, M.C. (2007c) Evaluation of vacuum filter sock surface sample collection method for Bacillus spores from porous and non-porous surfaces. J Environ Monitor 9, 666-671.

Buttner, M.P., Cruz-Perez, P. and Stetzenbach, L.D. (2001) Enhanced detection of surface-associated bacteria in indoor environments by quantitative PCR. Appl Environ Microbiol 67, 2564-2570.

Buttner, M. P., Cruz, P., Stetzenbach, L.D., Klima-Comba, A.K., Stevens, V.L. and Cronin, T.D. (2004a) Determination of the efficacy of two building decontamination strategies by surface sampling with culture and quantitative PCR analysis. Appl Environ Microbiol 70, 4740-4747.

Buttner, M.P., Cruz, P., Stetzenbach, L.D., Klima-Comba, A.K., Stevens, V.L. and Emanuel, P.A. (2004b) Evaluation of the biological sampling kit (BiSKit) for large-area surface sampling. Appl Environ Microbiol 70, 7040-7045.

Edmonds, J.M. (2009) Efficient methods for large-area surface sampling of sites contaminated with pathogenic microorganisms and other hazardous agents: current state, needs, and perspectives. Appl Microbiol Biotech 84, 811-816.

Edmonds, J.M., Collett, P.J., Valdes, E.R., Skowronski, E.W., Pellar, G.J. and Emanuel, P.A. (2009) Surface sampling of spores in dry-deposition aerosols. Appl Environ Microbiol 75, 39-44. 
Einfeld, W., Boucher, R.M., Tezak, M.S., Wilson, M.C. and Brown, G.S. (2011) Evaluation of Surface Sampling Method Performance for Bacillus Spores on Clean and Dirty Outdoor Surfaces, SAND20114085, Sandia National Laboratories, Albuquerque, NM.

Estill, C.F., Baron, P.A. Beard, J.K., Hein, M.J., Larsen, L.D., Rose, L., Schaefer III, F.W., Noble-Wang, J., Hodges, L., Lindquist, H.D.A., Deye, G.J. and Arduino, M.J. (2009) Recovery efficiency and limit of detection of aerosolized Bacillus anthracis Sterne from environmental surface samples. Appl Environ Microbiol 75, 4297-4306.

Frawley, D.A., Samaan, M.N., Bull, R.L., Robertson, J.M., Mateczun, A.J. and Turnbull, P.C.B. (2008) Recovery efficiencies of anthrax spores and ricin from nonporous or nonabsorbent and porous or absorbent surfaces by a variety of sampling methods. J Forensic Sciences 53, 1102-1107.

Government Accountability Office (GAO). (2005a) Anthrax Detection: Agencies Need to Validate Sampling Activities in Order to Increase Confidence in Negative Results (Report to the Chairman, Subcommittee on National Security, Emerging Threats, and International Relations, House Committee on Government Reform, House of Representatives), GAO-05-251, U.S. Government Accountability Office, Washington, DC.

Government Accountability Office (GAO). (2005b) Anthrax Detection: Agencies Need to Validate Sampling Activities in Order to Increase Confidence in Negative Results, (Testimony before the Chairman, Subcommittee on National Security, Emerging Threats, and International Relations, House Committee on Government Reform, House of Representatives), GAO-05-493T, U.S. Government Accountability Office, Washington, DC.

Hodges, L.R., Rose, L.J., Peterson, A., Noble-Wang, A.J. and Arduino, M.J. (2006) Evaluation of a macrofoam swab protocol for the recovery of Bacillus anthracis spores from a steel surface. Appl Environ Microbiol 72, 4429-30.

Hodges, L.R., Rose, L.J., O'Connell, H. and Arduino, M.J. (2010) National validation study of a swab protocol for the recovery of Bacillus anthracis spores from surfaces. J Microbiol Meth 81, 141-146.

ISO/IEC (2005) Validation of Methods, Section 5.4.5, in International Standard 17025: General Requirements for the Competence of Testing and Calibration Laboratories, Second Edition, ISO/IEC 17025:2005(E), Geneva, Switzerland.

Krauter, P.A., Piepel, G.F., Boucher, R., Tezak, M., Amidan, B.G. and Einfeld, W. (2011) False Negative Rate and Other Performance Measures of a Sponge-Wipe Surface Sampling Method for Low Contaminant Concentrations, SAND2011-3395, Sandia National Laboratories, Albuquerque, NM.

Montgomery, N.K. and Camp, D.W. (2008) The Efficiency of Collecting Bacillus anthracis Spores from HVAC Filters and Carpet by Vacuum, Wipe, and Bulk Sampling Methods, LLNL-PRES-408494.

Lawrence Livermore National Laboratory, Livermore, CA.

Nellen, J., Rettberg, P., Horneck, G. and Streit, W.R. (2006) Planetary protection - approaching uncultivable microorganisms. Adv Space Res 38, 1266-1270. 
O'Connell, H., Rose, L., Perry, A., Noble-Wang, J. and Arduino, M. (2010) Shipping integrity of Bacillus anthracis swab samples, poster presented at the American Society for Microbiology Biodefense Conference, New Orleans, LA, Feb. 2010, Centers for Disease Control and Prevention, Atlanta, GA.

Perry, A., O’Connell, H., Rose, L. and Noble-Wang, J. (2010) Storage effects on sample integrity of environmental surface sampling specimens with Bacillus anthracis, primary and secondary containment, Poster presented at the American Society for Microbiology Biodefense Conference, New Orleans, LA, Feb. 2010, Centers for Disease Control and Prevention, Atlanta, GA.

Piepel, G.F., Amidan, B.G., and Matzke, B.D. (2009) Experimental and Sampling Design for the INL-2 Sample Collection Operational Test, PNNL-18187, Pacific Northwest National Laboratory, Richland, WA. http://www.pnnl.gov/main/publications/external/technical_reports/PNNL-18187.pdf

Price, P.N., Sohn, M.D., Lacommare, K.S.H. and McWilliams, J.A. (2009) Framework for evaluating anthrax risk in buildings. Environ Sci Technol 43, 1783-1787.

Quizon, R., Quizon, J., Proescher, A., Bare, C., Goodenow, B., Wagner, M. and Van Gieson, E. (2007) Test and Evaluation of Surface Sampling Approaches Before and After Small-Scale Fumigation-Based Decontamination Events, NSTD-07-0592. Applied Physics Laboratory, National Security Technology Department, John Hopkins University, Laurel, MD.

Rose, L., Jensen, B., Peterson, A., Banerjee, S.N. and Arduino, M.J. (2004) Swab materials and Bacillus anthracis spore recovery from nonporous surfaces. Emerg Infect Dis 10, 1023-1029.

Rose, L.J., Hodges, L., O'Connell, H. and Noble-Wang, J. (2011) National validation study of a cellulose sponge-wipe processing method for use after sampling Bacillus anthracis spores from surfaces. Appl Environ Microbiol, doi:10.1128/AEM.05377-11.

Valentine, N.B., Butcher, M.G., Su, Y.F., Jarman, K.H., Matzke, M., Webb-Robertson, B.J., Panisko, E.A., Seiders, B.A.B. and Wahl, KL. (2008) Evaluation of sampling tools for environmental sampling of bacterial endospores from porous and nonporous surfaces. $J$ Appl Microbiol 105, 1107-1113.

VSP Development Team (2010) Visual Sample Plan: A Tool for Design and Analysis of Environmental Sampling. Version 6.0. Pacific Northwest National Laboratory, Richland, WA. http://vsp.pnl.gov. 
Table 1. Descriptions of columns in tables summarizing the characteristics and results of laboratory studies on surface sampling of Bacillus anthracis and surrogates.

\begin{tabular}{|c|c|}
\hline \multicolumn{2}{|c|}{ Study Characteristics (in Tables 2a, 3a, 4a, and 5a) } \\
\hline Reference & Author (Year) citation of publication that documents the study \\
\hline Test \# & $\begin{array}{l}\text { A number of the form } \mathrm{x} . \mathrm{y} \text { where } \mathrm{x} \text { is } 1,2,3, \ldots \text { for each study, and } \mathrm{y}=1,2,3 \text {, } \\
\ldots \text { for the tests within a study }\end{array}$ \\
\hline Agent & B. anthracis or related surrogate \\
\hline Agent deposition & Method used to deposit agent on test material \\
\hline Agent concentration & Concentration or amount of agent on surface \\
\hline Swab/Wipe/Vacuum type & Type or material of sample collection medium \\
\hline Wetting agent & Swab or Wipe: Liquid, if any, used to wet or premoisten the sampling material \\
\hline Vacuum technique & Vacuum: Technique in vacuuming (e.g., pattern, pressure applied) \\
\hline Relative humidity & Percent relative humidity in chamber or laboratory during testing \\
\hline Surface type & Material type of surface sampled \\
\hline Surface area sampled & Area of surface sampled \\
\hline Extraction liquid & Liquid used to extract spores from the sample \\
\hline Extraction method & Method used to prepare a sample and extract the contaminant \\
\hline Culture method/medium & Method/medium used to culture samples \\
\hline \# labs & The number of labs that participated in a study \\
\hline \# test runs & The number of test runs (set up and performed separately at different times) \\
\hline Total \# test samples & Total number of samples tested (i.e., over labs, runs, and samples within runs) \\
\hline \multicolumn{2}{|c|}{ Study Results (in Tables $2 \mathrm{~b}, 3 \mathrm{~b}, \mathbf{4 b}$, and $5 \mathrm{~b}$ ) } \\
\hline \multicolumn{2}{|c|}{$\begin{array}{ll}\text { Recovery Concentration Results - Mean \& \%RSDs } \\
\end{array}$} \\
\hline Reference & Same as for "Study characteristics" tables (see above) \\
\hline Test \# & Same as for "Study characteristics" tables (see above) \\
\hline Mean $\left(\mathrm{CFU} / \mathrm{cm}^{2}\right)$ & Mean surface concentration recovered \\
\hline Lab \%RSD & $\begin{array}{l}\text { Lab-to-lab percent relative standard deviation, which includes the variation from } \\
\text { preparing the samples, extraction, and analysis. }\end{array}$ \\
\hline Run \%RSD & $\begin{array}{l}\text { Percent relative standard deviation from replicate runs of a test performed at } \\
\text { different times. }\end{array}$ \\
\hline Sample-within-run \%RSD & $\begin{array}{l}\text { Percent relative standard deviation from replicate tests performed at the same } \\
\text { time (in one run) }\end{array}$ \\
\hline Total \%RSD & $\begin{array}{l}\text { Total percent relative standard deviation (including Lab, Run, and Sample- } \\
\text { within-run) }\end{array}$ \\
\hline \multicolumn{2}{|r|}{ Recovery Efficiency (RE) - Mean \& \%RSDs } \\
\hline RE mean $(\%)$ & Mean recovery efficiency \\
\hline RE lab \%RSD & Lab-to-lab percent relative standard deviation of RE \\
\hline RE run \%RSD & Run percent relative standard deviation of RE \\
\hline RE sample-within-run \%RSD & Sample-within-run percent relative standard deviation of RE \\
\hline RE total \%RSD & $\begin{array}{l}\text { Total percent relative standard deviation of RE (including Lab, Run, and } \\
\text { Sample-within-run) }\end{array}$ \\
\hline \multicolumn{2}{|r|}{ LOD, FNR, and FPR } \\
\hline Positive result & How a positive result (detection) was defined (e.g., CFU $\geq 1$ ) \\
\hline LOD definition & How the limit of detection (LOD) is defined \\
\hline LOD & Value of the limit of detection \\
\hline LOD SD or $95 \% \mathrm{CI}\left(\mathrm{CFU} / \mathrm{cm}^{2}\right)$ & Standard deviation or $95 \%$ confidence interval for the LOD \\
\hline FNR & $\begin{array}{l}\text { False negative rate (FNR) based on controlled tests where the sampled surface } \\
\text { was known to be contaminated but yielded a negative result }\end{array}$ \\
\hline FPR & $\begin{array}{l}\text { False positive rate (FPR) based on controlled tests where the sampled surface } \\
\text { was known to be uncontaminated but yielded a positive result }\end{array}$ \\
\hline
\end{tabular}


Table 2a. Summary of test conditions for swab sampling studies. Acronyms and abbreviations are defined in Table 6 and the footnotes.

\begin{tabular}{|c|c|c|c|c|c|c|c|c|c|c|c|c|c|c|c|}
\hline \multirow{2}{*}{$\begin{array}{l}\text { Reference } \\
\text { (lead author } \\
\text { and year) }\end{array}$} & \multirow[b]{2}{*}{$\begin{array}{c}\# \\
\text { tests }\end{array}$} & \multirow[b]{2}{*}{ Agent } & \multirow{2}{*}{$\begin{array}{l}\text { Agent } \\
\text { depo- } \\
\text { sition } \\
\end{array}$} & \multicolumn{2}{|r|}{$\begin{array}{c}\text { Agent } \\
\text { concentration(s) }\end{array}$} & \multirow[b]{2}{*}{ Swab types } & \multirow[b]{2}{*}{$\begin{array}{l}\text { Wetting } \\
\text { agent }\end{array}$} & \multirow[b]{2}{*}{ Surface types } & \multirow{2}{*}{$\begin{array}{l}\text { Surface } \\
\text { area } \\
\text { sampled } \\
\left(\mathrm{cm}^{2}\right) \\
\end{array}$} & \multirow{2}{*}{$\begin{array}{c}\text { Extract } \\
\text {-ion } \\
\text { liquid }\end{array}$} & \multirow{2}{*}{$\begin{array}{c}\text { Extraction } \\
\text { method }\end{array}$} & \multirow{2}{*}{$\begin{array}{l}\text { Culture } \\
\text { method, } \\
\text { medium }\end{array}$} & \multirow[b]{2}{*}{$\begin{array}{c}\# \\
\text { Labs }\end{array}$} & \multirow{2}{*}{$\begin{array}{c}\# \\
\text { test } \\
\text { runs }\end{array}$} & \multirow{2}{*}{$\begin{array}{c}\# \\
\text { samples } \\
\text { /test }\end{array}$} \\
\hline & & & & \# & $\begin{array}{c}\text { Range } \\
\left(\mathrm{CFU} / \mathrm{cm}^{2}\right)\end{array}$ & & & & & & & & & & \\
\hline Estill 2009 & 6 & BA-S & DA & 3 & $0.03-2$ & MF & BBT & $\mathrm{SS}, \mathrm{Cpt}$ & 103 & BBT & $\mathrm{V}+\mathrm{S}$ & FP, TSAB & 3 & $2-4$ & $24-36$ \\
\hline Hodges 2006 & 6 & BA-S & LD & 6 & $0.4-6000$ & MF & PBST & $\mathrm{SS}$ & 10 & PBST & $\mathrm{V}$ & Plate/FP, TSAB & $2^{(a)}$ & NR & $15-45$ \\
\hline \multirow[t]{2}{*}{ Rose 2004} & 24 & BA-S & LD & 1 & 1937.5 & $\mathrm{Ct}, \mathrm{P}, \mathrm{R}, \mathrm{MF}$ & Dry, PBST & SS & 25.8 & PBST & $\mathrm{U}, \mathrm{V}, \mathrm{S}$ & Plate, TSAB & 1 & NR & 10 \\
\hline & 4 & BA-S & LI & 1 & $1 \mathrm{E}+4 \mathrm{CFU} / \mathrm{swab}$ & $\begin{array}{c}\text { DI: Ct, PE, R, } \\
\text { MF }\end{array}$ & NA & None & NA & PBST & $\mathrm{V}$ & Plate, TSAB & 1 & NR & 10 \\
\hline \multirow[t]{2}{*}{ Frawley 2008} & 6 & BA-S & LD & 1 & $\begin{array}{l}1 \mathrm{E}+2-1 \mathrm{E}+5 \\
\mathrm{CFU} / \text { sample }\end{array}$ & $\mathrm{PE}$ & $\begin{array}{c}\text { Dry, } \\
\text { PBST/Tr }\end{array}$ & $\mathrm{P}, \mathrm{W}, \mathrm{Cl}$ & $1, \mathrm{NR}$ & PBST & $\mathrm{V}$ & Plate, SBA & 1 & NR & $12-24$ \\
\hline & 7 & BA (4) & LD & 1 & $50 \mathrm{CFU} /$ sample & $\mathrm{Ct}$ & $\mathrm{PBST} / \mathrm{Tr}$ & $\begin{array}{c}\mathrm{P}, \mathrm{G}, \mathrm{F}, \mathrm{M}, \mathrm{Cpt}, \\
\text { B, Ct Cloth }\end{array}$ & NR & NR & NR & Plate, NR & 1 & NR & 11 \\
\hline \multirow[t]{3}{*}{ Brown 2007b } & 4 & BAtr & DA & 2 & $10^{2}-10^{3}, 10^{5}-10^{6}$ & $\mathrm{R}$ & DW & SS & 25 & BBT & $\mathrm{S}, \mathrm{H}, \mathrm{V}$ & Plate, PF & 1 & NR & 20 \\
\hline & 1 & BAtr & LI & 1 & 1E+6 CFU/swab & DI: R & NA & None & NA & BBT & $\mathrm{S}, \mathrm{H}, \mathrm{V}$ & Plate, PF & 1 & NR & 20 \\
\hline & 1 & BAtr & LD & 1 & $2.0 \mathrm{E}+5$ & NA & NA & DI: SS & 6.25 & BBT & $\mathrm{S}, \mathrm{H}$ & Plate, BHIA & 1 & NR & 24 \\
\hline \multirow[t]{3}{*}{ Edmonds 2009} & 16 & BAtr & LD & 1 & $1.0 \mathrm{E}+5$ & $\mathrm{Ct}, \mathrm{DP}, \mathrm{R}, \mathrm{MF}$ & DW & $\mathrm{G}$ & 10 & PBSTr & $\mathrm{V}, \mathrm{S}$ & SprP, NR & 1 & $3-4$ & $28-40$ \\
\hline & 16 & BAtr & DA & 1 & $1.0 \mathrm{E}+9$ & $\mathrm{Ct}, \mathrm{DP}, \mathrm{R}, \mathrm{MF}$ & DW & $\mathrm{G}, \mathrm{SS}, \mathrm{PC}, \mathrm{VL}$ & 10 & PBSTr & $\mathrm{V}, \mathrm{S}$ & SprP, NR & 1 & 3 & $24-30$ \\
\hline & 4 & BAtr & LD & 4 & $4.77 \mathrm{E}+3-2.52 \mathrm{E}+6$ & DP & DW & $\mathrm{G}$ & 10 & PBSTr & $\mathrm{V}, \mathrm{S}$ & SprP, NR & 1 & 3 & 30 \\
\hline \multirow[t]{2}{*}{ Valentine 2008} & 16 & $\mathrm{BS}$ & LD & 2 & $9.03 \mathrm{E}+4,2.82 \mathrm{E}+5$ & $\mathrm{Ct}, \mathrm{PF}, \mathrm{PE}, \mathrm{DP}$ & PBST & $\mathrm{P}, \mathrm{O}, \mathrm{PEUF}, \mathrm{Cpt}$ & 104.04 & PBST & $\mathrm{V}$ & Plate, TSA & 1 & 10 & 10 \\
\hline & 4 & $\mathrm{BS}$ & LD & 2 & $9.03 \mathrm{E}+4,2.82 \mathrm{E}+5$ & $\mathrm{Ct}, \mathrm{PF}, \mathrm{PE}, \mathrm{DP}$ & PBST & Monitor & 25 & PBST & $\mathrm{V}$ & Plate, TSA & 1 & 10 & 10 \\
\hline \multirow[t]{3}{*}{ Buttner 2001} & 2 & BS & LD & 1 & $1.48 \mathrm{E}+6$ & SK & PBST & G & 5 & PBT & $\mathrm{V}$ & Plate, TSAC & 1 & 3 & 3 \\
\hline & 8 & BS & DA & 1 & $100-1000$ & $\mathrm{SK}, \mathrm{Ct}$ & PBST & VL, Cpt(3) & 32.49 & PBT & V & Plate, TSAC & 1 & 3 & 3 \\
\hline & 8 & $\mathrm{BS}+\mathrm{PC} 2$ & DA & 1 & $100-1000$ & SK & PBST & VL, Cpt(3) & 32.49 & PBT & $\mathrm{V}$ & Plate, TSAC & 1 & 2 & 2 \\
\hline \multirow[t]{2}{*}{ Buttner 2004a } & 6 & BAtr & DA & 1 & $107.6-1076.4$ & SSPK & NR & VL, W, M & 929 & NR & $\mathrm{HM}$ & Plate, TSA & 1 & 3 & 3 \\
\hline & 3 & BAtr & DA & 1 & $107.6-1076.4$ & SSPK & NR & VL, W, M & 929 & NR & HM & Plate, TSA & 1 & 1 & 2 \\
\hline Buttner 2004b & 4 & BAtr & DA & 1 & NR & $\mathrm{SSPK}, \mathrm{Ct}$ & NR & $\mathrm{M}, \mathrm{W}$ & 100,317 & NR & HM, Shake & Plate, TSA & 1 & NR & 4,8 \\
\hline Quizon 2007 & 4 & BAtr & WA & 1 & NR & PEUF & PBST & PWB, SS, VL, W & 100 & PBST & $\mathrm{V}, \mathrm{S}$ & SP/FP, TSA & 1 & 4 & 4,10 \\
\hline \multirow[t]{4}{*}{ Nellen 2006} & 2 & BS-168 & LD & 1 & $1000 \mathrm{CFU} / \mathrm{swab}$ & DI: Ct, R & NA & NA & NA & PBS & Untreated & Plate, TSA & 1 & 3 & 15 \\
\hline & 6 & BS-168 & LD & 1 & $1000 \mathrm{CFU} / \mathrm{swab}$ & DI: Ct, R & NA & NA & NA & PBS & $\mathrm{V}, \mathrm{S}, \mathrm{V}+\mathrm{S}$ & Plate, TSA & 1 & 3 & 15 \\
\hline & 4 & BS-168 & LD & 1 & 4 & $\mathrm{Ct}, \mathrm{R}$ & DW & PD & 25 & PBS & $\mathrm{NR}, \mathrm{H}+\mathrm{V}+\mathrm{S}$ & Plate, TSA & 1 & 3 & 15 \\
\hline & 10 & BS-168 & $\mathrm{LD}$ & 1 & 16 & $\mathrm{R}$ & DW & (b) & 25 & PBS & $\mathrm{NR}, \mathrm{H}+\mathrm{V}+\mathrm{S}$ & Plate, TSA & 1 & 3 & 3 \\
\hline \multirow[t]{2}{*}{ Hodges 2010} & 6 & BA-S & LD & 3 & $1.4,15.3,1607.2$ & MF & $\begin{array}{l}\text { PBST, } \\
\text { PBST+ }\end{array}$ & SS & 26 & PBST & V & FP, TSAB & 12 & 1 & $118-120$ \\
\hline & 6 & BA-S & LI & 3 & 36-33300 CFU/swab & DI: MF & NA, PBST+ & NA & NA & PBST & $\mathrm{V}$ & FP, TSAB & 12 & 1 & $24-48$ \\
\hline
\end{tabular}

(a) Five analysts total from two laboratories.

(b) Aluminum, V2A steel, MLI foil, Kapton ${ }^{\circledR}$, Teflon $^{\circledR}$ 
Table 2b. Summary of test results for swab sampling. Acronyms and abbreviations are defined in Table 6.

\begin{tabular}{|c|c|c|c|c|c|c|c|c|c|c|c|c|c|c|c|c|}
\hline \multirow[b]{2}{*}{$\begin{array}{l}\text { Reference } \\
\text { (lead author } \\
\text { and year) }\end{array}$} & \multirow[b]{2}{*}{$\begin{array}{c}\# \\
\text { tests }\end{array}$} & \multicolumn{5}{|c|}{$\begin{array}{c}\text { Recovery concentration results - } \\
\text { Mean \& } \% \text { RSDs } \\
\end{array}$} & \multicolumn{5}{|c|}{$\begin{array}{c}\text { Recovery efficiency (RE) - } \\
\text { Mean \& \%RSDs } \\
\end{array}$} & \multicolumn{5}{|c|}{ LOD, FNR, and FPR } \\
\hline & & $\begin{array}{c}\text { Mean } \\
\left(\mathrm{CFU} / \mathrm{cm}^{2}\right)\end{array}$ & $\begin{array}{l}\text { Lab } \\
\% \text { RSD }\end{array}$ & $\begin{array}{c}\text { Run } \\
\% \text { RSD }\end{array}$ & $\begin{array}{l}\text { Sample- } \\
\text { within- } \\
\text { run } \\
\% \text { RSD }\end{array}$ & $\begin{array}{c}\text { Total } \\
\% \text { RSD }\end{array}$ & $\begin{array}{c}\mathrm{RE} \\
\text { mean } \\
(\%)\end{array}$ & $\begin{array}{l}\text { RE lab } \\
\text { \%RSD }\end{array}$ & $\begin{array}{l}\text { RE run } \\
\text { \%RSD }\end{array}$ & $\begin{array}{c}\text { RE } \\
\text { sample- } \\
\text { within- } \\
\text { run } \\
\% \text { RSD }\end{array}$ & $\begin{array}{c}\mathrm{RE} \\
\text { total } \\
\% \mathrm{RSD} \\
\end{array}$ & $\begin{array}{c}\text { Positive } \\
\text { result } \\
(\mathrm{CFU})\end{array}$ & $\begin{array}{c}\text { LOD } \\
\text { defi- } \\
\text { nition }\end{array}$ & $\begin{array}{c}\text { LOD } \\
\left(\mathrm{CFU} / \mathrm{cm}^{2}\right)\end{array}$ & FNR & FPR \\
\hline Estill 2009 & 6 & $0.0012-0.18$ & $0-110$ & $0-98$ & $57-460$ & $73-473$ & $3.4-14.0$ & $0-110$ & $0-110$ & $76-550$ & $81-560$ & $\geq 1$ & $\mathrm{LOD}_{95}$ & $\begin{array}{l}0.4(\mathrm{SS}) \\
1.9(\mathrm{Cpt}) \\
\end{array}$ & NR & 0.037 \\
\hline Hodges 2006 & 6 & $0.1-2900$ & NR & NR & $27-100$ & NR & $31.7-49.1$ & NA & NA, $28-33$ & $21-91$ & NA & $\geq 1$ & $\mathrm{LOD}_{90}$ & 1.2 & $0-0.27$ & NR \\
\hline \multirow[t]{2}{*}{ Rose 2004} & 24 & NR & NA & NR & NR & NA & $0.1-43.6$ & NA & NR & $18-200$ & NA & NR & NR & NR & NR & NR \\
\hline & 4 & NR & NA & NR & NR & NA & $83.8-93.9$ & NA & NR & $7-12$ & NA & NR & NR & NR & NR & NR \\
\hline \multirow[t]{2}{*}{ Frawley 2008} & 6 & NR & NA & NR & NR & NA & $0.2-5.5$ & NA & NR & $30-63$ & NA & NR & NR & NR & NR & NR \\
\hline & 7 & NR & NA & NR & NR & NA & $0-15$ & NA & NR & $28-144$ & NA & NR & NR & NR & NR & NR \\
\hline \multirow[t]{3}{*}{ Brown 2007b } & 4 & NR & NA & NR & NR & NA & $35.5-45.6$ & NA & NR & $34-67$ & NA & $\geq 1$ & NR & 1 & NR & NR \\
\hline & 1 & NR & NA & NR & NR & NA & 75.6 & NA & NR & 16 & NA & NR & NR & NR & NR & NR \\
\hline & 1 & NR & NA & NR & NR & NA & 99.9 & NA & NR & 0.1 & NA & NR & NR & NR & NR & NR \\
\hline \multirow[t]{3}{*}{ Edmonds 2009} & 16 & NR & NA & NR & NR & NA & $42.5-89.1$ & NA & $11-33$ & $8-16$ & NA & NR & NR & NR & NR & NR \\
\hline & 16 & NR & NA & NR & NR & NA & $51.5-75.5$ & NA & $10-35$ & $8-20$ & NA & NR & NR & NR & NR & NR \\
\hline & 4 & $2.0 \mathrm{E}+2-2.4 \mathrm{E}+6$ & NA & $19-29^{(a)}$ & (a) & NA & $42.1-92.7$ & NA & NR & $19-29$ & NA & NR & NR & NR & NR & NR \\
\hline \multirow[t]{2}{*}{ Valentine 2008} & 16 & NR & NA & NR & NR & NA & $0.6-6.6$ & NA & NR & $25-150$ & NA & NR & NR & NR & NR & NR \\
\hline & 4 & NR & NA & NR & NR & NA & $0.5-2.7$ & NA & NR & $18-84$ & NA & NR & NR & NR & NR & NR \\
\hline \multirow[t]{3}{*}{ Buttner 2001} & 2 & NR & NA & NR & NR & NA & $68.6-73.5$ & NA & NR & $6-7$ & NA & NR & NR & NR & NR & NR \\
\hline & 8 & $82-748$ & NA & $7-107^{(\mathrm{a})}$ & (a) & NA & NR & NA & NR & NR & NA & NR & NR & NR & NR & NR \\
\hline & 8 & $84-1264$ & NA & $18-85^{\text {(a) }}$ & (a) & NA & NR & NA & NR & NR & NA & NR & NR & NR & NR & NR \\
\hline \multirow[t]{2}{*}{ Buttner 2004a } & 6 & $340-711$ & NA & $24-92^{(a)}$ & (a) & NA & NR & NA & NR & NR & NA & NR & NR & NR & NR & NR \\
\hline & 3 & $523-704$ & NA & NR & $8-55$ & NA & NR & NA & NR & NR & NA & NR & NR & NR & NR & NR \\
\hline Buttner 2004b & 4 & $840-1200$ & NA & NR & $11-28$ & NA & NR & NA & NR & NR & NA & NR & NR & NR & NR & NR \\
\hline & 4 & $4170-5670$ & NA & $17-36^{(\mathrm{a})}$ & (a) & NA & NR & NA & NR & NR & NA & NR & NR & NR & NR & NR \\
\hline \multirow[t]{4}{*}{ Nellen 2006} & 2 & 0.29 & NA & $17^{(\mathrm{a})}$ & (a) & NA & 13.4 & NA & $17-18^{(\mathrm{a})}$ & (a) & NA & NR & NR & NR & NR & NR \\
\hline & 6 & $1.42-2.04$ & NA & $1-6^{(a)}$ & (a) & NA & $65.8-94.5$ & NA & $3-7^{(a)}$ & (a) & NA & NR & NR & NR & NR & NR \\
\hline & 4 & $0.95-1.75$ & NA & $25-33^{(a)}$ & (a) & NA & $35.9-66.1$ & NA & $27-34^{(\mathrm{a})}$ & (a) & NA & NR & NR & NR & NR & NR \\
\hline & 10 & $0.73-2.72$ & NA & $24-56^{(\mathrm{a})}$ & (a) & NA & $19.8-73.7$ & NA & $26-52^{(a)}$ & (a) & NA & NR & NR & NR & NR & NR \\
\hline \multirow[t]{2}{*}{ Hodges 2010} & 6 & $0.48-1064.32$ & NR & NR & NR & NR & $15.8-83.1$ & $12.1-24.2$ & (b) & $9-57^{(\mathrm{b})}$ & $15-59$ & (c) & NR & 0.8 & 0 & $0-0.083$ \\
\hline & 6 & $0.71-87.27$ & NR & NR & NR & NR & $27.9-73.9$ & $26.7-46.8$ & (b) & $15-40^{(\mathrm{b})}$ & $32-57$ & (c) & NR & NR & $0-0.17$ & $0-0.146$ \\
\hline
\end{tabular}

(a) Combined estimate of "run" and "sample-within-run" uncertainties.

(b) The precision (variance within laboratories) reported by Hodges et al. (2010) is listed in this table as sample-within-run uncertainty.

(c) $\geq 1 \mathrm{CFU} / \mathrm{ml}$ 
Table 3a. Summary of test conditions for wipe sampling studies. Acronyms and abbreviations are defined in Table 6 and the footnotes.

\begin{tabular}{|c|c|c|c|c|c|c|c|c|c|c|c|c|c|c|c|}
\hline \multirow{2}{*}{$\begin{array}{l}\text { Reference } \\
\text { (lead author } \\
\text { and year) }\end{array}$} & \multirow[b]{2}{*}{$\begin{array}{c}\# \\
\text { tests }\end{array}$} & \multirow[b]{2}{*}{ Agent } & \multirow{2}{*}{$\begin{array}{l}\text { Agent } \\
\text { depo- } \\
\text { sition }\end{array}$} & \multicolumn{2}{|r|}{$\begin{array}{c}\text { Agent } \\
\text { concentration(s) }\end{array}$} & \multirow[b]{2}{*}{ Wipe types } & \multirow[b]{2}{*}{$\begin{array}{l}\text { Wetting } \\
\text { agent }\end{array}$} & \multirow[b]{2}{*}{ Surface types } & \multirow{2}{*}{$\begin{array}{l}\text { Surface } \\
\text { area } \\
\text { sampled } \\
\left(\mathrm{cm}^{2}\right)\end{array}$} & \multirow{2}{*}{$\begin{array}{l}\text { Extract } \\
\text {-ion } \\
\text { liquid }\end{array}$} & \multirow[b]{2}{*}{$\begin{array}{l}\text { Extraction } \\
\text { method }\end{array}$} & \multirow{2}{*}{$\begin{array}{l}\text { Culture } \\
\text { method, } \\
\text { medium }\end{array}$} & \multirow[b]{2}{*}{$\begin{array}{c}\# \\
\text { labs }\end{array}$} & \multirow[b]{2}{*}{$\begin{array}{l}\# \text { test } \\
\text { runs }\end{array}$} & \multirow{2}{*}{$\begin{array}{c}\# \\
\text { samples } \\
\text { /test }\end{array}$} \\
\hline & & & & \# & $\begin{array}{c}\text { Range } \\
\left(\mathrm{CFU} / \mathrm{cm}^{2}\right)\end{array}$ & & & & & & & & & & \\
\hline \multirow[t]{2}{*}{ Estill 2009} & 3 & BA-S & $\mathrm{DA}$ & 3 & $0.03-2$ & Sponge & BBT & SS & 929 & BBT & $\mathrm{A}+\mathrm{C}+\mathrm{V}+\mathrm{S}$ & FP, TSAB & 3 & 3 & 27 \\
\hline & 3 & BA-S & DA & 3 & $0.03-2$ & Sponge & BBT & $\mathrm{Cpt}$ & 929 & BBT & $\mathrm{A}+\mathrm{C}+\mathrm{V}+\mathrm{S}$ & FP, TSAB & 3 & $2-4$ & $18-36$ \\
\hline \multirow[t]{3}{*}{ Brown 2007a } & 4 & $\mathrm{BA}$ & DA & 2 & $10^{2}-10^{3}, 10^{4}-10^{5}$ & PR gauze & DW & SS, PWB & 25 & BBT & $\mathrm{S}+\mathrm{H}+\mathrm{V}$ & Plate, PF & 1 & NR & 20 \\
\hline & 1 & $\mathrm{BA}$ & LI & 1 & NR & DI: PR gauze & DW & None & NA & BBT & $\mathrm{S}+\mathrm{H}+\mathrm{V}$ & Plate, PF & 1 & NR & 40 \\
\hline & 1 & $\mathrm{BA}$ & LD & 1 & $2.0 \mathrm{E}+5$ & NA & NA & DI: SS & 25 & BBT & $\mathrm{S}+\mathrm{H}$ & Plate, BHIA & 1 & NR & 24 \\
\hline \multirow[t]{2}{*}{ Buttner 2001} & 1 & $\begin{array}{c}\mathrm{BS} \\
\mathrm{PS}^{(\mathrm{a})}\end{array}$ & LD & 1 & $1.48 \mathrm{E}+7$ & Sponge & PBST & Glass & 32.49 & PBST & $\mathrm{HS}$ & Plate, TSAC & 1 & 3 & 3 \\
\hline & 12 & $\begin{array}{l}\mathrm{BS}+\mathrm{PC} 2 \\
\mathrm{BS}+\mathrm{PC} 4\end{array}$ & DA & 1 & $1 E+2-1 E+3$ & Sponge & PBST & $\begin{array}{l}\text { VL, Cpt-R, } \\
\text { Cpt-S, Cpt-C }\end{array}$ & 32.49 & PBST & HS & Plate, TSAC & 1 & 2,3 & 2,3 \\
\hline \multirow[t]{2}{*}{ Valentine 2008} & 12 & BS & LD & 1 & 90349.9 & $\mathrm{Ct}, \mathrm{HCW}, \mathrm{PR}$ & PBST & $\mathrm{P}, \mathrm{O}, \mathrm{PEUF}, \mathrm{Cpt}$ & 104.04 & PBST & $\mathrm{V}+\mathrm{C}$ & Plate, TSAC & 1 & 10 & 10 \\
\hline & 3 & $\mathrm{BS}$ & LD & 1 & 282,000 & $\mathrm{Ct}, \mathrm{HCW}, \mathrm{PR}$ & PBST & Monitor & 25 & PBST & $\mathrm{V}+\mathrm{C}$ & Plate, TSAC & 1 & 10 & 10 \\
\hline \multirow[t]{2}{*}{ Buttner 2004a } & 12 & BAtr & $\overline{D A}$ & 1 & $107.6-1076.4$ & Swipe, HW & PBST & $\mathrm{VL}, \mathrm{W}, \mathrm{M}$ & 929 & NR & HM & Plate, TSA & 1 & 3 & 3 \\
\hline & 6 & BAtr & DA & 1 & $107.6-1076.4$ & Swipe, HW & PBST & VL W, M & 929 & NR & $\mathrm{HM}$ & Plate, TSA & 1 & 1 & 1,2 \\
\hline \multirow[t]{2}{*}{ Buttner 2004b } & 4 & BAtr & LI & 2 & $10,18.2$ & BiSKit & Dry, PBST & $\mathrm{M}$ & 10,000 & PBST & BiSKit & Plate, TSA & 1 & NR & 4,8 \\
\hline & 6 & BAtr & DA & 1 & NR & BiSKit & $\begin{array}{c}\text { Dry, PBST, } \\
\text { NR }\end{array}$ & $\mathrm{M}, \mathrm{W}$ & 10,000 & PBST & BiSKit & Plate, TSA & 1 & NR & 4,8 \\
\hline Frawley 2008 & 6 & BA-S & LD & 1 & $1 \mathrm{E}+2-1 \mathrm{E}+5 /$ sample & Gauze & $\begin{array}{c}\text { Dry, } \\
\text { PBST/Tr }\end{array}$ & $\mathrm{P}, \mathrm{W}, \mathrm{Ct}$ Cloth & $1, \mathrm{NR}$ & PBST & $\mathrm{V}$ & Plate, SBA & 1 & NR & 12,24 \\
\hline Einfeld 2011 & 10 & $\begin{array}{l}\text { BAtr } \\
\text { BAtr+ } \\
\text { Grime }\end{array}$ & DA & 1 & $9,55 \mathrm{E}+0-6.81 \mathrm{E}+4$ & PR & $\begin{array}{c}\mathrm{DW} \\
\mathrm{RH}=10-15, \\
82-90\end{array}$ & SS, G, Marble & 100 & BBT & $\mathrm{S}+\mathrm{H}+\mathrm{V}$ & Plate, PF & 1 & $2,3,5$ & $24-60$ \\
\hline Quizon 2007 & 4 & BAtr & WA & 1 & NR & PR & PBST & PWB, SS, VL, W & 900 & PBST & $\mathrm{C}+\mathrm{V}+\mathrm{S}$ & SP/FP, TSA & 1 & 4 & 4,10 \\
\hline \multirow[t]{2}{*}{ Krauter 2011} & 27 & BAtr & LD & 8 & $0.00248-1.85380$ & Sponge stick & NB & SS, CerT, VL & 645.16 & PBST & $\mathrm{St}+\mathrm{C}+\mathrm{V}+\mathrm{S}$ & (b) & 1 & 1 & 9,10 \\
\hline & 27 & BAtr & LD & 8 & $0.00775-0.15371$ & Sponge stick & NB & FL, PW, PLCP & 645.16 & PBST & $\mathrm{St}+\mathrm{C}+\mathrm{V}+\mathrm{S}$ & (b) & 1 & 1 & 9,10 \\
\hline \multirow[t]{7}{*}{ Rose 2011} & 3 & BA-S & LS & 3 & $0.01349-17.123$ & DI: Sp. stick & $\mathrm{NB}+\mathrm{ATD}$ & SS & 645.16 & PBST & $\mathrm{St}+\mathrm{C}$ & SP/FP, TSAB & 9 & 1 & $17-18$ \\
\hline & 3 & BA-S & LS & 3 & $0.04046-51.367$ & Sponge stick & NB+ATD & SS & 645.16 & PBST & $\mathrm{St}+\mathrm{C}$ & SP/FP, TSAB & 9 & 1 & $54-63$ \\
\hline & 1 & BA-S & LS & 1 & 15.5 & Rayon gauze & PBST & SS & 645.16 & PBST & $\mathrm{St}+\mathrm{C}$ & SP/FP, TSAB & 1 & 1 & 10 \\
\hline & 1 & BA-S & LS & 1 & 15.5 & Sponge wipe & DE broth & SS & 645.16 & PBST & $\mathrm{St}+\mathrm{C}$ & SP/FP, TSAB & 1 & 1 & 10 \\
\hline & 1 & BA-S & LS & 1 & 15.5 & Sponge wipe & BB & SS & 645.16 & PBST & $\mathrm{St}+\mathrm{C}$ & SP/FP, TSAB & 1 & 1 & 10 \\
\hline & 1 & BA-S & LS & 1 & 15.5 & Sponge stick & PBST & SS & 645.16 & PBST & $\mathrm{St}+\mathrm{C}$ & $\mathrm{SP} / \mathrm{FP}, \mathrm{TSAB}$ & 1 & 1 & 10 \\
\hline & 3 & BA-S & LS & 3 & $0.0155,0.155,15.5$ & PEF sponge & PBST & SS & 645.16 & PBST & $\mathrm{St}+\mathrm{C}$ & SP/FP, TSAB & 1 & 3 & 15 \\
\hline $\begin{array}{l}\text { Montgomery } \\
2008\end{array}$ & 4 & BA-S & EWD & 1 & NR & $\begin{array}{l}\text { PR gauze, pad } \\
2 \text { wipe tech's }\end{array}$ & PBSTr & HVAC filter & 100 & PBSTr & Shake $+\mathrm{C}$ & Plate, NR & 1 & 2,3 & $2-5$ \\
\hline
\end{tabular}

(a) PC2 and PC4 = Penicillium chrysogenum at $10^{2}$ and $10^{4} \mathrm{CFU} / \mathrm{cm}^{2}$ on test coupons.

(b) Plate with growth medium, or filter plate with TSA. 
Table 3b. Summary of test results for wipe sampling. Acronyms and abbreviations are defined in Table 6 .

\begin{tabular}{|c|c|c|c|c|c|c|c|c|c|c|c|c|c|c|c|c|}
\hline \multirow[b]{2}{*}{$\begin{array}{l}\text { Reference } \\
\text { (lead author } \\
\text { and year) }\end{array}$} & \multirow[b]{2}{*}{$\begin{array}{c}\# \\
\text { tests }\end{array}$} & \multicolumn{5}{|c|}{$\begin{array}{c}\text { Recovery concentration results - } \\
\text { Mean \& \%RSDs }\end{array}$} & \multicolumn{5}{|c|}{$\begin{array}{c}\text { Recovery efficiency (RE) - } \\
\text { Mean \& \%RSDs }\end{array}$} & \multicolumn{5}{|c|}{ LOD, FNR, and FPR } \\
\hline & & $\begin{array}{c}\text { Mean } \\
\left(\mathrm{CFU} / \mathrm{cm}^{2}\right)\end{array}$ & $\begin{array}{l}\text { Lab } \\
\% \text { RSD }\end{array}$ & $\begin{array}{c}\text { Run } \\
\% \text { RSD }\end{array}$ & $\begin{array}{l}\text { Sample- } \\
\text { within- } \\
\text { run } \\
\% \text { RSD }\end{array}$ & $\begin{array}{c}\text { Total } \\
\% \text { RSD }\end{array}$ & $\begin{array}{c}\mathrm{RE} \\
\text { mean } \\
(\%)\end{array}$ & $\begin{array}{l}\text { RE lab } \\
\% \text { RSD }\end{array}$ & $\begin{array}{l}\text { RE run } \\
\% \text { RSD }\end{array}$ & $\begin{array}{c}\mathrm{RE} \\
\text { sample- } \\
\text { within-run } \\
\% \text { RSD }\end{array}$ & $\begin{array}{c}\mathrm{RE} \\
\text { total } \\
\% \mathrm{RSD}\end{array}$ & $\begin{array}{c}\text { Positive } \\
\text { result } \\
\text { (CFU) }\end{array}$ & $\begin{array}{c}\text { LOD } \\
\text { defi- } \\
\text { nition }\end{array}$ & $\begin{array}{c}\text { LOD } \\
\left(\mathrm{CFU} / \mathrm{cm}^{2}\right)\end{array}$ & FNR & FPR \\
\hline \multirow[t]{2}{*}{ Estill 2009} & 3 & $0.017-0.350$ & $9-27$ & $0-45$ & $61-200$ & $80-200$ & $18-31$ & $14-29$ & $0-13$ & $53-160$ & $57-160$ & $\geq 3$ & $\mathrm{LOD}_{95}$ & 0.15 & NR & 0.133 \\
\hline & 3 & $0.084-0.500$ & $9-26$ & $0-52$ & $37-180$ & $60-182$ & $21-120$ & $0-11$ & $0-63$ & $40-220$ & $75-220$ & $\geq 1$ & $\mathrm{LOD}_{95}$ & 0.009 & NR & 0.0 \\
\hline \multirow[t]{3}{*}{ Brown 2007a } & 4 & NR & NA & NR & NR & NA & $25.2-39.2$ & NA & NR & $32-59$ & NA & $\geq 1 \mathrm{CFU} / \mathrm{ml}$ & NR & $3.6-4.2$ & NR & NR \\
\hline & 1 & NR & NA & NR & NR & NA & 93.2 & NA & NR & 9 & NA & $\geq 1 \mathrm{CFU} / \mathrm{ml}$ & NR & NR & NR & NR \\
\hline & 1 & NR & NA & NR & NR & NA & 99.9 & NA & NR & 0.1 & NA & $\geq 1 \mathrm{CFU} / \mathrm{ml}$ & NR & NR & NR & NR \\
\hline \multirow[t]{2}{*}{ Buttner 2001} & 1 & NR & NA & NR & NR & NA & 74.3 & NA & NR & 7 & NA & NR & NR & NR & NR & NR \\
\hline & 12 & $127-1283$ & NA & $0.6-101^{\text {(a) }}$ & (a) & NA & NR & NA & NR & NR & NA & NR & NR & NR & NR & NR \\
\hline \multirow[t]{2}{*}{ Valentine 2008} & 12 & NR & NA & NR & NR & NA & $1.4-7.9$ & NA & NR & $18-84$ & NA & NR & NR & NR & NR & NR \\
\hline & 3 & NR & NA & NR & NR & NA & $2.6-4.2$ & NA & NR & $22-38$ & NA & NR & NR & NR & NR & NR \\
\hline \multirow[t]{2}{*}{ Buttner 2004a } & 12 & $711-6950$ & NA & $0-44^{(\mathrm{a})}$ & (a) & NA & NR & NA & NR & NR & NA & NR & NR & NR & NR & NR \\
\hline & 6 & $506-883$ & NA & NA & $3-95$ & NA & NR & NA & NR & NR & NA & NR & NR & NR & NR & NR \\
\hline \multirow[t]{2}{*}{ Buttner 2004b } & 4 & $2.05-3.35$ & NA & NR & NR & NA & $11.3-18.4$ & NA & NR & NR & NA & $\geq 1 \mathrm{CFU} / \mathrm{ml}$ & NR & $0.0042-0.01$ & NR & NR \\
\hline & 6 & $115-590$ & NA & $14-84$ & NR & NA & NR & NA & NR & NR & NA & NR & NR & NR & NR & NR \\
\hline Frawley 2008 & 6 & NR & NA & NR & NR & NA & $0.2-6.6$ & NA & NR & $46-70$ & NA & NR & NR & NR & NR & NR \\
\hline Einfeld 2011 & 10 & NR & NA & NR & NR & NA & $18.0-96.8$ & NA & $29-96^{(a)}$ & (a) & NA & NR & NR & NR & NR & NR \\
\hline Quizon 2007 & 4 & $6670-14615$ & NA & $8-20^{(a)}$ & (a) & NA & $39.5, \mathrm{NR}$ & NA & NR & NR & NA & NR & NR & NR & NR & NR \\
\hline \multirow[t]{2}{*}{ Krauter 2011} & 27 & $0.0003-0.994$ & NA & NR & $\begin{array}{c}6.6- \\
316\end{array}$ & NA & $12.5-75.5$ & NA & NR & $6.6-316$ & NA & $\geq 1$ & $\begin{array}{l}\operatorname{LOD}_{95} \\
\operatorname{LOD}_{90}\end{array}$ & $0.013-0.038$ & $0-0.933$ & 0.0 \\
\hline & 27 & $0.0-0.084$ & NA & NR & $0-316$ & NA & $4.0-76.9$ & NA & NR & $0-316$ & NA & $\geq 1$ & $\begin{array}{l}\operatorname{LOD}_{95} \\
\operatorname{LOD}_{90}\end{array}$ & $0.015-0.051$ & $0-1.0$ & 0.0 \\
\hline \multirow[t]{7}{*}{ Rose 2011} & 3 & $0.0064-13.34$ & NR & NR & NR & NR & $46.1-77.9$ & $14-48$ & NA & $11-54$ & $18-72$ & $\geq 1$ & NR & NR & $0-0.159$ & $0-0.056$ \\
\hline & 3 & $0.013-15.48$ & NR & NR & NR & NR & $24.4-32.4$ & $20-31$ & NA & $20-69$ & $28-76$ & $\geq 1$ & NR & 0.031 & NR & NR \\
\hline & 1 & NR & NR & NR & NR & NR & 30.8 & NA & NA & 50 & NA & NR & NR & NR & NR & NR \\
\hline & 1 & NR & NR & NR & NR & NR & 32.3 & NA & NA & 30 & NA & NR & NR & NR & NR & NR \\
\hline & 1 & NR & NR & NR & NR & NR & 26.8 & NA & NA & 21 & NA & NR & NR & NR & NR & NR \\
\hline & 1 & NR & NR & NR & NR & NR & 36.3 & NA & NA & 25 & NA & NR & NR & NR & NR & NR \\
\hline & 3 & NR & NR & NR & NR & NR & 26.0 & NA & NA & 38 & NA & NR & NR & NR & NR & NR \\
\hline $\begin{array}{l}\text { Montgomery } \\
2008\end{array}$ & 4 & NR & NA & NR & NR & NA & $14.5-19.9$ & NA & $10-37$ & $0-27$ & NA & NR & NR & NR & NR & NR \\
\hline
\end{tabular}

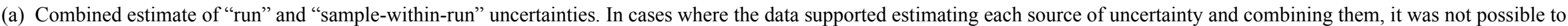
tell whether these calculations were correctly implemented. A common, but incorrect, method is to calculate a single standard deviation from data including two or more sources of uncertainty. 
Table 4a. Summary of test conditions for vacuum sampling studies. Acronyms and abbreviations are defined in Table 6 and the footnotes.

\begin{tabular}{|c|c|c|c|c|c|c|c|c|c|c|c|c|c|c|c|c|}
\hline \multirow{2}{*}{$\begin{array}{l}\text { Reference } \\
\text { (lead author } \\
\text { and year) }\end{array}$} & \multirow[b]{2}{*}{$\begin{array}{c}\# \\
\text { tests }\end{array}$} & \multirow[b]{2}{*}{ Agent } & \multirow{2}{*}{$\begin{array}{l}\text { Agent } \\
\text { depo- } \\
\text { sition }\end{array}$} & \multicolumn{2}{|r|}{$\begin{array}{c}\text { Agent } \\
\text { concentration(s) }\end{array}$} & \multirow[b]{2}{*}{$\begin{array}{l}\text { Vacuum } \\
\text { filter type }\end{array}$} & \multirow{2}{*}{$\begin{array}{l}\text { Vacuum } \\
\text { technique } \\
\text { (a) }\end{array}$} & \multirow{2}{*}{$\begin{array}{c}\text { Relative } \\
\text { humidity } \\
(\%)\end{array}$} & \multirow[b]{2}{*}{$\begin{array}{c}\text { Surface } \\
\text { Types }\end{array}$} & \multirow{2}{*}{$\begin{array}{l}\text { Surface } \\
\text { area } \\
\text { sampled } \\
\left(\mathrm{cm}^{2}\right)\end{array}$} & \multirow{2}{*}{$\begin{array}{l}\text { Extract } \\
\text {-ion } \\
\text { liquid }\end{array}$} & \multirow{2}{*}{$\begin{array}{c}\text { Extraction } \\
\text { method }\end{array}$} & \multirow{2}{*}{$\begin{array}{l}\text { Culture } \\
\text { method, } \\
\text { medium }\end{array}$} & \multirow[b]{2}{*}{$\begin{array}{c}\# \\
\text { labs }\end{array}$} & \multirow[b]{2}{*}{$\begin{array}{l}\text { \# test } \\
\text { runs }\end{array}$} & \multirow{2}{*}{$\begin{array}{c}\# \\
\text { samples } \\
\text { /test }\end{array}$} \\
\hline & & & & \# & $\begin{array}{c}\text { Range } \\
\left(\mathrm{CFU} / \mathrm{cm}^{2}\right)\end{array}$ & & & & & & & & & & & \\
\hline \multirow[t]{2}{*}{ Estill 2009} & 3 & BA-S & DA & 3 & $0.03-2$ & HEPA sock & P2D & $\mathrm{NR}$ & SS & 929 & BBT & $\mathrm{A}+\mathrm{C}+\mathrm{V}+\mathrm{S}$ & FP, TSAB & 1 & 3 & 27 \\
\hline & 3 & BA-S & DA & 3 & $0.03-2$ & HEPA sock & $\mathrm{P} 2 \mathrm{D}$ & NR & $\mathrm{Cpt}$ & 929 & BBT & $\mathrm{A}+\mathrm{C}+\mathrm{V}+\mathrm{S}$ & FP, TSAB & 1 & $2-4$ & $18-36$ \\
\hline Brown 2007c & 8 & BA & DA & 2 & $\begin{array}{l}1 \mathrm{E}+2-1 \mathrm{E}+3 \\
1 \mathrm{E}+4-1 \mathrm{E}+5\end{array}$ & HEPA filter & PP2D & NR & $\begin{array}{c}\text { SS, PWB, } \\
\text { Cpt, Concrete }\end{array}$ & 100 & BBT & $\mathrm{S}+\mathrm{H}+\mathrm{V}$ & Plate, PF & 1 & NR & $13-24$ \\
\hline Einfeld 2011 & 6 & $\begin{array}{c}\text { BAtr, } \\
\text { BAtr } \\
+ \text { grime } \\
\end{array}$ & DA & 1 & $\begin{array}{c}1.31 \mathrm{E}+3- \\
8.78 \mathrm{E}+4\end{array}$ & PE filter & NR & $\begin{array}{c}36-48 \\
77-90\end{array}$ & $\begin{array}{l}\text { Marble, } \\
\text { Concrete }\end{array}$ & 100 & BBT & $\mathrm{S}+\mathrm{H}+\mathrm{V}$ & Plate, PF & 1 & $3-5$ & $33-59$ \\
\hline Quizon 2007 & 6 & BAtr & WA & 1 & NR & HEPA sock & SVH & NR & $\begin{array}{l}\text { CelT, PWB, } \\
\text { SS, VL, W }\end{array}$ & 900 & PBST & $\mathrm{C}+\mathrm{V}+\mathrm{S}$ & SP/FP, TSA & 1 & 4 & $4-10$ \\
\hline \multirow[t]{4}{*}{$\begin{array}{l}\text { Montgomery } \\
2008\end{array}$} & 1 & BA-S & EWD & 1 & $7.98 \mathrm{E}+2$ & HEPA sock & $\mathrm{R}$ & NR & HVAC filter & 309.68 & PBSTr & Shake $+\mathrm{C}$ & NR, NR & 1 & 3 & NR \\
\hline & 4 & BA-S & EWD & 3 & $\begin{array}{c}7.98 \mathrm{E}+2, \\
9.0 \mathrm{E}+4,9.1 \mathrm{E}+6\end{array}$ & TECF & $\mathrm{G}, \mathrm{R}$ & NR & HVAC filter & 400 & PBSTr & Shake $+\mathrm{C}$ & $\mathrm{NR}, \mathrm{NR}$ & 1 & 3 & $9, \mathrm{NR}$ \\
\hline & 3 & BA-S & EWD & 2 & 167,201 & TECF & VS, TD & $\mathrm{Nl}$ & $\mathrm{Cp}$ & 400 & PBSTr & Shak & NR, NR & 1 & 1 & 3,6 \\
\hline & 2 & BA-S & WD & 1 & 111 & TECF & VS, TP & NR & $\mathrm{Cpt}$ & 400 & PBSTr & Shake $+\mathrm{C}$ & NR, NR & 1 & 1 & 3 \\
\hline
\end{tabular}

二 $\quad$ (a) $\mathrm{R}=$ rough, $\mathrm{G}=$ gentle, $\mathrm{VS}=$ vertical slide, $\mathrm{TD}=$ tilt drag, $\mathrm{TP}=$ tilt push. See Table 6 for remaining acronyms.

Table 4b. Summary of test results for vacuum sampling. Acronyms and abbreviations are defined in Table 6.

\begin{tabular}{|c|c|c|c|c|c|c|c|c|c|c|c|c|c|c|c|c|}
\hline \multirow[b]{2}{*}{$\begin{array}{l}\text { Reference } \\
\text { (lead author and } \\
\text { year) }\end{array}$} & \multirow[b]{2}{*}{$\begin{array}{c}\# \\
\text { tests }\end{array}$} & \multicolumn{5}{|c|}{$\begin{array}{c}\text { Recovery concentration results - } \\
\text { Mean \& \%RSDs }\end{array}$} & \multicolumn{5}{|c|}{$\begin{array}{c}\text { Recovery efficiency }(\mathrm{RE})- \\
\text { Mean \& \%RSDs }\end{array}$} & \multicolumn{5}{|c|}{ LOD, FNR, and FPR } \\
\hline & & $\begin{array}{c}\text { Mean } \\
\left(\mathrm{CFU} / \mathrm{cm}^{2}\right)\end{array}$ & $\begin{array}{l}\text { Lab } \\
\% \text { RSD }\end{array}$ & $\begin{array}{c}\text { Run } \\
\% \text { RSD }\end{array}$ & $\begin{array}{l}\text { Sample- } \\
\text { within- } \\
\text { run } \\
\% \mathrm{RSD}\end{array}$ & $\begin{array}{c}\text { Total } \\
\% \text { RSD } \\
\end{array}$ & $\begin{array}{c}\mathrm{RE} \\
\text { mean } \\
(\%)\end{array}$ & $\begin{array}{l}\text { RE lab } \\
\% \text { RSD }\end{array}$ & $\begin{array}{l}\text { RE run } \\
\% \text { RSD }\end{array}$ & $\begin{array}{l}\text { RE sample- } \\
\text { within-run } \\
\% \text { RSD } \\
\end{array}$ & $\begin{array}{c}\mathrm{RE} \\
\text { total } \\
\% \mathrm{RSD}\end{array}$ & $\begin{array}{l}\text { Positive } \\
\text { result } \\
(\mathrm{CFU})\end{array}$ & $\begin{array}{c}\text { LOD } \\
\text { defi- } \\
\text { nition }\end{array}$ & $\begin{array}{c}\text { LOD } \\
\left(\mathrm{CFU} / \mathrm{cm}^{2}\right)\end{array}$ & FNR & FPR \\
\hline Estill 2009 & $\begin{array}{l}3 \\
3 \\
\end{array}$ & $\begin{array}{c}0.0043-0.1 \\
0.0011-0.062 \\
\end{array}$ & $\begin{array}{c}18-38 \\
0-30 \\
\end{array}$ & $\begin{array}{l}0-50 \\
0-57\end{array}$ & $\begin{array}{c}82-98 \\
44-170 \\
\end{array}$ & $\begin{array}{l}84-116 \\
65-170 \\
\end{array}$ & $\begin{array}{l}3.7-5.5 \\
3.7-6.3 \\
\end{array}$ & $\begin{array}{c}16-31 \\
0-18 \\
\end{array}$ & $\begin{array}{c}0-5 \\
0-89 \\
\end{array}$ & $\begin{array}{c}73-90 \\
50-130 \\
\end{array}$ & $\begin{array}{c}79-92 \\
100-130 \\
\end{array}$ & $\begin{array}{l}\geq 3 \\
\geq 3\end{array}$ & $\begin{array}{l}\mathrm{LOD}_{95} \\
\mathrm{LOD}_{95}\end{array}$ & $\begin{array}{l}0.44 \\
0.28 \\
\end{array}$ & $\begin{array}{l}\mathrm{NR} \\
\mathrm{NR}\end{array}$ & $\begin{array}{l}0.267 \\
0.067\end{array}$ \\
\hline Brown 2007c & 8 & NR & NA & NR & NR & NA & $16.4-36.1$ & NA & NR & $28-90$ & NA & $\geq 1 \mathrm{CFU} / \mathrm{ml}$ & NR & NR & NR & $\mathrm{NR}$ \\
\hline Einfeld 2011 & 6 & NR & NA & NR & NR & NA & $10.0-19.7$ & NA & NR & $50-129$ & NA & NR & NR & NR & NR & NR \\
\hline Quizon 2007 & 6 & $890-5200$ & NA & $9-20^{(\mathrm{a})}$ & (a) & NA & $4.4, \mathrm{NR}$ & NA & NR & NR & NA & NR & NR & NR & NR & NR \\
\hline Montgomery 2008 & $\begin{array}{l}1 \\
4 \\
3 \\
2\end{array}$ & $\begin{array}{l}\text { NR } \\
\text { NR } \\
\text { NR } \\
\text { NR }\end{array}$ & $\begin{array}{l}\text { NA } \\
\text { NA } \\
\text { NA } \\
\text { NA }\end{array}$ & $\begin{array}{l}\text { NR } \\
\text { NR } \\
\text { NR } \\
\text { NR }\end{array}$ & $\begin{array}{l}\text { NR } \\
\text { NR } \\
\text { NR } \\
\text { NR }\end{array}$ & $\begin{array}{l}\text { NA } \\
\text { NA } \\
\text { NA } \\
\text { NA }\end{array}$ & $\begin{array}{c}2.42 \\
1.16-3.52 \\
0.20-0.48 \\
2.26,1.69\end{array}$ & $\begin{array}{l}\text { NA } \\
\text { NA } \\
\text { NA } \\
\text { NA }\end{array}$ & $\begin{array}{c}1.3 \\
16-28 \\
\text { NA } \\
\text { NA }\end{array}$ & $\begin{array}{c}24 \\
13-25 \\
10-60 \\
18,32\end{array}$ & $\begin{array}{l}\text { NA } \\
\text { NA } \\
\text { NA } \\
\text { NA }\end{array}$ & $\begin{array}{l}\text { NR } \\
\text { NR } \\
\text { NR } \\
\text { NR }\end{array}$ & $\begin{array}{l}\text { NR } \\
\text { NR } \\
\text { NR } \\
\text { NR }\end{array}$ & $\begin{array}{l}\text { NR } \\
\text { NR } \\
\text { NR } \\
\text { NR }\end{array}$ & $\begin{array}{l}\text { NR } \\
\text { NR } \\
\text { NR } \\
\text { NR }\end{array}$ & $\begin{array}{l}\text { NR } \\
\text { NR } \\
\text { NR } \\
\text { NR }\end{array}$ \\
\hline
\end{tabular}

(a) Combined estimate of "run" and "sample-within-run" uncertainties. 
Table 5a. Summary of test conditions for storage and stability tests. Acronyms and abbreviations are defined in Table 6.

\begin{tabular}{|c|c|c|c|c|c|c|c|c|c|c|c|c|c|c|c|c|c|}
\hline \multirow{2}{*}{$\begin{array}{l}\text { Reference } \\
\text { (lead author } \\
\text { and year) }\end{array}$} & \multirow[b]{2}{*}{$\begin{array}{c}\# \\
\text { tests }\end{array}$} & \multirow[b]{2}{*}{ Agent (a) } & \multirow{2}{*}{$\begin{array}{c}\text { Agent } \\
\text { deposition } \\
\text { (b) }\end{array}$} & \multirow{2}{*}{$\begin{array}{l}\text { Agent } \\
\text { concen- } \\
\text { tration } \\
(\mathrm{CFU} / \mathrm{ml})\end{array}$} & \multirow{2}{*}{$\begin{array}{l}\text { Sampling } \\
\text { medium } \\
\text { type (b) }\end{array}$} & \multirow{2}{*}{$\begin{array}{l}\text { Wetting } \\
\text { agent } \\
\text { (b) }\end{array}$} & \multirow{2}{*}{$\begin{array}{l}\text { Relative } \\
\text { humidity }\end{array}$} & \multirow{2}{*}{$\begin{array}{c}\text { Surface } \\
\text { type \& area } \\
\text { sampled (b) }\end{array}$} & \multicolumn{3}{|c|}{ Storage conditions } & \multirow{2}{*}{$\begin{array}{l}\text { Extract- } \\
\text { ion } \\
\text { liquid } \\
\text { (a) }\end{array}$} & \multirow{2}{*}{$\begin{array}{l}\text { Extract- } \\
\text { ion } \\
\text { method } \\
\text { (a) }\end{array}$} & \multirow{2}{*}{$\begin{array}{l}\text { Culture } \\
\text { method, } \\
\text { medium }\end{array}$} & \multirow[b]{2}{*}{$\begin{array}{c}\# \\
\text { labs }\end{array}$} & \multirow[b]{2}{*}{$\begin{array}{l}\# \text { test } \\
\text { runs }\end{array}$} & \multirow{2}{*}{$\begin{array}{c}\text { Total } \\
\text { \# test } \\
\text { samples }\end{array}$} \\
\hline & & & & & & & & & $\begin{array}{l}\text { Additive } \\
\text { (c) }\end{array}$ & $\begin{array}{l}\text { Temp. } \\
\left({ }^{\circ} \mathrm{C}\right)\end{array}$ & $\begin{array}{c}\# \\
\text { days }\end{array}$ & & & & & & \\
\hline \multirow{3}{*}{$\begin{array}{l}\text { Almeida } \\
2008\end{array}$} & 1 & BA Sterne & Liquid & NR & NA & NA & $\mathrm{NR}$ & NA & None & 4 & 0 & NA & NA & Plate, LBA & NR & 3 lots & 19 \\
\hline & 15 & BA Sterne & Liquid & NR & NA & NA & NR & NA & $\begin{array}{l}\text { None, } \\
\text { Phenol, } \\
\text { EDTA. } \\
\text { Ethanol, } \\
\text { PBSTr }\end{array}$ & 4 & $\begin{array}{c}0, \\
182, \\
279\end{array}$ & NA & NA & Plate, LBA & NR & 1 & 3 \\
\hline & 4 & BA Sterne & Liquid & NR & NA & NA & NR & NA & None & $\begin{array}{l}-20 \\
-80\end{array}$ & $\begin{array}{l}182, \\
279\end{array}$ & NA & NA & Plate, LBA & NR & 1 & 3 \\
\hline
\end{tabular}

(a) The BA Sterne spores were diluted with PBS (10 mmol/L phosphate, $138 \mathrm{mmol} / \mathrm{L} \mathrm{NaCl}$, and $2.7 \mathrm{mmol} / \mathrm{L} \mathrm{KCl}$, $\mathrm{pH} 7.4)$ containing $0.01 \%$ Triton ${ }^{\circledR} \mathrm{X}-100$, and vigorously mixed by vortexing.

(b) This study used only liquid samples containing the agent, so neither deposition onto surfaces nor sampling of surfaces was involved.

(c) Additives to sterile water: Ethanol = ethanol $20 \%(\mathrm{v} / \mathrm{v})$, EDTA = ethylendediaminetetraacetic acid, $10 \mathrm{mmol} / \mathrm{L}, \mathrm{pH} 8.0, \mathrm{Phenol}=\mathrm{phenol} 1 \%(\mathrm{v} / \mathrm{v})$, PBSTr $=\mathrm{PBS}$ containing

$0.01 \%(\mathrm{v} / \mathrm{v})$ Triton $\times 100$.

Table 5b. Summary of results for storage and stability tests. Acronyms and abbreviations are defined in Table 6 .

\begin{tabular}{|c|c|c|c|c|c|c|c|c|c|c|c|c|c|c|c|c|}
\hline \multirow[b]{2}{*}{$\begin{array}{l}\text { Reference } \\
\text { (lead author } \\
\text { and year) }\end{array}$} & \multirow[b]{2}{*}{$\begin{array}{c}\# \\
\text { tests }\end{array}$} & \multicolumn{5}{|c|}{$\begin{array}{c}\text { Recovery concentration results - } \\
\text { Mean \& \%RSDs }\end{array}$} & \multicolumn{5}{|c|}{$\begin{array}{l}\text { Recovery Efficiency }(\mathrm{RE})- \\
\text { Mean \& \% RSDs } \\
\end{array}$} & \multicolumn{5}{|c|}{ LOD, FNR, and FPR } \\
\hline & & $\begin{array}{c}\text { Mean } \\
(\mathrm{CFU} / \mathrm{ml})\end{array}$ & $\begin{array}{l}\text { Lab } \\
\% \text { RSD }\end{array}$ & $\begin{array}{l}\text { Run } \\
\% \text { RSD }\end{array}$ & $\begin{array}{c}\text { Sample- } \\
\text { within- } \\
\text { run } \% \text { RSD }\end{array}$ & $\begin{array}{l}\text { Total } \\
\% \text { RSD }\end{array}$ & $\begin{array}{c}\mathrm{RE} \\
\text { mean } \\
(\%) \\
\end{array}$ & $\begin{array}{l}\text { RE lab } \\
\% \text { RSD }\end{array}$ & $\begin{array}{l}\text { RE run } \\
\% \text { RSD }\end{array}$ & $\begin{array}{c}\text { RE sample- } \\
\text { within-run } \\
\% \text { RSD } \\
\end{array}$ & $\begin{array}{c}\mathrm{RE} \\
\text { total } \\
\% \mathrm{RSD} \\
\end{array}$ & $\begin{array}{c}\text { Positive } \\
\text { result } \\
(\mathrm{CFU})\end{array}$ & $\begin{array}{l}\text { LOD } \\
\text { defi- } \\
\text { nition } \\
\end{array}$ & $\begin{array}{c}\text { LOD } \\
\left(\mathrm{CFU} / \mathrm{cm}^{2}\right)\end{array}$ & FNR & FPR \\
\hline Almeida 2008 & $\begin{array}{c}1 \\
15 \\
4\end{array}$ & $\begin{array}{c}6.19 \mathrm{E}+9 \\
1.00 \mathrm{E}+8-1.95 \mathrm{E}+8 \\
1.20 \mathrm{E}+8-1.61 \mathrm{E}+8\end{array}$ & $\begin{array}{l}\text { NA } \\
\text { NA } \\
\text { NA }\end{array}$ & $\begin{array}{l}174 \\
\text { NA } \\
\text { NA }\end{array}$ & $\begin{array}{c}25 \\
4-86 \\
7-84\end{array}$ & $\begin{array}{l}\text { NA } \\
\text { NA } \\
\text { NA }\end{array}$ & $\begin{array}{c}111 \\
71-86 \\
\text { NA }\end{array}$ & $\begin{array}{l}\text { NA } \\
\text { NA } \\
\text { NA }\end{array}$ & $\begin{array}{c}22 \\
22 \\
6-55\end{array}$ & $\begin{array}{r}24 \\
24 \\
\text { NA }\end{array}$ & $\begin{array}{l}\text { NA } \\
\text { NA } \\
\text { NA }\end{array}$ & $\begin{array}{l}\mathrm{NR} \\
\mathrm{NR} \\
\mathrm{NR}\end{array}$ & $\begin{array}{l}\mathrm{NR} \\
\mathrm{NR} \\
\mathrm{NR}\end{array}$ & $\begin{array}{l}\text { NR } \\
\text { NR } \\
\text { NR }\end{array}$ & $\begin{array}{l}\text { NR } \\
\text { NR } \\
\text { NR }\end{array}$ & $\begin{array}{l}\text { NR } \\
\text { NR } \\
\text { NR }\end{array}$ \\
\hline
\end{tabular}

(a) Combined estimate of "run" and "sample-within-run" uncertainties. 
Table 6. List of acronyms and abbreviations

\begin{tabular}{|c|c|}
\hline A & agitation (during processing/extraction) \\
\hline Alum & aluminum \\
\hline ATD & Arizona Test Dust \\
\hline B & brick \\
\hline BA & Bacillus anthracis \\
\hline BA (4) & four strains of Bacillus anthracis \\
\hline BA-S & Bacillus anthracis Sterne \\
\hline BAtr & Bacillus atrophaeus \\
\hline $\begin{array}{l}\text { BAtr + } \\
\text { grime }\end{array}$ & $\begin{array}{l}\text { Bacillus atrophaeus + "standard grime" } \\
\text { (Arizona test dust }+ \text { diesel carbon }+ \text { oil }+ \\
\text { biologicals) }\end{array}$ \\
\hline BB & Butterfield buffer \\
\hline BBT & Butterfield buffer with Tween \\
\hline BHIA & brain heart infusion agar \\
\hline BiSKit $^{\mathrm{TM}}$ & Biological Sampling Kit \\
\hline BS & Bacillus subtilis \\
\hline $\mathrm{BS}+\mathrm{PC} 2$ & Bacillus subtilis with $10^{2}$ or $10^{4} \mathrm{CFU} / \mathrm{cm}^{2} P$. \\
\hline $\mathrm{BS}+\mathrm{PC} 4$ & chrysogenum background contamination \\
\hline $\mathrm{C}$ & centrifuging (during processing/extraction) \\
\hline CelT & ceiling tile \\
\hline CerT & ceramic tile \\
\hline CFU & colony forming unit \\
\hline CI & confidence interval \\
\hline Cpt & carpet \\
\hline Cpt-C & carpet, commercial loop \\
\hline Cpt-R & carpet, residential cut-pile \\
\hline Cpt-S & carpet, soiled residential cut-pile \\
\hline $\operatorname{Cpt}(3)$ & Cpt-C, Cpt-R, Cpt-S \\
\hline $\mathrm{Ct}$ & cotton \\
\hline Ct cloth & cotton cloth \\
\hline DA & dry aerosol \\
\hline DE broth & Dey Engley broth \\
\hline DI & directly inoculated \\
\hline DP & Dacron $^{\circledR}$ polyester \\
\hline DW & de-ionized, distilled, or sterile water \\
\hline EWD & drops of agent in $50 \%$ ethanol, $50 \%$ water \\
\hline $\mathrm{EtOH}$ & Ethyl alcohol, enthanol \\
\hline $\mathrm{F}$ & Formica $^{\circledR}$ \\
\hline FL & faux leather \\
\hline FNR & false negative rate \\
\hline FP & filter plate \\
\hline FPR & false positive rate \\
\hline G & glass \\
\hline $\mathrm{H}$ & heat treatment (extraction/processing) \\
\hline HCW & HS II Cleanroom Wiper \\
\hline HEPA & high efficiency particulate air \\
\hline $\mathrm{HM}$ & hand mixed \\
\hline HS & hand stomached \\
\hline HVAC & heating, ventilation, and air conditioning \\
\hline HW & heavy wipe \\
\hline LBA & luria broth agar \\
\hline LD & liquid drops \\
\hline LI & liquid immersion \\
\hline LOD & limit of detection \\
\hline $\mathrm{LOD}_{90}$ & lowest concentration with $90 \%$ or $95 \%$ \\
\hline $\mathrm{LOD}_{95}$ & probability of detection \\
\hline LS & liquid spread \\
\hline M & metal \\
\hline MF, Mfoam & macrofoam \\
\hline MLI foil & multilayer insulation foil (aluminized Kapton) \\
\hline
\end{tabular}

\begin{tabular}{|c|c|}
\hline NA & not applicable \\
\hline NB & neutralizing buffer \\
\hline $\mathrm{NB}+\mathrm{ATD}$ & $\begin{array}{l}\text { sponge wipe pre-moistened with neutralizing } \\
\text { buffer, wipe samples collected, then inoculated } \\
\text { with Arizona Test Dust }\end{array}$ \\
\hline NR & not reported \\
\hline $\mathrm{O}$ & oak wood \\
\hline $\mathrm{P}$ & Plastic \\
\hline P2D & Pulled one direction \& perpendicular direction \\
\hline PBS & phosphate buffered saline \\
\hline PBST & PBS with Tween \\
\hline PBST + & $\begin{array}{l}\text { PBST }+ \text { Arizona Test Dust }+ \text { Bacillus } \\
\text { atrophaeus }+ \text { Staphylococcus epidermidis }\end{array}$ \\
\hline PBSTr & PBS-TritonX-100 \\
\hline $\mathrm{PBST} / \mathrm{Tr}$ & $\begin{array}{l}\text { PBS with } 0.1 \% \text { Tween (PBST) or } 0.1 \% \\
\text { Triton-X (PBSTr) }\end{array}$ \\
\hline PBT & potassium phosphate buffer w/ Tween \\
\hline PC & Polycarbonate \\
\hline PD & petri dish \\
\hline $\mathrm{PE}$ & polyester \\
\hline PE filter & polyethylene filter \\
\hline PEF & polyester foam \\
\hline PEUF & polyester upholstery fabric \\
\hline $\mathrm{PF}$ & Petrifilm ${ }^{\mathrm{TM}}$ \\
\hline PLCP & plastic light cover panel \\
\hline PP2D & push-pull one direction, then other \\
\hline PR & polyester-rayon blend \\
\hline PR gauze & polyester-rayon blend gauze \\
\hline PU foam & polyurethane foam \\
\hline PW & painted wood \\
\hline PWB & painted wallboard \\
\hline $\mathrm{R}$ & Rayon \\
\hline RE & recovery efficiency \\
\hline RH & relative humidity \\
\hline$\%$ RSD & percent relative standard deviation \\
\hline S & Sonication \\
\hline SBA & sheep blood agar \\
\hline SD & standard deviation \\
\hline SE & standard error \\
\hline SK & swab kit \\
\hline SP & spread plate \\
\hline Sp. stick & sponge stick \\
\hline Sp. wipe & sponge wipe \\
\hline $\mathrm{SP} / \mathrm{FP}$ & $\begin{array}{l}\text { spread plate for higher concentrations, filter } \\
\text { plate for lower concentrations }\end{array}$ \\
\hline SprP & spiral plate \\
\hline SS & stainless steel \\
\hline SSPK & swab sample processing kit \\
\hline St & Stomached \\
\hline SVH & S strokes, vertical then horizontal \\
\hline TECF & 3M Trace Evidence Collection Filter \\
\hline TSA & trypticase soy agar \\
\hline TSAB & $\begin{array}{l}\text { trypticase soy agar with } 5 \% \text { sheep blood (also } \\
\text { referred to as TSAII) }\end{array}$ \\
\hline TSAC & trypticase soy agar $+100 \mu \mathrm{g}$ cycloheximide $/ \mathrm{ml}$ \\
\hline $\mathrm{U}$ & Untreated \\
\hline V & vortexing (during processing/extraction) \\
\hline VL & vinyl or vinyl tile \\
\hline W & Wood \\
\hline WA & wet aerosol \\
\hline WD & drops of agent dispersed in water \\
\hline
\end{tabular}


Table 7. Numbers of the 20 surface sampling studies that investigated various factors and reported specific results

\begin{tabular}{|c|c|c|c|c|c|c|c|c|c|c|c|c|c|c|c|c|c|c|}
\hline \multirow[b]{3}{*}{$\begin{array}{l}\text { Number of Studies that } \\
\text { Varied the ... }\end{array}$} & \multicolumn{6}{|c|}{ Swabs (13) $^{(\mathrm{a})}$} & \multicolumn{6}{|c|}{ Wipes (12) $^{(\mathbf{a})}$} & \multicolumn{6}{|c|}{ Vacuums (5) ${ }^{(\mathbf{a})}$} \\
\hline & \multicolumn{2}{|c|}{$\operatorname{Rec}^{(\mathrm{b})}$} & \multicolumn{2}{|c|}{$\mathrm{RE}^{(\mathrm{c})}$} & \multirow[b]{2}{*}{$\stackrel{E}{\stackrel{E}{\circ}}$} & \multirow[b]{2}{*}{ 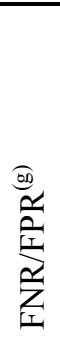 } & \multicolumn{4}{|c|}{$\operatorname{Rec}^{(\mathrm{b})} \quad \mathrm{RE}^{(\mathrm{c})}$} & \multirow[b]{2}{*}{ Eิ } & \multirow[b]{2}{*}{ 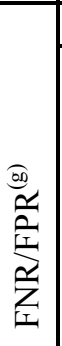 } & \multicolumn{4}{|c|}{$\operatorname{Rec}^{(\mathrm{b})} \quad \mathrm{RE}^{(\mathrm{c})}$} & \multirow[b]{2}{*}{$\stackrel{\Xi}{\stackrel{\Xi}{\circ}}$} & \multirow[b]{2}{*}{ 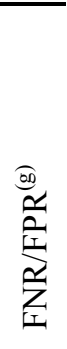 } \\
\hline & 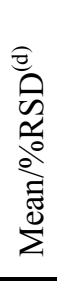 & 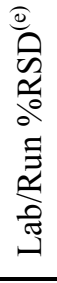 & 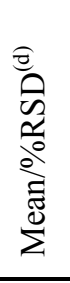 & 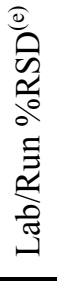 & & & 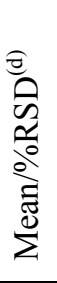 & 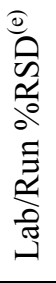 & 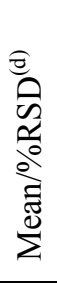 & 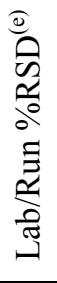 & & & 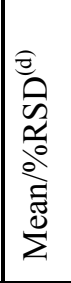 & 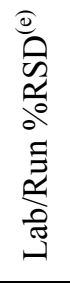 & 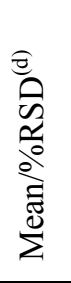 & 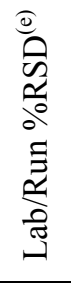 & & \\
\hline Agent (contaminant) & 1 & 0 & 1 & 0 & 0 & 0 & 1 & 0 & 1 & 0 & 0 & 0 & 0 & 0 & 1 & 0 & 0 & 0 \\
\hline & 1 & 0 & 4 & 1 & 0 & 1 & 1 & 0 & 0 & 0 & 0 & 0 & 0 & 0 & 1 & 0 & 0 & 0 \\
\hline & 5 & 1 & 9 & 2 & 3 & & 4 & 1 & 5 & 2 & 5 & 3 & 1 & 1 & 3 & 1 & 1 & 1 \\
\hline & 3 & 0 & 5 & 0 & 0 & 0 & 1 & 0 & 3 & 0 & 0 & 0 & 0 & 0 & 0 & 0 & 0 & 0 \\
\hline Wett & 1 & 0 & 3 & 1 & 0 & 1 & 1 & 0 & 3 & 0 & 1 & 0 & NA & NA & NA & NA & NA & NA \\
\hline Surfac & 5 & 1 & 4 & 1 & 1 & 1 & 6 & 1 & 7 & 1 & 3 & 2 & 2 & 1 & 5 & 1 & 1 & 1 \\
\hline Surfac & 1 & 0 & 1 & 0 & 0 & 0 & 0 & 0 & 1 & 0 & 0 & 0 & 0 & 0 & 1 & 0 & 0 & 0 \\
\hline Jtronsnort conditions (h) & 0 & 0 & 0 & 0 & 0 & 0 & 0 & 0 & 0 & 0 & 0 & 0 & 0 & 0 & 0 & 0 & 0 & 0 \\
\hline Processing/extraction method & 4 & 0 & 4 & 0 & 1 & 0 & 0 & 0 & 0 & 0 & 0 & 0 & 0 & 0 & 0 & 0 & 0 & 0 \\
\hline Number of labs & 3 & 1 & 3 & 2 & 2 & 3 & 2 & 1 & 2 & 2 & 2 & 2 & 0 & 0 & 0 & 0 & 0 & 0 \\
\hline
\end{tabular}
(a) Number in parentheses is the number of studies summarized.
(b) "Rec" denotes the agent recovered by surface sampling.
(c) RE denotes recovery efficiency.
(d) Mean $/ \% \mathrm{RSD}$ denotes the mean and within-run \%RSD.
(e) Lab/Run \%RSD means lab-to-lab \%RSD and run-to-run \%RSD.
(f) LOD denotes limit of detection
(g) FNR/FPR denotes false negative and/or false positive rate.
(h) Storage results are available in Almeida et al. (2008), but sampling media were not part of that study. 


\section{APPENDIX \\ Detailed Summary Tables of Test Conditions and Results from Laboratory Studies of Swab, Wipe, and Vacuum Sampling of Surfaces Contaminated with Bacillus anthracis or Surrogates}

Tables A.1, A.2, and A.3 summarize the characteristics and results of laboratory studies from the literature (individual tests or groups of tests conducted in a laboratory or chamber) to investigate the performance of swab, wipe, and vacuum surface sampling and analysis methods when 1) the surfaces are contaminated with Bacillus anthracis or surrogates, and 2) culturing/counting is used to quantify results. Table A.4 summarizes the characteristics and results of one study (which did not involve surface sampling) to investigate the effects of storage/transportation factors. The contents of Tables A.1 to A.4 are explained in the section "Summary of Anthrax-Related Laboratory Studies." Also, the column headings of Tables A.1 to A.4 are described in Table 1, while the acronyms and abbreviations used in the tables are defined in Table 6. 
Table A.1a Expanded summary of test conditions for swab sampling. Acronyms and abbreviations are defined in Table 6.

\begin{tabular}{|c|c|c|c|c|c|c|c|c|c|c|c|c|c|c|c|}
\hline Reference & $\begin{array}{c}\text { Test } \\
\# \\
\end{array}$ & Agent & $\begin{array}{c}\text { Agent } \\
\text { deposition }\end{array}$ & $\begin{array}{c}\text { Agent } \\
\text { concentration } \\
\left(\mathrm{CFU} / \mathrm{cm}^{2}\right) \\
\end{array}$ & $\begin{array}{l}\text { Swab } \\
\text { type }\end{array}$ & $\begin{array}{c}\text { Wetting } \\
\text { agent }\end{array}$ & $\begin{array}{c}\text { Relative } \\
\text { humidity } \\
(\%)\end{array}$ & $\begin{array}{c}\text { Surface } \\
\text { type }\end{array}$ & $\begin{array}{c}\text { Surface } \\
\text { area } \\
\text { sampled } \\
\left(\mathrm{cm}^{2}\right) \\
\end{array}$ & $\begin{array}{c}\text { Extraction } \\
\text { liquid }\end{array}$ & $\begin{array}{l}\text { Extraction } \\
\text { method }\end{array}$ & $\begin{array}{l}\text { Culture } \\
\text { method, } \\
\text { medium }\end{array}$ & $\begin{array}{c}\# \\
\text { labs }\end{array}$ & $\begin{array}{c}\# \text { test } \\
\text { runs }\end{array}$ & $\begin{array}{c}\text { Total } \\
\text { \# test } \\
\text { samples } \\
\end{array}$ \\
\hline Estill et & 1.1 & BA Sterne & Dry aerosol & 0.03 & Macrofoam & BBT & NR & $\mathrm{SS}$ & 103 & BBT & $\mathrm{V}+\mathrm{S}$ & FP, TSAB & 3 & 3 & 36 \\
\hline \multirow[t]{5}{*}{ al. 2009} & 1.2 & BA Sterne & Dry aerosol & 0.3 & Macrofoam & BBT & NR & SS & 103 & BBT & $\mathrm{V}+\mathrm{S}$ & FP, TSAB & 3 & 3 & 36 \\
\hline & 1.3 & BA Sterne & Dry aerosol & 2 & Macrofoam & BBT & NR & SS & 103 & BBT & $\mathrm{V}+\mathrm{S}$ & FP, TSAB & 3 & 3 & 35 \\
\hline & 1.4 & BA Sterne & Dry aerosol & 0.03 & Macrofoam & BBT & NR & Carpet & 103 & $\mathrm{BBT}$ & $\mathrm{V}+\mathrm{S}$ & FP, TSAB & 3 & 2 & 24 \\
\hline & 1.5 & BA Sterne & Dry aerosol & 0.3 & Macrofoam & BBT & NR & Carpet & 103 & BBT & $\mathrm{V}+\mathrm{S}$ & $\mathrm{FP}, \mathrm{TSAB}$ & 3 & 4 & 48 \\
\hline & 1.6 & BA Sterne & Dry aerosol & 2 & Macrofoam & BBT & NR & Carpet & 103 & BBT & $\mathrm{V}+\mathrm{S}$ & FP, TSAB & 3 & 3 & 36 \\
\hline Hodges et & 2.1 & BA Sterne & Liquid drops & 0.4 & Macrofoam & PBST & NR & SS & 10 & PBST & $\mathrm{V}$ & Plate, TSAB & $2^{(\mathrm{a})}$ & NR & 15 \\
\hline \multirow[t]{5}{*}{ al. 2006} & 2.2 & BA Sterne & Liquid drops & 0.6 & Macrofoam & PBST & NR & $\mathrm{SS}$ & 10 & PBST & $\mathrm{V}$ & Plate, TSAB & $2^{(\mathrm{a})}$ & NR & 15 \\
\hline & 2.3 & BA Sterne & Liquid drops & 1.2 & Macrofoam & PBST & NR & SS & 10 & PBST & V & Plate, TSAB & $2^{\text {(a) }}$ & NR & 15 \\
\hline & 2.4 & BA Sterne & Liquid drops & 3.8 & Macrofoam & PBST & NR & SS & 10 & PBST & $\mathrm{V}$ & Plate, TSAB & $2^{(\mathrm{a})}$ & NR & 15 \\
\hline & 2.5 & BA Sterne & Liquid drops & 59 & Macrofoam & PBST & NR & SS & 10 & PBST & $\mathrm{V}$ & Plate, TSAB & $2^{(\mathrm{a})}$ & NR & 45 \\
\hline & 2.6 & BA Sterne & Liquid drops & 6000 & Macrofoam & PBST & NR & $\mathrm{SS}$ & 10 & PBST & $\mathrm{V}$ & FP, TSAB & $2^{(\mathrm{a})}$ & NR & 45 \\
\hline Rose et & 3.1 & BA Sterne & Liquid drops & 1937.5 & Cotton & Dry & NR & $\mathrm{SS}$ & 25.8 & PBST & None & Plate, TSAB & 1 & NR & 10 \\
\hline \multirow[t]{27}{*}{ al. 2004} & 3.2 & BA Sterne & Liquid drops & 1937.5 & Cotton & PBST & NR & SS & 25.8 & PBST & None & Plate, TSAB & 1 & NR & 10 \\
\hline & 3.3 & BA Sterne & Liquid drops & 1937.5 & Cotton & Dry & NR & SS & 25.8 & PBST & V & Plate, TSAB & 1 & NR & 10 \\
\hline & 3.4 & BA Sterne & Liquid drops & 1937.5 & Cotton & PBST & NR & SS & 25.8 & PBST & $\mathrm{V}$ & Plate, TSAB & 1 & NR & 10 \\
\hline & 3.5 & BA Sterne & Liquid drops & 1937.5 & Cotton & Dry & NR & SS & 25.8 & PBST & $\mathrm{S}$ & Plate, TSAB & 1 & NR & 10 \\
\hline & 3.6 & BA Sterne & Liquid drops & 1937.5 & Cotton & PBST & NR & SS & 25.8 & PBST & $\mathrm{S}$ & Plate, TSAB & 1 & NR & 10 \\
\hline & 3.7 & BA Sterne & Liquid drops & 1937.5 & Polyester & Dry & NR & SS & 25.8 & PBST & None & Plate, TSAB & 1 & NR & 10 \\
\hline & 3.8 & BA Sterne & Liquid drops & 1937.5 & Polyester & PBST & NR & $\mathrm{SS}$ & 25.8 & PBST & None & Plate, TSAB & 1 & NR & 10 \\
\hline & 3.9 & BA Sterne & Liquid drops & 1937.5 & Polyester & Dry & NR & SS & 25.8 & PBST & $\mathrm{V}$ & Plate, TSAB & 1 & NR & 10 \\
\hline & 3.10 & BA Sterne & Liquid drops & 1937.5 & Polyester & PBST & NR & SS & 25.8 & PBST & V & Plate, TSAB & 1 & NR & 10 \\
\hline & 3.11 & BA Sterne & Liquid drops & 1937.5 & Polyester & Dry & NR & $\mathrm{SS}$ & 25.8 & PBST & $\mathrm{S}$ & Plate, TSAB & 1 & NR & 10 \\
\hline & 3.12 & BA Sterne & Liquid drops & 1937.5 & Polyester & PBST & NR & SS & 25.8 & PBST & $\mathrm{S}$ & Plate, TSAB & 1 & NR & 10 \\
\hline & 3.13 & BA Sterne & Liquid drops & 1937.5 & Rayon & Dry & NR & SS & 25.8 & PBST & None & Plate, TSAB & 1 & NR & 10 \\
\hline & 3.14 & BA Sterne & Liquid drops & 1937.5 & Rayon & PBST & NR & $\mathrm{SS}$ & 25.8 & PBST & None & Plate, TSAB & 1 & NR & 10 \\
\hline & 3.15 & BA Sterne & Liquid drops & 1937.5 & Rayon & Dry & NR & SS & 25.8 & PBST & $\mathrm{V}$ & Plate, TSAB & 1 & NR & 10 \\
\hline & 3.16 & BA Sterne & Liquid drops & 1937.5 & Rayon & PBST & NR & SS & 25.8 & PBST & $\mathrm{V}$ & Plate, TSAB & 1 & NR & 10 \\
\hline & 3.17 & BA Sterne & Liquid drops & 1937.5 & Rayon & Dry & NR & $\mathrm{SS}$ & 25.8 & PBST & $\mathrm{S}$ & Plate, TSAB & 1 & NR & 10 \\
\hline & 3.18 & BA Sterne & Liquid drops & 1937.5 & Rayon & PBST & NR & SS & 25.8 & PBST & S & Plate, TSAB & 1 & NR & 10 \\
\hline & 3.19 & BA Sterne & Liquid drops & 1937.5 & Macrofoam & Dry & NR & SS & 25.8 & PBST & None & Plate, TSAB & 1 & NR & 10 \\
\hline & 3.20 & BA Sterne & Liquid drops & 1937.5 & Macrofoam & PBST & NR & SS & 25.8 & PBST & None & Plate, TSAB & 1 & NR & 10 \\
\hline & 3.21 & BA Sterne & Liquid drops & 1937.5 & Macrofoam & Dry & NR & $\mathrm{SS}$ & 25.8 & PBST & V & Plate, TSAB & 1 & NR & 10 \\
\hline & 3.22 & BA Sterne & Liquid drops & 1937.5 & Macrofoam & PBST & NR & $\mathrm{SS}$ & 25.8 & PBST & $\mathrm{V}$ & Plate, TSAB & 1 & NR & 10 \\
\hline & 3.23 & BA Sterne & Liquid drops & 1937.5 & Macrofoam & Dry & NR & SS & 25.8 & PBST & $\mathrm{S}$ & Plate, TSAB & 1 & NR & 10 \\
\hline & 3.24 & BA Sterne & Liquid drops & 1937.5 & Macrofoam & PBST & NR & SS & 25.8 & PBST & $\mathrm{S}$ & Plate, TSAB & 1 & NR & 10 \\
\hline & 3.25 & BA Sterne & Liquid imm. & $1 \mathrm{E}+4 \mathrm{CFU} / \mathrm{swab}$ & Cotton, DI & NA & NR & None & NA & PBST & $\mathrm{V}$ & Plate, TSAB & 1 & NR & 10 \\
\hline & 3.26 & BA Sterne & Liquid imm. & $1 \mathrm{E}+4 \mathrm{CFU} / \mathrm{swab}$ & Polyester & NR & NR & None & NA & PBST & $\mathrm{V}$ & Plate, TSAB & 1 & NR & 10 \\
\hline & 3.27 & BA Sterne & Liquid imm. & $1 \mathrm{E}+4 \mathrm{CFU} / \mathrm{swab}$ & Rayon & NR & NR & None & NA & PBST & $\mathrm{V}$ & Plate, TSAB & 1 & NR & 10 \\
\hline & 3.28 & BA Sterne & Liquid imm. & $1 \mathrm{E}+4 \mathrm{CFU} / \mathrm{swab}$ & Macrofoam & NR & NR & None & NA & PBST & V & Plate, TSAB & 1 & NR & 10 \\
\hline
\end{tabular}


Table A.1a Expanded summary of test conditions for swab sampling. Acronyms and abbreviations are defined in Table 6. (cont.)

\begin{tabular}{|c|c|c|c|c|c|c|c|c|c|c|c|c|c|c|c|}
\hline Reference & $\begin{array}{c}\text { Test } \\
\# \\
\end{array}$ & Agent & $\begin{array}{c}\text { Agent } \\
\text { deposition }\end{array}$ & $\begin{array}{c}\text { Agent } \\
\text { concentration } \\
\left(\mathrm{CFU} / \mathrm{cm}^{2}\right)\end{array}$ & $\begin{array}{l}\text { Swab } \\
\text { type }\end{array}$ & $\begin{array}{c}\text { Wetting } \\
\text { agent }\end{array}$ & $\begin{array}{c}\text { Relative } \\
\text { humidity } \\
(\%)\end{array}$ & $\begin{array}{c}\text { Surface } \\
\text { type }\end{array}$ & $\begin{array}{l}\text { Surface } \\
\text { area } \\
\text { sampled } \\
\left(\mathrm{cm}^{2}\right) \\
\end{array}$ & $\begin{array}{c}\text { Extraction } \\
\text { liquid }\end{array}$ & $\begin{array}{l}\text { Extraction } \\
\text { method }\end{array}$ & $\begin{array}{l}\text { Culture } \\
\text { method, } \\
\text { medium }\end{array}$ & $\begin{array}{c}\# \\
\text { labs }\end{array}$ & $\begin{array}{l}\# \text { test } \\
\text { runs }\end{array}$ & $\begin{array}{c}\text { Total } \\
\text { \# test } \\
\text { samples } \\
\end{array}$ \\
\hline \multirow{13}{*}{$\begin{array}{l}\text { Frawley } \\
\text { et al. } 2008\end{array}$} & 4.1 & BA Sterne & Liquid drops & $1 \mathrm{E}+2-1 \mathrm{E}+5$ & Polyester & Dry & NR & Plastic & 1 & PBST & $\mathrm{V}$ & Plate, SBA & 1 & NR & 12 \\
\hline & 4.2 & BA Sterne & Liquid drops & (b) & Polyester & Dry & NR & Wood & NR & PBST & $\mathrm{V}$ & Plate, SBA & 1 & NR & 12 \\
\hline & 4.3 & BA Sterne & Liquid drops & (b) & Polyester & Dry & NR & Ct Cloth & NR & PBST & V & Plate, SBA & 1 & NR & 12 \\
\hline & 4.4 & BA Sterne & Liquid drops & $1 E+2-1 E+5$ & Polyester & $\mathrm{PBST} / \mathrm{Tr}$ & NR & Plastic & 1 & PBST & V & Plate, SBA & 1 & NR & 24 \\
\hline & 4.5 & BA Sterne & Liquid drops & (b) & Polyester & $\mathrm{PBST} / \mathrm{Tr}$ & NR & Wood & NR & PBST & V & Plate, SBA & 1 & NR & 24 \\
\hline & 4.6 & BA Sterne & Liquid drops & (b) & Polyester & $\mathrm{PBST} / \mathrm{Tr}$ & NR & Ct Cloth & NR & PBST & $\mathrm{V}$ & Plate, SBA & 1 & NR & 24 \\
\hline & 4.7 & $\mathrm{BA}(4)$ & Liquid drops & $50 \mathrm{CFU} /$ sample & Cotton & $\mathrm{PBST} / \mathrm{Tr}$ & NR & Plastic & NR & NR & NR & Plate, NR & 1 & NR & 11 \\
\hline & 4.8 & BA (4) & Liquid drops & $50 \mathrm{CFU} /$ sample & Cotton & $\mathrm{PBST} / \mathrm{Tr}$ & NR & Glass & NR & NR & NR & Plate, NR & 1 & NR & 11 \\
\hline & 4.9 & $\mathrm{BA}(4)$ & Liquid drops & $50 \mathrm{CFU} /$ sample & Cotton & $\mathrm{PBST} / \mathrm{Tr}$ & NR & Formica & NR & NR & NR & Plate, NR & 1 & NR & 11 \\
\hline & 4.10 & BA (4) & Liquid drops & $50 \mathrm{CFU} /$ sample & Cotton & $\mathrm{PBST} / \mathrm{Tr}$ & NR & Metal & NR & NR & NR & Plate, NR & 1 & NR & 11 \\
\hline & 4.11 & $\mathrm{BA}(4)$ & Liquid drops & $50 \mathrm{CFU} /$ sample & Cotton & $\mathrm{PBST} / \mathrm{Tr}$ & NR & Carpet & NR & NR & NR & Plate, NR & 1 & NR & 11 \\
\hline & 4.12 & BA (4) & Liquid drops & $50 \mathrm{CFU} /$ sample & Cotton & $\mathrm{PBST} / \mathrm{Tr}$ & NR & Brick & NR & NR & NR & Plate, NR & 1 & NR & 11 \\
\hline & 4.13 & BA, 1 strain & Liquid drops & $50 \mathrm{CFU} /$ sample & Cotton & $\mathrm{PBST} / \mathrm{Tr}$ & NR & Ct Cloth & NR & NR & NR & Plate, NR & 1 & NR & 11 \\
\hline \multirow{6}{*}{$\begin{array}{l}\text { Brown et } \\
\text { al. } 2007 b\end{array}$} & 5.1 & BAtr & Dry aerosol & $1 E+2-1 E+3$ & Rayon & DW & NR & SS & 25 & BBT & $\mathrm{S}+\mathrm{H}+\mathrm{V}$ & Plate, PF & 1 & NR & 20 \\
\hline & 5.2 & BAtr & Dry aerosol & $1 \mathrm{E}+4-1 \mathrm{E}+5$ & Rayon & DW & NR & SS & 25 & $\mathrm{BBT}$ & $\mathrm{S}+\mathrm{H}+\mathrm{V}$ & Plate, PF & 1 & NR & 20 \\
\hline & 5.3 & BAtr & Dry aerosol & $1 E+2-1 E+3$ & Rayon & DW & NR & PWB & 25 & BBT & $\mathrm{S}+\mathrm{H}+\mathrm{V}$ & Plate, PF & 1 & NR & 20 \\
\hline & 5.4 & BAtr & Dry aerosol & $1 \mathrm{E}+4-1 \mathrm{E}+5$ & Rayon & DW & NR & PWB & 25 & BBT & $\mathrm{S}+\mathrm{H}+\mathrm{V}$ & Plate, PF & 1 & NR & 20 \\
\hline & 5.5 & BAtr & Liquid imm. & $1 \mathrm{E}+6 \mathrm{CFU} / \mathrm{swab}$ & Rayon, DI & NA & NR & None & NA & BBT & $\mathrm{S}+\mathrm{H}+\mathrm{V}$ & Plate, $\mathrm{PF}$ & 1 & NR & 20 \\
\hline & 5.6 & BAtr & Liquid drops & $2.0 \mathrm{E}+5$ & NA & NA & NR & SS, DI & 6.25 & $\mathrm{BBT}$ & $\mathrm{S}+\mathrm{H}$ & Plate, BHIA & 1 & NR & 24 \\
\hline \multirow{20}{*}{$\begin{array}{l}\text { Edmonds } \\
\text { et al. } 2009\end{array}$} & 6.1 & BAtr & Liquid drops & $1.0 \mathrm{E}+5$ & Cotton & DW & NR & Glass & 10 & PBSTr & $\mathrm{V}+\mathrm{S}$ & SprP, NR & 1 & 4 & 40 \\
\hline & 6.2 & BAtr & Liquid drops & $1.0 \mathrm{E}+5$ & Dacron & DW & NR & Glass & 10 & PBSTr & $\mathrm{V}+\mathrm{S}$ & SprP, NR & 1 & 4 & 40 \\
\hline & 6.3 & BAtr & Liquid drops & $1.0 \mathrm{E}+5$ & Rayon & DW & NR & Glass & 10 & PBSTr & $\mathrm{V}+\mathrm{S}$ & SprP, NR & 1 & 4 & 38 \\
\hline & 6.4 & BAtr & Liquid drops & $1.0 \mathrm{E}+5$ & Macrofoam & DW & NR & Glass & 10 & PBSTr & $\mathrm{V}+\mathrm{S}$ & SprP, NR & 1 & 4 & 39 \\
\hline & 6.5 & BAtr & Liquid drops & $1.0 \mathrm{E}+5$ & Cotton & DW & NR & SS & 10 & PBSTr & $\mathrm{V}+\mathrm{S}$ & SprP, NR & 1 & 3 & 28 \\
\hline & 6.6 & BAtr & Liquid drops & $1.0 \mathrm{E}+5$ & Dacron & DW & NR & SS & 10 & PBSTr & $\mathrm{V}+\mathrm{S}$ & SprP, NR & 1 & 3 & 30 \\
\hline & 6.7 & BAtr & Liquid drops & $1.0 \mathrm{E}+5$ & Rayon & DW & NR & SS & 10 & PBSTr & $\mathrm{V}+\mathrm{S}$ & SprP, NR & 1 & 3 & 30 \\
\hline & 6.8 & BAtr & Liquid drops & $1.0 \mathrm{E}+5$ & Macrofoam & DW & NR & SS & 10 & PBSTr & $\mathrm{V}+\mathrm{S}$ & SprP, NR & 1 & 3 & 29 \\
\hline & 6.9 & BAtr & Liquid drops & $1.0 \mathrm{E}+5$ & Cotton & DW & NR & $\mathrm{PC}$ & 10 & PBSTr & $\mathrm{V}+\mathrm{S}$ & SprP, NR & 1 & 3 & 30 \\
\hline & 6.10 & Batr & Liquid drops & $1.0 \mathrm{E}+5$ & Dacron & DW & NR & PC & 10 & PBSTr & $\mathrm{V}+\mathrm{S}$ & SprP, NR & 1 & 3 & 30 \\
\hline & 6.11 & Batr & Liquid drops & $1.0 \mathrm{E}+5$ & Rayon & DW & NR & PC & 10 & PBSTr & $\mathrm{V}+\mathrm{S}$ & SprP, NR & 1 & 3 & 30 \\
\hline & 6.12 & BAtr & Liquid drops & $1.0 \mathrm{E}+5$ & Macrofoam & DW & NR & PC & 10 & PBSTr & $\mathrm{V}+\mathrm{S}$ & SprP, NR & 1 & 3 & 30 \\
\hline & 6.13 & BAtr & Liquid drops & $1.0 \mathrm{E}+5$ & Cotton & DW & NR & Vinyl & 10 & PBSTr & $\mathrm{V}+\mathrm{S}$ & SprP, NR & 1 & 3 & 29 \\
\hline & 6.14 & BAtr & Liquid drops & $1.0 \mathrm{E}+5$ & Dacron & DW & NR & Vinyl & 10 & PBSTr & $\mathrm{V}+\mathrm{S}$ & SprP, NR & 1 & 3 & 30 \\
\hline & 6.15 & BAtr & Liquid drops & $1.0 \mathrm{E}+5$ & Rayon & DW & NR & Vinyl & 10 & PBSTr & $\mathrm{V}+\mathrm{S}$ & SprP, NR & 1 & 3 & 29 \\
\hline & 6.16 & BAtr & Liquid drops & $1.0 \mathrm{E}+5$ & Macrofoam & DW & NR & Vinyl & 10 & PBSTr & $\mathrm{V}+\mathrm{S}$ & SprP, NR & 1 & 3 & 29 \\
\hline & 6.17 & BAtr & Dry aerosol & $1.0 \mathrm{E}+9$ & Cotton & DW & NR & Glass & 10 & PBSTr & $\mathrm{V}+\mathrm{S}$ & SprP, NR & 1 & 3 & 30 \\
\hline & 6.18 & BAtr & Dry aerosol & $1.0 \mathrm{E}+9$ & Dacron & DW & NR & Glass & 10 & PBSTr & $\mathrm{V}+\mathrm{S}$ & SprP, NR & 1 & 3 & 30 \\
\hline & 6.19 & BAtr & Dry aerosol & $1.0 \mathrm{E}+9$ & Rayon & DW & NR & Glass & 10 & PBSTr & $\mathrm{V}+\mathrm{S}$ & SprP, NR & 1 & 3 & 29 \\
\hline & 6.20 & BAtr & Dry aerosol & $1.0 \mathrm{E}+9$ & Macrofoam & DW & NR & Glass & 10 & PBSTr & $\mathrm{V}+\mathrm{S}$ & SprP, NR & 1 & 3 & 29 \\
\hline
\end{tabular}


Table A.1a Expanded summary of test conditions for swab sampling. Acronyms and abbreviations are defined in Table 6. (cont.)

\begin{tabular}{|c|c|c|c|c|c|c|c|c|c|c|c|c|c|c|c|}
\hline Reference & $\begin{array}{c}\text { Test } \\
\#\end{array}$ & Agent & $\begin{array}{c}\text { Agent } \\
\text { deposition }\end{array}$ & $\begin{array}{c}\text { Agent } \\
\text { concentration } \\
\left(\mathrm{CFU} / \mathrm{cm}^{2}\right)\end{array}$ & $\begin{array}{l}\text { Swab } \\
\text { type }\end{array}$ & $\begin{array}{c}\text { Wetting } \\
\text { agent }\end{array}$ & $\begin{array}{c}\text { Relative } \\
\text { humidity } \\
(\%)\end{array}$ & $\begin{array}{c}\text { Surface } \\
\text { type }\end{array}$ & $\begin{array}{l}\text { Surface } \\
\text { area } \\
\text { sampled } \\
\left(\mathrm{cm}^{2}\right) \\
\end{array}$ & $\begin{array}{c}\text { Extraction } \\
\text { liquid }\end{array}$ & $\begin{array}{c}\text { Extraction } \\
\text { method }\end{array}$ & $\begin{array}{l}\text { Culture } \\
\text { method, } \\
\text { medium }\end{array}$ & $\begin{array}{c}\# \\
\text { labs }\end{array}$ & $\begin{array}{c}\# \text { test } \\
\text { runs }\end{array}$ & $\begin{array}{c}\text { Total } \\
\text { \# test } \\
\text { samples }\end{array}$ \\
\hline \multirow{16}{*}{$\begin{array}{l}\text { Edmonds } \\
\text { et al. } 2009 \\
\text { (cont.) }\end{array}$} & 6.21 & BAtr & Dry aerosol & $1.0 \mathrm{E}+9$ & Cotton & DW & NR & SS & 10 & PBSTr & $\mathrm{V}+\mathrm{S}$ & SprP, NR & 1 & 3 & 30 \\
\hline & 6.22 & BAtr & Dry aerosol & $1.0 \mathrm{E}+9$ & Dacron & DW & NR & $\mathrm{SS}$ & 10 & PBSTr & $\mathrm{V}+\mathrm{S}$ & SprP, NR & 1 & 3 & 30 \\
\hline & 6.23 & BAtr & Dry aerosol & $1.0 \mathrm{E}+9$ & Rayon & DW & NR & SS & 10 & PBSTr & $\mathrm{V}+\mathrm{S}$ & SprP, NR & 1 & 3 & 30 \\
\hline & 6.24 & BAtr & Dry aerosol & $1.0 \mathrm{E}+9$ & Macrofoam & DW & NR & SS & 10 & PBSTr & $\mathrm{V}+\mathrm{S}$ & SprP, NR & 1 & 3 & 30 \\
\hline & 6.25 & BAtr & Dry aerosol & $1.0 \mathrm{E}+9$ & Cotton & DW & NR & $\mathrm{PC}$ & 10 & PBSTr & $\mathrm{V}+\mathrm{S}$ & SprP, NR & 1 & 3 & 30 \\
\hline & 6.26 & BAtr & Dry aerosol & $1.0 \mathrm{E}+9$ & Dacron & DW & NR & $\mathrm{PC}$ & 10 & PBSTr & $\mathrm{V}+\mathrm{S}$ & SprP, NR & 1 & 3 & 30 \\
\hline & 6.27 & BAtr & Dry aerosol & $1.0 \mathrm{E}+9$ & Rayon & DW & NR & $\mathrm{PC}$ & 10 & PBSTr & $\mathrm{V}+\mathrm{S}$ & SprP, NR & 1 & 3 & 30 \\
\hline & 6.28 & BAtr & Dry aerosol & $1.0 \mathrm{E}+9$ & Macrofoam & DW & NR & PC & 10 & PBSTr & $\mathrm{V}+\mathrm{S}$ & SprP, NR & 1 & 3 & 30 \\
\hline & 6.29 & BAtr & Dry aerosol & $1.0 \mathrm{E}+9$ & Cotton & DW & NR & Vinyl & 10 & PBSTr & $\mathrm{V}+\mathrm{S}$ & SprP, NR & 1 & 3 & 24 \\
\hline & 6.30 & BAtr & Dry aerosol & $1.0 \mathrm{E}+9$ & Dacron & DW & NR & Vinyl & 10 & PBSTr & $\mathrm{V}+\mathrm{S}$ & SprP, NR & 1 & 3 & 30 \\
\hline & 6.31 & BAtr & Dry aerosol & $1.0 \mathrm{E}+9$ & Rayon & DW & NR & Vinyl & 10 & PBSTr & $\mathrm{V}+\mathrm{S}$ & SprP, NR & 1 & 3 & 29 \\
\hline & 6.32 & BAtr & Dry aerosol & $1.0 \mathrm{E}+9$ & Macrofoam & DW & NR & Vinyl & 10 & PBSTr & $\mathrm{V}+\mathrm{S}$ & SprP, NR & 1 & 3 & 30 \\
\hline & 6.33 & BAtr & Liquid drops & 4774.35 & Dacron & DW & NR & Glass & 10 & PBSTr & $\mathrm{V}+\mathrm{S}$ & SprP, NR & 1 & 3 & 30 \\
\hline & 6.34 & BAtr & Liquid drops & 30114.6 & Dacron & DW & NR & Glass & 10 & PBSTr & $\mathrm{V}+\mathrm{S}$ & SprP, NR & 1 & 3 & 30 \\
\hline & 6.35 & BAtr & Liquid drops & $2.916 \mathrm{E}+5$ & Dacron & DW & NR & Glass & 10 & PBSTr & $\mathrm{V}+\mathrm{S}$ & SprP, NR & 1 & 3 & 30 \\
\hline & 6.36 & BAtr & Liquid drops & $2.524 \mathrm{E}+6$ & Dacron & DW & NR & Glass & 10 & PBSTr & $\mathrm{V}+\mathrm{S}$ & SprP, NR & 1 & 3 & 30 \\
\hline \multirow{20}{*}{$\begin{array}{l}\text { Valentine } \\
\text { et al. } 2008\end{array}$} & 7.1 & BS & Liquid drops & 90349.9 & Cotton & PBST & NR & Plastic & 104.04 & PBST & $\mathrm{V}$ & Plate, TSA & 1 & 10 & 10 \\
\hline & 7.2 & BS & Liquid drops & 90349.9 & PU foam & PBST & NR & Plastic & 104.04 & PBST & $\mathrm{V}$ & Plate, TSA & 1 & 10 & 10 \\
\hline & 7.3 & $\mathrm{BS}$ & Liquid drops & 90349.9 & Polyester & PBST & NR & Plastic & 104.04 & PBST & V & Plate, TSA & 1 & 10 & 10 \\
\hline & 7.4 & BS & Liquid drops & 90349.9 & Dacron & PBST & NR & Plastic & 104.04 & PBST & V & Plate, TSA & 1 & 10 & 10 \\
\hline & 7.5 & BS & Liquid drops & 90349.9 & Cotton & PBST & NR & Oak & 104.04 & PBST & V & Plate, TSA & 1 & 10 & 10 \\
\hline & 7.6 & $\mathrm{BS}$ & Liquid drops & 90349.9 & PU foam & PBST & NR & Oak & 104.04 & PBST & V & Plate, TSA & 1 & 10 & 10 \\
\hline & 7.7 & BS & Liquid drops & 90349.9 & Polyester & PBST & NR & Oak & 104.04 & PBST & V & Plate, TSA & 1 & 10 & 10 \\
\hline & 7.8 & BS & Liquid drops & 90349.9 & Dacron & PBST & NR & Oak & 104.04 & PBST & V & Plate, TSA & 1 & 10 & 10 \\
\hline & 7.9 & BS & Liquid drops & 282000 & Cotton & PBST & NR & Monitor & 25 & PBST & V & Plate, TSA & 1 & 10 & 10 \\
\hline & 7.10 & BS & Liquid drops & 282000 & PU foam & PBST & NR & Monitor & 25 & PBST & $\mathrm{V}$ & Plate, TSA & 1 & 10 & 10 \\
\hline & 7.11 & BS & Liquid drops & 282000 & Polyester & PBST & NR & Monitor & 25 & PBST & $\mathrm{V}$ & Plate, TSA & 1 & 10 & 10 \\
\hline & 7.12 & $\mathrm{BS}$ & Liquid drops & 282000 & Dacron & PBST & NR & Monitor & 25 & PBST & V & Plate, TSA & 1 & 10 & 10 \\
\hline & 7.13 & $\mathrm{BS}$ & Liquid drops & 90349.9 & Cotton & PBST & NR & PEUF & 104.04 & PBST & V & Plate, TSA & 1 & 10 & 10 \\
\hline & 7.14 & $\mathrm{BS}$ & Liquid drops & 90349.9 & PU foam & PBST & NR & PEUF & 104.04 & PBST & V & Plate, TSA & 1 & 10 & 10 \\
\hline & 7.15 & BS & Liquid drops & 90349.9 & Polyester & PBST & NR & PEUF & 104.04 & PBST & V & Plate, TSA & 1 & 10 & 10 \\
\hline & 7.16 & BS & Liquid drops & 90349.9 & Dacron & PBST & NR & PEUF & 104.04 & PBST & $\mathrm{V}$ & Plate, TSA & 1 & 10 & 10 \\
\hline & 7.17 & $\mathrm{BS}$ & Liquid drops & 90349.9 & Cotton & PBST & NR & Carpet & 104.04 & PBST & V & Plate, TSA & 1 & 10 & 10 \\
\hline & 7.18 & $\mathrm{BS}$ & Liquid drops & 90349.9 & PU foam & PBST & NR & Carpet & 104.04 & PBST & V & Plate, TSA & 1 & 10 & 10 \\
\hline & 7.19 & BS & Liquid drops & 90349.9 & Polyester & PBST & NR & Carpet & 104.04 & PBST & V & Plate, TSA & 1 & 10 & 10 \\
\hline & 7.20 & $\mathrm{BS}$ & Liquid drops & 90349.9 & Dacron & PBST & NR & Carpet & 104.04 & PBST & V & Plate, TSA & 1 & 10 & 10 \\
\hline \multirow{4}{*}{$\begin{array}{l}\text { Buttner et } \\
\text { al. } 2001\end{array}$} & 8.1 & $\mathrm{BS}$ & Liquid drops & $1.48 \mathrm{E}+6$ & Swab kit & PBST & NR & Glass & 5 & PBT & $\mathrm{V}$ & Plate, TSAC & 1 & 3 & 3 \\
\hline & 8.2 & BS & Liquid drops & $1.48 \mathrm{E}+6$ & Cotton & PBST & NR & Glass & 5 & PBT & $\mathrm{V}$ & Plate, TSAC & 1 & 3 & 3 \\
\hline & 8.3 & BS & Dry aerosol & $100-1000$ & Swab kit & PBST & NR & Vinyl & 32.49 & PBT & V & Plate, TSAC & 1 & 3 & 3 \\
\hline & 8.4 & $\mathrm{BS}$ & Dry aerosol & $100-1000$ & Swab kit & PBST & NR & Cpt-R & 32.49 & PBT & V & Plate, TSAC & 1 & 3 & 3 \\
\hline
\end{tabular}


Table A.1a Expanded summary of test conditions for swab sampling. Acronyms and abbreviations are defined in Table 6. (cont.)

\begin{tabular}{|c|c|c|c|c|c|c|c|c|c|c|c|c|c|c|c|}
\hline Reference & $\begin{array}{c}\text { Test } \\
\#\end{array}$ & Agent & $\begin{array}{c}\text { Agent } \\
\text { deposition }\end{array}$ & $\begin{array}{c}\text { Agent } \\
\text { concentration } \\
\left(\mathrm{CFU} / \mathrm{cm}^{2}\right)\end{array}$ & $\begin{array}{l}\text { Swab } \\
\text { type }\end{array}$ & $\begin{array}{c}\text { Wetting } \\
\text { agent }\end{array}$ & $\begin{array}{c}\text { Relative } \\
\text { humidity } \\
(\%)\end{array}$ & $\begin{array}{c}\text { Surface } \\
\text { type }\end{array}$ & $\begin{array}{l}\text { Surface } \\
\text { area } \\
\text { sampled } \\
\left(\mathrm{cm}^{2}\right) \\
\end{array}$ & $\begin{array}{c}\text { Extraction } \\
\text { liquid }\end{array}$ & $\begin{array}{c}\text { Extraction } \\
\text { method }\end{array}$ & $\begin{array}{l}\text { Culture } \\
\text { method, } \\
\text { medium }\end{array}$ & $\begin{array}{c}\# \\
\text { labs } \\
\end{array}$ & $\begin{array}{c}\# \text { test } \\
\text { runs }\end{array}$ & $\begin{array}{c}\text { Total } \\
\text { \# test } \\
\text { samples }\end{array}$ \\
\hline \multirow{14}{*}{$\begin{array}{l}\text { Buttner et } \\
\text { al. } 2001 \\
\text { (cont.) }\end{array}$} & 8.5 & $\mathrm{BS}$ & Dry aerosol & $100-1000$ & Swab kit & PBST & NR & Cpt-S & 32.49 & PBT & $\mathrm{V}$ & Plate, TSAC & 1 & 3 & 3 \\
\hline & 8.6 & $\mathrm{BS}$ & Dry aerosol & $100-1000$ & Swab kit & PBST & NR & Cpt-C & 32.49 & PBT & $\mathrm{V}$ & Plate, TSAC & 1 & 3 & 3 \\
\hline & 8.7 & BS & Dry aerosol & $100-1000$ & Cotton & PBST & NR & Vinyl & 32.49 & PBT & $\mathrm{V}$ & Plate, TSAC & 1 & 3 & 3 \\
\hline & 8.8 & BS & Dry aerosol & $100-1000$ & Cotton & PBST & NR & Cpt-R & 32.49 & PBT & $\mathrm{V}$ & Plate, TSAC & 1 & 3 & 3 \\
\hline & 8.9 & $\mathrm{BS}$ & Dry aerosol & $100-1000$ & Cotton & PBST & NR & Cpt-S & 32.49 & PBT & V & Plate, TSAC & 1 & 3 & 3 \\
\hline & 8.10 & BS & Dry aerosol & $100-1000$ & Cotton & PBST & NR & Cpt-C & 32.49 & PBT & V & Plate, TSAC & 1 & 3 & 3 \\
\hline & 8.11 & $\mathrm{BS}+\mathrm{PC} 2$ & Dry aerosol & $100-1000$ & Swab kit & PBST & NR & Vinyl & 32.49 & PBT & V & Plate, TSAC & 1 & 2 & 2 \\
\hline & 8.12 & $\mathrm{BS}+\mathrm{PC} 2$ & Dry aerosol & $100-1000$ & Swab kit & PBST & NR & Cpt-R & 32.49 & PBT & V & Plate, TSAC & 1 & 2 & 2 \\
\hline & 8.13 & $\mathrm{BS}+\mathrm{PC} 2$ & Dry aerosol & $100-1000$ & Swab kit & PBST & NR & Cpt-S & 32.49 & PBT & V & Plate, TSAC & 1 & 2 & 2 \\
\hline & 8.14 & $\mathrm{BS}+\mathrm{PC} 2$ & Dry aerosol & $100-1000$ & Swab kit & PBST & NR & Cpt-C & 32.49 & PBT & V & Plate, TSAC & 1 & 2 & 2 \\
\hline & 8.15 & $\mathrm{BS}+\mathrm{PC} 4$ & Dry aerosol & $100-1000$ & Swab kit & PBST & NR & Vinyl & 32.49 & PBT & $\mathrm{V}$ & Plate, TSAC & 1 & 2 & 2 \\
\hline & 8.16 & $\mathrm{BS}+\mathrm{PC} 4$ & Dry aerosol & $100-1000$ & Swab kit & PBST & NR & Cpt-R & 32.49 & PBT & $\mathrm{V}$ & Plate, TSAC & 1 & 2 & 2 \\
\hline & 8.17 & $\mathrm{BS}+\mathrm{PC} 4$ & Dry aerosol & $100-1000$ & Swab kit & PBST & NR & Cpt-S & 32.49 & PBT & V & Plate, TSAC & 1 & 2 & 2 \\
\hline & 8.18 & $\mathrm{BS}+\mathrm{PC} 4$ & Dry aerosol & $100-1000$ & Swab kit & PBST & NR & Cpt-C & 32.49 & PBT & $\mathrm{V}$ & Plate, TSAC & 1 & 2 & 2 \\
\hline \multirow{9}{*}{$\begin{array}{l}\text { Buttner et } \\
\text { al. 2004a }\end{array}$} & 9.1 & BAtr & Dry aerosol & $107.6-1076.4$ & SSPK & NR & NR & Vinyl & 929 & NR & HM & Plate, TSA & 1 & 3 & 3 \\
\hline & 9.2 & BAtr & Dry aerosol & $107.6-1076.4$ & SSPK & NR & NR & Wood & 929 & NR & $\mathrm{HM}$ & Plate, TSA & 1 & 3 & 3 \\
\hline & 9.3 & BAtr & Dry aerosol & $107.6-1076.4$ & SSPK & NR & NR & Metal & 929 & NR & $\mathrm{HM}$ & Plate, TSA & 1 & 3 & 3 \\
\hline & 9.4 & BAtr & Dry aerosol & $107.6-1076.4$ & SSPK & NR & NR & Vinyl & 929 & NR & HM & Plate, TSA & 1 & 3 & 3 \\
\hline & 9.5 & BAtr & Dry aerosol & $107.6-1076.4$ & SSPK & NR & NR & Wood & 929 & NR & HM & Plate, TSA & 1 & 3 & 3 \\
\hline & 9.6 & BAtr & Dry aerosol & $107.6-1076.4$ & SSPK & NR & NR & Metal & 929 & NR & $\mathrm{HM}$ & Plate, TSA & 1 & 3 & 3 \\
\hline & 9.7 & BAtr & Dry aerosol & $107.6-1076.4$ & SSPK & NR & NR & Vinyl & 929 & NR & $\mathrm{HM}$ & Plate, TSA & 1 & 1 & 2 \\
\hline & 9.8 & BAtr & Dry aerosol & $107.6-1076.4$ & SSPK & NR & NR & Wood & 929 & NR & $\mathrm{HM}$ & Plate, TSA & 1 & 1 & 2 \\
\hline & 9.9 & BAtr & Dry aerosol & $107.6-1076.4$ & SSPK & NR & NR & Metal & 929 & NR & $\mathrm{HM}$ & Plate, TSA & 1 & 1 & 2 \\
\hline \multirow{4}{*}{$\begin{array}{l}\text { Buttner et } \\
\text { al. } 2004 \mathrm{~b}\end{array}$} & 10.1 & BAtr & Dry aerosol & NR & SSPK & NR & NR & Metal & 317 & NR & HM & Plate, TSA & 1 & NR & 4 \\
\hline & 10.2 & BAtr & Dry aerosol & NR & SSPK & NR & NR & Wood & 317 & NR & HM & Plate, TSA & 1 & NR & 8 \\
\hline & 10.3 & BAtr & Dry aerosol & NR & Cotton & NR & NR & Metal & 100 & NR & Shake & Plate, TSA & 1 & NR & 4 \\
\hline & 10.4 & BAtr & Dry aerosol & NR & Cotton & NR & NR & Wood & 100 & NR & Shake & Plate, TSA & 1 & NR & 8 \\
\hline \multirow{4}{*}{$\begin{array}{l}\text { Quizon et } \\
\text { al. } 2007\end{array}$} & 11.1 & BAtr & Wet aerosol & NR & PU foam & PBST & NR & PWB & 100 & PBST & $\mathrm{V}+\mathrm{S}$ & SP/FP, TSA & 1 & 4 & 4 \\
\hline & 11.2 & BAtr & Wet aerosol & NR & PU foam & PBST & NR & SS & 100 & PBST & $\mathrm{V}+\mathrm{S}$ & SP/FP, TSA & 1 & 4 & 10 \\
\hline & 11.3 & BAtr & Wet aerosol & NR & PU foam & PBST & NR & Vinyl & 100 & PBST & $\mathrm{V}+\mathrm{S}$ & SP/FP, TSA & 1 & 4 & 4 \\
\hline & 11.4 & BAtr & Wet aerosol & NR & PU foam & PBST & NR & Wood & 100 & PBST & $\mathrm{V}+\mathrm{S}$ & SP/FP, TSA & 1 & 4 & 4 \\
\hline \multirow{9}{*}{$\begin{array}{l}\text { Nellen et } \\
\text { al. } 2006\end{array}$} & 12.1 & BS 168 & Liquid drops & $1000 \mathrm{CFU} / \mathrm{swab}$ & Cotton, DI & NA & NR & NA & NA & PBS & Untreated & Plate, TSA & 1 & 3 & 15 \\
\hline & 12.2 & BS 168 & Liquid drops & $1000 \mathrm{CFU} / \mathrm{swab}$ & Rayon, DI & NA & NR & NA & NA & PBS & Untreated & Plate, TSA & 1 & 3 & 15 \\
\hline & 12.3 & BS 168 & Liquid drops & $1000 \mathrm{CFU} / \mathrm{swab}$ & Cotton, DI & NA & NR & NA & NA & PBS & $\mathrm{V}$ & Plate, TSA & 1 & 3 & 15 \\
\hline & 12.4 & BS 168 & Liquid drops & $1000 \mathrm{CFU} / \mathrm{swab}$ & Rayon, DI & NA & NR & NA & NA & PBS & V & Plate, TSA & 1 & 3 & 15 \\
\hline & 12.5 & BS 168 & Liquid drops & $1000 \mathrm{CFU} / \mathrm{swab}$ & Cotton, DI & NA & NR & NA & NA & PBS & $\mathrm{S}$ & Plate, TSA & 1 & 3 & 15 \\
\hline & 12.6 & BS 168 & Liquid drops & $1000 \mathrm{CFU} / \mathrm{swab}$ & Rayon, DI & NA & NR & NA & NA & PBS & $\mathrm{S}$ & Plate, TSA & 1 & 3 & 15 \\
\hline & 12.7 & BS 168 & Liquid drops & $1000 \mathrm{CFU} / \mathrm{swab}$ & Cotton, DI & NA & NR & NA & NA & PBS & $\mathrm{V}+\mathrm{S}$ & Plate, TSA & 1 & 3 & 15 \\
\hline & 12.8 & BS 168 & Liquid drops & $1000 \mathrm{CFU} / \mathrm{swab}$ & Rayon, DI & NA & NR & NA & NA & PBS & $\mathrm{V}+\mathrm{S}$ & Plate, TSA & 1 & 3 & 15 \\
\hline & 12.9 & BS 168 & Liquid drops & 4 & Cotton & DW & NR & Petri dish & 25 & PBS & NR (c) & Plate, TSA & 1 & 3 & 15 \\
\hline
\end{tabular}


Table A.1a Expanded summary of test conditions for swab sampling. Acronyms and abbreviations are defined in Table 6. (cont.)

\begin{tabular}{|c|c|c|c|c|c|c|c|c|c|c|c|c|c|c|c|}
\hline Reference & $\begin{array}{c}\text { Test } \\
\# \\
\end{array}$ & Agent & $\begin{array}{c}\text { Agent } \\
\text { deposition }\end{array}$ & $\begin{array}{c}\text { Agent } \\
\text { concentration } \\
\left(\mathrm{CFU} / \mathrm{cm}^{2}\right) \\
\end{array}$ & $\begin{array}{l}\text { Swab } \\
\text { type }\end{array}$ & $\begin{array}{c}\text { Wetting } \\
\text { agent }\end{array}$ & $\begin{array}{c}\text { Relative } \\
\text { humidity } \\
(\%)\end{array}$ & $\begin{array}{c}\text { Surface } \\
\text { type }\end{array}$ & $\begin{array}{c}\text { Surface } \\
\text { area } \\
\text { sampled } \\
\left(\mathrm{cm}^{2}\right) \\
\end{array}$ & $\begin{array}{c}\text { Extraction } \\
\text { liquid }\end{array}$ & $\begin{array}{c}\text { Extraction } \\
\text { method }\end{array}$ & $\begin{array}{l}\text { Culture } \\
\text { method, } \\
\text { medium }\end{array}$ & $\begin{array}{c}\# \\
\text { labs }\end{array}$ & $\begin{array}{l}\# \text { test } \\
\text { runs }\end{array}$ & $\begin{array}{r}\text { Total } \\
\text { \# test } \\
\text { samples }\end{array}$ \\
\hline \multirow{13}{*}{$\begin{array}{l}\text { Nellen et } \\
\text { al. } 2006 \\
\text { (cont.) }\end{array}$} & 12.10 & BS 168 & Liquid drops & 4 & Rayon & DW & $\mathrm{NR}$ & Petri dish & 25 & PBS & NR (c) & Plate, TSA & 1 & 3 & 15 \\
\hline & 12.11 & BS 168 & Liquid drops & 4 & Cotton & DW & NR & Petri dish & 25 & PBS & $\mathrm{H}+\mathrm{V}+\mathrm{S}$ & Plate, TSA & 1 & 3 & 15 \\
\hline & 12.12 & BS 168 & Liquid drops & 4 & Rayon & DW & NR & Petri dish & 25 & PBS & $\mathrm{H}+\mathrm{V}+\mathrm{S}$ & Plate, TSA & 1 & 3 & 15 \\
\hline & 12.13 & BS 168 & Liquid drops & 16 & Rayon & DW & NR & Alum & 25 & PBS & NR (c) & Plate, TSA & 1 & 3 & 3 \\
\hline & 12.14 & BS 168 & Liquid drops & 16 & Rayon & DW & NR & V2A steel & 25 & PBS & NR (c) & Plate, TSA & 1 & 3 & 3 \\
\hline & 12.15 & BS 168 & Liquid drops & 16 & Rayon & DW & NR & MLI foil & 25 & PBS & NR (c) & Plate, TSA & 1 & 3 & 3 \\
\hline & 12.16 & BS 168 & Liquid drops & 16 & Rayon & DW & NR & Kapton & 25 & PBS & NR (c) & Plate, TSA & 1 & 3 & 3 \\
\hline & 12.17 & BS 168 & Liquid drops & 16 & Rayon & DW & NR & Teflon & 25 & PBS & NR (c) & Plate, TSA & 1 & 3 & 3 \\
\hline & 12.18 & BS 168 & Liquid drops & 16 & Rayon & DW & NR & Alum & 25 & PBS & $\mathrm{H}+\mathrm{V}+\mathrm{S}$ & Plate, TSA & 1 & 3 & 3 \\
\hline & 12.19 & BS 168 & Liquid drops & 16 & Rayon & DW & NR & V2A steel & 25 & PBS & $\mathrm{H}+\mathrm{V}+\mathrm{S}$ & Plate, TSA & 1 & 3 & 3 \\
\hline & 12.20 & BS 168 & Liquid drops & 16 & Rayon & DW & NR & MLI foil & 25 & PBS & $\mathrm{H}+\mathrm{V}+\mathrm{S}$ & Plate, TSA & 1 & 3 & 3 \\
\hline & 12.21 & BS 168 & Liquid drops & 16 & Rayon & DW & NR & Kapton & 25 & PBS & $\mathrm{H}+\mathrm{V}+\mathrm{S}$ & Plate, TSA & 1 & 3 & 3 \\
\hline & 12.22 & BS 168 & Liquid drops & 16 & Rayon & DW & NR & Teflon & 25 & PBS & $\mathrm{H}+\mathrm{V}+\mathrm{S}$ & Plate, TSA & 1 & 3 & 3 \\
\hline \multirow{12}{*}{$\begin{array}{l}\text { Hodges et } \\
\text { al. } 2010\end{array}$} & 13.1 & BA Sterne & Liquid drops & 1.88 & Macrofoam & PBST & NR & SS & 26 & PBST & $\mathrm{V}$ & FP, TSAB & 12 & 1 & 118 \\
\hline & 13.2 & BA Sterne & Liquid drops & 19.46 & Macrofoam & PBST & NR & SS & 26 & PBST & V & FP, TSAB & 12 & 1 & 120 \\
\hline & 13.3 & BA Sterne & Liquid drops & 1607.2 & Macrofoam & PBST & NR & SS & 26 & PBST & $\mathrm{V}$ & FP, TSAB & 12 & 1 & 116 \\
\hline & 13.4 & BA Sterne & Liquid imm. & $42 \mathrm{CFU} / \mathrm{swab}$ & Mfoam, DI & NA & NR & NA & NA & PBST & $\mathrm{V}$ & FP, TSAB & $10 / 12$ & 1 & $30 / 24^{(\mathrm{f})}$ \\
\hline & 13.5 & BA Sterne & Liquid imm. & $373 \mathrm{CFU} / \mathrm{swab}$ & Mfoam, DI & NA & NR & NA & NA & PBST & $\mathrm{V}$ & FP, TSAB & $10 / 12$ & 1 & $30 / 24^{(f)}$ \\
\hline & 13.6 & BA Sterne & Liquid imm. & $33300 \mathrm{CFU} / \mathrm{swab}$ & Mfoam, DI & NA & NR & NA & NA & PBST & $\mathrm{V}$ & $\mathrm{FP}, \mathrm{TSAB}$ & $10 / 10$ & 1 & $30 / 20^{(f)}$ \\
\hline & 13.7 & BA Sterne & Liquid drops & 1.38 & Macrofoam & (d) & NR & SS & 26 & PBST & $\mathrm{V}$ & FP, TSAB & 12 & 1 & 120 \\
\hline & 13.8 & BA Sterne & Liquid drops & 15.27 & Macrofoam & (d) & NR & SS & 26 & PBST & $\mathrm{V}$ & FP, TSAB & 12 & 1 & 120 \\
\hline & 13.9 & BA Sterne & Liquid drops & 1188.5 & Macrofoam & (d) & NR & SS & 26 & PBST & $\mathrm{V}$ & FP, TSAB & 12 & 1 & 120 \\
\hline & 13.10 & BA Sterne & Liquid imm. & $36 \mathrm{CFU} / \mathrm{swab}$ & Mfoam, DI & (d) & NR & NA & 26 & PBST & $\mathrm{V}$ & FP, TSAB & 12 & 1 & $24 / 48^{(\mathrm{e})}$ \\
\hline & 13.11 & BA Sterne & Liquid imm. & $397 \mathrm{CFU} / \mathrm{swab}$ & Mfoam, DI & (d) & NR & NA & 26 & PBST & $\mathrm{V}$ & FP, TSAB & 12 & 1 & $24 / 48^{(\mathrm{e})}$ \\
\hline & 13.12 & BA Sterne & Liquid imm. & $30900 \mathrm{CFU} / \mathrm{swab}$ & Mfoam, DI & (d) & NR & NA & 26 & PBST & $\mathrm{V}$ & FP, TSAB & 12 & 1 & $24 / 48^{(\mathrm{e})}$ \\
\hline
\end{tabular}

(a) Five analysts from two laboratories each analyzed samples with each contaminant concentration.

(b) $1 \mathrm{E}+2-1 \mathrm{E}+5 /$ sample, sample surface area not reported.

(c) These tests investigated the spores detached from the surface by swabbing, although the method used to quantify spore detachment independent of extraction and processing

was not described by Nellen et al. (2006).

(d) PBST + Arizona Test Dust + Bacillus atrophaeus + Staphylococcus epidermidis.

(e) $\mathrm{xx} / \mathrm{yy}=\#$ positive control samples/\# negative control samples. 
Table A.1b Expanded summary of test results for swab sampling. Acronyms and abbreviations are defined in Table 6 .

\begin{tabular}{|c|c|c|c|c|c|c|c|c|c|c|c|c|c|c|c|c|c|}
\hline \multirow[b]{2}{*}{ Reference } & \multirow[b]{2}{*}{$\begin{array}{c}\text { Test } \\
\#\end{array}$} & \multicolumn{5}{|c|}{$\begin{array}{c}\text { Recovery concentration results - } \\
\text { Mean \& \%RSDs }\end{array}$} & \multicolumn{5}{|c|}{$\begin{array}{l}\text { Recovery efficiency (RE) - } \\
\text { Mean \& \%RSDs } \\
\end{array}$} & \multicolumn{6}{|c|}{ LOD, FNR, and FPR } \\
\hline & & $\begin{array}{c}\text { Mean } \\
\left(\mathrm{CFU} / \mathrm{cm}^{2}\right)\end{array}$ & $\begin{array}{l}\text { Lab } \\
\% \text { RSD }\end{array}$ & $\begin{array}{l}\text { Run } \\
\% \text { RSD }\end{array}$ & $\begin{array}{c}\text { Sample- } \\
\text { within-run } \\
\% \text { RSD } \\
\end{array}$ & $\begin{array}{c}\text { Total } \\
\% \text { RSD }\end{array}$ & $\begin{array}{c}\mathrm{RE} \\
\text { mean } \\
(\%) \\
\end{array}$ & $\begin{array}{l}\text { RE lab } \\
\text { \%RSD } \\
\end{array}$ & $\begin{array}{l}\text { RE run } \\
\% \text { RSD }\end{array}$ & $\begin{array}{c}\text { RE sample- } \\
\text { within-run } \\
\% \text { RSD } \\
\end{array}$ & \begin{tabular}{|c|} 
RE \\
total \\
$\% R S D$
\end{tabular} & $\begin{array}{c}\text { Positive } \\
\text { Result } \\
(\mathrm{CFU}) \\
\end{array}$ & $\begin{array}{c}\text { LOD } \\
\text { defin- } \\
\text { ition } \\
\end{array}$ & $\begin{array}{c}\text { LOD } \\
\left(\mathrm{CFU} / \mathrm{cm}^{2}\right) \\
\end{array}$ & $\begin{array}{c}\text { LOD SD } \\
\text { or } 95 \% \text { CI } \\
\left(\mathrm{CFU} / \mathrm{cm}^{2}\right) \\
\end{array}$ & FNR & FPR \\
\hline Estill et & 1.1 & 0.0012 & 110 & 0 & 460 & 473 & 3.4 & 68 & 0 & 550 & 560 & & & & & & \\
\hline \multirow[t]{5}{*}{ al. 2009} & 1.2 & 0.025 & 0 & 27 & 82 & 86 & 6.5 & 0 & 12 & 80 & 81 & $\geq 1$ & $\mathrm{LOD}_{95}$ & 1.9 & $\begin{array}{l}95 \% \text { CI: } \\
074-37\end{array}$ & NR & $\begin{array}{l}1 / 27= \\
0.037\end{array}$ \\
\hline & 1.3 & 0.15 & 19 & 87 & 75 & 116 & 5.1 & 21 & 67 & 78 & 100 & & & & & & \\
\hline & 1.4 & 0.0018 & 85 & 0 & 220 & 236 & 12.0 & 110 & 0 & 230 & 260 & & & & & & \\
\hline & 1.5 & 0.023 & 0 & 98 & 73 & 122 & 14.0 & 0 & 110 & 76 & 130 & $\geq 1$ & $\mathrm{LOD}_{95}$ & 0.4 & $95 \%$ Cl: & NR & $1 / 27=$ \\
\hline & 1.6 & 0.18 & 21 & 40 & 57 & 73 & 12.0 & 19 & 71 & 76 & 110 & & & & & & \\
\hline \multirow{6}{*}{$\begin{array}{l}\text { Hodges et } \\
\text { al. } 2006\end{array}$} & 2.1 & 0.1 & NR & NR & 100 & NR & 31.7 & NA & NA & 82 & NA & $\geq 1$ & & & & 0.267 & NR \\
\hline & 2.2 & 0.2 & NR & NR & 100 & NR & 37.8 & NA & NA & 91 & NA & $\geq 1$ & & & & 0.133 & NR \\
\hline & 2.3 & 0.4 & NR & NR & 75 & NR & 37.2 & NA & NA & 58 & NA & $\geq 1$ & $\mathrm{LOD}_{90}$ & 1.2 & NR & 0 & NR \\
\hline & 2.4 & 1.5 & NR & NR & 27 & NR & 40.1 & NA & 32 & 23 & NA & $\geq 1$ & & & & 0 & NR \\
\hline & 2.5 & 22 & NR & NR & 30 & NR & 38.0 & NA & 28 & 21 & NA & $\geq 1$ & & & & 0 & NR \\
\hline & 2.6 & 2900 & NR & NR & 30 & NR & 49.1 & NA & 33 & 27 & NA & $\geq 1$ & & & & 0 & NR \\
\hline \multirow{25}{*}{$\begin{array}{l}\text { Rose et } \\
\text { al. } 2004\end{array}$} & 3.1 & NR & NA & $\mathrm{NR}$ & NR & NA & 0.5 & NA & NR & 80 & NA & $\mathrm{NR}$ & NR & NR & NR & NR & NR \\
\hline & 3.2 & NR & NA & NR & NR & NA & 4.7 & NA & NR & 47 & NA & NR & NR & NR & NR & NR & NR \\
\hline & 3.3 & NR & NA & NR & NR & NA & 8.0 & NA & NR & 18 & NA & NR & NR & NR & NR & NR & NR \\
\hline & 3.4 & NR & NA & NR & NR & NA & 41.7 & NA & NR & 35 & NA & NR & NR & NR & NR & NR & NR \\
\hline & 3.5 & NR & NA & NR & NR & NA & 6.9 & NA & NR & 44 & NA & NR & NR & NR & NR & NR & NR \\
\hline & 3.6 & NR & NA & NR & NR & NA & 13.6 & NA & NR & 24 & NA & NR & NR & NR & NR & NR & NR \\
\hline & 3.7 & NR & NA & NR & NR & NA & 0.1 & NA & NR & 200 & NA & NR & NR & NR & NR & NR & NR \\
\hline & 3.8 & NR & NA & NR & NR & NA & 2.0 & NA & NR & 50 & NA & NR & NR & NR & NR & NR & NR \\
\hline & 3.9 & NR & NA & NR & NR & NA & 2.1 & NA & NR & 43 & NA & NR & NR & NR & NR & NR & NR \\
\hline & 3.10 & NR & NA & NR & NR & NA & 9.9 & NA & NR & 38 & NA & NR & NR & NR & NR & NR & NR \\
\hline & 3.11 & NR & NA & NR & NR & NA & 1.4 & NA & NR & 36 & NA & NR & NR & NR & NR & NR & NR \\
\hline & 3.12 & NR & NA & NR & NR & NA & 11.2 & NA & NR & 39 & NA & NR & NR & NR & NR & NR & NR \\
\hline & 3.13 & NR & NA & NR & NR & NA & 0.1 & NA & NR & 200 & NA & NR & NR & NR & NR & NR & NR \\
\hline & 3.14 & NR & NA & $\mathrm{NR}$ & NR & NA & 1.0 & NA & NR & 80 & NA & NR & NR & NR & NR & NR & NR \\
\hline & 3.15 & NR & NA & NR & NR & NA & 4.4 & NA & NR & 23 & NA & NR & NR & NR & NR & NR & NR \\
\hline & 3.16 & NR & NA & NR & NR & NA & 11.5 & NA & NR & 69 & NA & NR & NR & NR & NR & NR & NR \\
\hline & 3.17 & NR & NA & NR & NR & NA & 4.5 & NA & NR & 22 & NA & NR & NR & NR & NR & NR & NR \\
\hline & 3.18 & NR & NA & NR & NR & NA & 8.5 & NA & NR & 52 & NA & NR & NR & NR & NR & NR & NR \\
\hline & 3.19 & NR & NA & NR & NR & NA & 0.7 & NA & NR & 157 & NA & NR & NR & NR & NR & NR & NR \\
\hline & 3.20 & NR & NA & NR & NR & NA & 6.3 & NA & NR & 62 & NA & NR & NR & NR & NR & NR & NR \\
\hline & 3.21 & NR & NA & NR & NR & NA & 11.9 & NA & NR & 26 & NA & NR & NR & NR & NR & NR & NR \\
\hline & 3.22 & NR & NA & NR & NR & NA & 43.6 & NA & NR & 26 & NA & NR & NR & NR & NR & NR & NR \\
\hline & 3.23 & NR & NA & NR & NR & NA & 12.7 & NA & NR & 27 & NA & NR & NR & NR & NR & NR & NR \\
\hline & 3.24 & NR & NA & NR & NR & NA & 17.7 & NA & NR & 33 & NA & NR & NR & NR & NR & NR & NR \\
\hline & 3.25 & NR & NA & NR & NR & NA & 93.9 & NA & NR & 11 & NA & NR & NR & NR & NR & NR & NR \\
\hline
\end{tabular}


Table A.1b Expanded summary of test results for swab sampling. Acronyms and abbreviations are defined in Table 6. (cont.)

\begin{tabular}{|c|c|c|c|c|c|c|c|c|c|c|c|c|c|c|c|c|c|}
\hline \multirow[b]{2}{*}{ Reference } & \multirow[b]{2}{*}{$\begin{array}{c}\text { Test } \\
\#\end{array}$} & \multicolumn{5}{|c|}{$\begin{array}{c}\text { Recovery concentration results - } \\
\text { Mean \& \%RSDs }\end{array}$} & \multicolumn{5}{|c|}{$\begin{array}{c}\text { Recovery efficiency }(\mathrm{RE})- \\
\text { Mean \& \%RSDs }\end{array}$} & \multicolumn{6}{|c|}{ LOD, FNR, and FPR } \\
\hline & & $\begin{array}{c}\text { Mean } \\
\left(\mathrm{CFU} / \mathrm{cm}^{2}\right)\end{array}$ & $\begin{array}{l}\text { Lab } \\
\% \text { RSD }\end{array}$ & $\begin{array}{l}\text { Run } \\
\% \text { RSD }\end{array}$ & $\begin{array}{c}\text { Sample- } \\
\text { within-run } \\
\% \text { RSD }\end{array}$ & $\begin{array}{c}\text { Total } \\
\% \text { RSD }\end{array}$ & \begin{tabular}{|c}
$\mathrm{RE}$ \\
mean \\
$(\%)$ \\
\end{tabular} & $\begin{array}{l}\text { RE lab } \\
\text { \%RSD }\end{array}$ & $\begin{array}{l}\text { RE run } \\
\% \text { RSD }\end{array}$ & $\begin{array}{c}\text { RE sample- } \\
\text { within-run } \\
\% \text { RSD }\end{array}$ & \begin{tabular}{|c|}
$\mathrm{RE}$ \\
total \\
$\% \mathrm{RSD}$ \\
\end{tabular} & $\begin{array}{c}\text { Positive } \\
\text { result } \\
(\mathrm{CFU})\end{array}$ & $\begin{array}{l}\text { LOD } \\
\text { defin- } \\
\text { ition }\end{array}$ & $\begin{array}{c}\text { LOD } \\
\left(\mathrm{CFU} / \mathrm{cm}^{2}\right)\end{array}$ & $\begin{array}{c}\text { LOD SD } \\
\text { or } 95 \% \text { CI } \\
\left(\mathrm{CFU} / \mathrm{cm}^{2}\right)\end{array}$ & FNR & FPR \\
\hline \multirow{3}{*}{$\begin{array}{l}\text { Rose et. } \\
\text { al. } 2004 \\
\text { (cont.) }\end{array}$} & 3.26 & NR & NA & NR & NR & NA & 83.8 & NA & NR & 9 & NA & NR & NR & NR & NR & NR & NR \\
\hline & 3.27 & NR & NA & NR & NR & NA & 91.7 & NA & NR & 7 & NA & NR & NR & NR & NR & NR & NR \\
\hline & 3.28 & NR & NA & NR & NR & NA & 93.4 & NA & NR & 12 & NA & NR & NR & NR & NR & NR & NR \\
\hline \multirow{13}{*}{$\begin{array}{l}\text { Frawley et } \\
\text { al. } 2008\end{array}$} & 4.1 & NR & NA & NR & NR & NA & 2.3 & NA & NR & 30 & NA & NR & NR & NR & NR & NR & NR \\
\hline & 4.2 & NR & NA & NR & NR & NA & 0.2 & NA & NR & 56 & NA & NR & NR & NR & NR & NR & NR \\
\hline & 4.3 & NR & NA & NR & NR & NA & 0.6 & NA & NR & 71 & NA & NR & NR & NR & NR & NR & NR \\
\hline & 4.4 & NR & NA & NR & NR & NA & 5.5 & NA & NR & 42 & NA & NR & NR & NR & NR & NR & NR \\
\hline & 4.5 & NR & NA & NR & NR & NA & 2.5 & NA & NR & 63 & NA & NR & NR & NR & NR & NR & NR \\
\hline & 4.6 & NR & NA & NR & NR & NA & 2.0 & NA & NR & 61 & NA & NR & NR & NR & NR & NR & NR \\
\hline & 4.7 & NR & NA & NR & NR & NA & 8.0 & NA & NR & 45 & NA & NR & NR & NR & NR & NR & NR \\
\hline & 4.8 & NR & NA & NR & NR & NA & 15.0 & NA & NR & 39 & NA & NR & NR & NR & NR & NR & NR \\
\hline & 4.9 & NR & NA & NR & NR & NA & 15.0 & NA & NR & 37 & NA & NR & NR & NR & NR & NR & NR \\
\hline & 4.10 & NR & NA & NR & NR & NA & 14.0 & NA & NR & 28 & NA & NR & NR & NR & NR & NR & NR \\
\hline & 4.11 & NR & NA & NR & NR & NA & 2.0 & NA & NR & 95 & NA & NR & NR & NR & NR & NR & NR \\
\hline & 4.12 & NR & NA & NR & NR & NA & 2.0 & NA & NR & 144 & NA & NR & NR & NR & NR & NR & NR \\
\hline & 4.13 & NR & NA & NR & NR & NA & 0.0 & NA & NR & NA & NA & NR & NR & NR & NR & NR & NR \\
\hline \multirow{6}{*}{$\begin{array}{l}\text { Brown et } \\
\text { al. } 2007 b\end{array}$} & 5.1 & NR & NA & NR & NR & NA & 39.5 & NA & NR & 50 & NA & $\geq 1 \mathrm{CFU} / \mathrm{ml}$ & NR & 1 & NR & NR & NR \\
\hline & 5.2 & NR & NA & NR & NR & NA & 42.9 & NA & NR & 34 & NA & $\geq 1 \mathrm{CFU} / \mathrm{ml}$ & NR & 1 & NR & NR & NR \\
\hline & 5.3 & NR & NA & NR & NR & NA & 35.5 & NA & NR & 67 & NA & $\geq 1 \mathrm{CFU} / \mathrm{ml}$ & NR & 1 & NR & NR & NR \\
\hline & 5.4 & NR & NA & NR & NR & NA & 45.6 & NA & NR & 48 & NA & $\geq 1 \mathrm{CFU} / \mathrm{ml}$ & NR & 1 & NR & NR & NR \\
\hline & 5.5 & NR & NA & NR & NR & NA & 75.6 & NA & NR & 16 & NA & NR & NR & NR & NR & NR & NR \\
\hline & 5.6 & NR & NA & NR & NR & NA & 99.9 & NA & NR & 0.1 & NA & NR & NR & NR & NR & NR & NR \\
\hline \multirow{16}{*}{$\begin{array}{l}\text { Edmonds } \\
\text { et al. } 2009\end{array}$} & 6.1 & NR & NA & NR & NR & NA & 88.7 & NA & 11 & 9 & NA & NR & NR & NR & NR & NR & NR \\
\hline & 6.2 & NR & NA & NR & NR & NA & 82.1 & NA & 14 & 11 & NA & NR & NR & NR & NR & NR & NR \\
\hline & 6.3 & NR & NA & NR & NR & NA & 87.5 & NA & 14 & 13 & NA & NR & NR & NR & NR & NR & NR \\
\hline & 6.4 & NR & NA & NR & NR & NA & 89.1 & NA & 19 & 10 & NA & NR & NR & NR & NR & NR & NR \\
\hline & 6.5 & NR & NA & NR & NR & NA & 47.0 & NA & 20 & 15 & NA & NR & NR & NR & NR & NR & NR \\
\hline & 6.6 & NR & NA & NR & NR & NA & 42.5 & NA & 21 & 16 & NA & NR & NR & NR & NR & NR & NR \\
\hline & 6.7 & NR & NA & NR & NR & NA & 43.6 & NA & 33 & 16 & NA & NR & NR & NR & NR & NR & NR \\
\hline & 6.8 & NR & NA & NR & NR & NA & 55.7 & NA & 19 & 15 & NA & NR & NR & NR & NR & NR & NR \\
\hline & 6.9 & NR & NA & NR & NR & NA & 74.9 & NA & 16 & 10 & NA & NR & NR & NR & NR & NR & NR \\
\hline & 6.10 & NR & NA & NR & NR & NA & 83.4 & NA & 17 & 10 & NA & NR & NR & NR & NR & NR & NR \\
\hline & 6.11 & NR & NA & NR & NR & NA & 75.4 & NA & 16 & 11 & NA & NR & NR & NR & NR & NR & NR \\
\hline & 6.12 & NR & NA & NR & NR & NA & 88.3 & NA & 19 & 8 & NA & NR & NR & NR & NR & NR & NR \\
\hline & 6.13 & NR & NA & NR & NR & NA & 49.0 & NA & 24 & 13 & NA & NR & NR & NR & NR & NR & NR \\
\hline & 6.14 & NR & NA & NR & NR & NA & 62.2 & NA & 34 & 13 & NA & NR & NR & NR & NR & NR & NR \\
\hline & 6.15 & NR & NA & NR & NR & NA & 58.3 & NA & 29 & 11 & NA & NR & NR & NR & NR & NR & NR \\
\hline & 6.16 & NR & NA & NR & NR & NA & 72.0 & NA & 26 & 11 & NA & NR & NR & NR & NR & NR & NR \\
\hline
\end{tabular}


Table A.1b Expanded summary of test results for swab sampling. Acronyms and abbreviations are defined in Table 6. (cont.)

\begin{tabular}{|c|c|c|c|c|c|c|c|c|c|c|c|c|c|c|c|c|c|}
\hline \multirow[b]{2}{*}{ Reference } & \multirow[b]{2}{*}{$\begin{array}{c}\text { Test } \\
\#\end{array}$} & \multicolumn{5}{|c|}{$\begin{array}{c}\text { Recovery concentration results - } \\
\text { Mean \& \%RSDs } \\
\end{array}$} & \multicolumn{5}{|c|}{$\begin{array}{l}\text { Recovery efficiency (RE) - } \\
\text { Mean \& \%RSDs } \\
\end{array}$} & \multicolumn{6}{|c|}{ LOD, FNR, and FPR } \\
\hline & & $\begin{array}{c}\text { Mean } \\
\left(\mathrm{CFU} / \mathrm{cm}^{2}\right)\end{array}$ & $\begin{array}{l}\text { Lab } \\
\% \text { RSD }\end{array}$ & $\begin{array}{c}\text { Run } \\
\% \text { RSD }\end{array}$ & $\begin{array}{c}\text { Sample- } \\
\text { within-run } \\
\% \text { RSD }\end{array}$ & $\begin{array}{c}\text { Total } \\
\% \text { RSD }\end{array}$ & $\begin{array}{c}\mathrm{RE} \\
\text { mean } \\
(\%) \\
\end{array}$ & $\begin{array}{l}\text { RE lab } \\
\text { \%RSD }\end{array}$ & $\begin{array}{l}\text { RE run } \\
\% \text { RSD }\end{array}$ & $\begin{array}{c}\text { RE sample- } \\
\text { within-run } \\
\% \text { RSD }\end{array}$ & $\begin{array}{c}\mathrm{RE} \\
\text { total } \\
\% \mathrm{RSD}\end{array}$ & $\begin{array}{c}\text { Positive } \\
\text { result } \\
(\mathrm{CFU})\end{array}$ & $\begin{array}{l}\text { LOD } \\
\text { defin- } \\
\text { ition }\end{array}$ & $\begin{array}{c}\text { LOD } \\
\left(\mathrm{CFU} / \mathrm{cm}^{2}\right)\end{array}$ & $\begin{array}{l}\text { LOD SD } \\
\text { or } 95 \% \mathrm{CI} \\
\left(\mathrm{CFU} / \mathrm{cm}^{2}\right)\end{array}$ & FNR & FPR \\
\hline \multirow{20}{*}{$\begin{array}{l}\text { Edmonds } \\
\text { et al. } 2009 \\
\text { (cont.) }\end{array}$} & 6.17 & NR & NA & NR & NR & NA & 62.4 & NA & 16 & 13 & NA & NR & NR & NR & NR & NR & NR \\
\hline & 6.18 & NR & NA & NR & NR & NA & 64.9 & NA & 14 & 10 & NA & NR & NR & NR & NR & NR & NR \\
\hline & 6.19 & NR & NA & NR & NR & NA & 65.2 & NA & 23 & 17 & NA & NR & NR & NR & NR & NR & NR \\
\hline & 6.20 & NR & NA & NR & NR & NA & 61.2 & NA & 20 & 13 & NA & NR & NR & NR & NR & NR & NR \\
\hline & 6.21 & NR & NA & NR & NR & NA & 51.9 & NA & 35 & 11 & NA & NR & NR & NR & NR & NR & NR \\
\hline & 6.22 & NR & NA & NR & NR & NA & 57.6 & NA & 27 & 8 & NA & NR & NR & NR & NR & NR & NR \\
\hline & 6.23 & NR & NA & NR & NR & NA & 53.1 & NA & 33 & 9 & NA & NR & NR & NR & NR & NR & NR \\
\hline & 6.24 & NR & NA & NR & NR & NA & 51.5 & NA & 32 & 10 & NA & NR & NR & NR & NR & NR & NR \\
\hline & 6.25 & NR & NA & NR & NR & NA & 65.1 & NA & 16 & 14 & NA & NR & NR & NR & NR & NR & NR \\
\hline & 6.26 & NR & NA & NR & NR & NA & 71.9 & NA & 10 & 10 & NA & NR & NR & NR & NR & NR & NR \\
\hline & 6.27 & NR & NA & NR & NR & NA & 68.9 & NA & 12 & 10 & NA & NR & NR & NR & NR & NR & NR \\
\hline & 6.28 & NR & NA & NR & NR & NA & 75.5 & NA & 10 & 11 & NA & NR & NR & NR & NR & NR & NR \\
\hline & 6.29 & NR & NA & NR & NR & NA & 60.3 & NA & 12 & 10 & NA & NR & NR & NR & NR & NR & NR \\
\hline & 6.30 & NR & NA & NR & NR & NA & 68.7 & NA & 16 & 10 & NA & NR & NR & NR & NR & NR & NR \\
\hline & 6.31 & NR & NA & NR & NR & NA & 60.2 & NA & 17 & 20 & NA & NR & NR & NR & NR & NR & NR \\
\hline & 6.32 & NR & NA & NR & NR & NA & 67.0 & NA & 14 & 13 & NA & NR & NR & NR & NR & NR & NR \\
\hline & 6.33 & 2010 & NA & $25^{\text {(a) }}$ & (a) & NA & 42.1 & NA & NR & 25 & NA & NR & NR & NR & NR & NR & NR \\
\hline & 6.34 & 18400 & NA & $29^{(\mathrm{a})}$ & (a) & NA & 61.1 & NA & NR & 29 & NA & NR & NR & NR & NR & NR & NR \\
\hline & 6.35 & 221000 & NA & $19^{(\mathrm{a})}$ & (a) & NA & 75.8 & NA & NR & 19 & NA & NR & NR & NR & NR & NR & NR \\
\hline & 6.36 & $2.36 \mathrm{E}+6$ & NA & $26^{(\mathrm{a})}$ & (a) & NA & 92.7 & NA & NR & 27 & NA & NR & NR & NR & NR & NR & NR \\
\hline \multirow{17}{*}{$\begin{array}{l}\text { Valentine } \\
\text { et al. } 2008\end{array}$} & 7.1 & NR & NA & NR & NR & NA & 5.1 & NA & NR & 43 & NA & NR & NR & $\mathrm{NR}$ & NR & $\mathrm{NR}$ & NR \\
\hline & 7.2 & NR & NA & NR & NR & NA & 6.6 & NA & NR & 33 & NA & NR & NR & NR & NR & NR & NR \\
\hline & 7.3 & NR & NA & NR & NR & NA & 3.3 & NA & NR & 25 & NA & NR & NR & NR & NR & NR & NR \\
\hline & 7.4 & NR & NA & NR & NR & NA & 1.9 & NA & NR & 55 & NA & NR & NR & NR & NR & NR & NR \\
\hline & 7.5 & NR & NA & NR & NR & NA & 3.9 & NA & NR & 31 & NA & NR & NR & NR & NR & NR & NR \\
\hline & 7.6 & NR & NA & NR & NR & NA & 4.2 & NA & NR & 28 & NA & NR & NR & NR & NR & NR & NR \\
\hline & 7.7 & NR & NA & NR & NR & NA & 2.3 & NA & NR & 33 & NA & NR & NR & NR & NR & NR & NR \\
\hline & 7.8 & NR & NA & NR & NR & NA & 1.7 & NA & NR & 37 & NA & NR & NR & NR & NR & NR & NR \\
\hline & 7.9 & NR & NA & NR & NR & NA & 2.9 & NA & NR & 87 & NA & NR & NR & NR & NR & NR & NR \\
\hline & 7.10 & NR & NA & NR & NR & NA & 3.9 & NA & NR & 40 & NA & NR & NR & NR & NR & NR & NR \\
\hline & 7.11 & NR & NA & NR & NR & NA & 1.7 & NA & NR & 41 & NA & NR & NR & NR & NR & NR & NR \\
\hline & 7.12 & NR & NA & NR & NR & NA & 1.3 & NA & NR & 32 & NA & NR & NR & NR & NR & NR & NR \\
\hline & 7.13 & NR & NA & NR & NR & NA & 0.6 & NA & NR & 58 & NA & NR & NR & NR & NR & NR & NR \\
\hline & 7.14 & NR & NA & NR & NR & NA & 0.9 & NA & NR & 39 & NA & NR & NR & NR & NR & NR & NR \\
\hline & 7.15 & NR & NA & NR & NR & NA & 1.4 & NA & NR & 150 & NA & NR & NR & NR & NR & NR & NR \\
\hline & 7.16 & NR & NA & NR & NR & NA & 0.6 & NA & NR & 35 & NA & NR & NR & NR & NR & NR & NR \\
\hline & 7.17 & NR & NA & NR & NR & NA & 0.5 & NA & NR & 84 & NA & NR & NR & NR & NR & NR & NR \\
\hline
\end{tabular}


Table A.1b Expanded summary of test results for swab sampling. Acronyms and abbreviations are defined in Table 6. (cont.)

\begin{tabular}{|c|c|c|c|c|c|c|c|c|c|c|c|c|c|c|c|c|c|}
\hline \multirow[b]{2}{*}{ Reference } & \multirow[b]{2}{*}{$\begin{array}{c}\text { Test } \\
\#\end{array}$} & \multicolumn{5}{|c|}{$\begin{array}{c}\text { Recovery concentration results - } \\
\text { Mean \& \%RSDs } \\
\end{array}$} & \multicolumn{5}{|c|}{$\begin{array}{l}\text { Recovery efficiency (RE) - } \\
\text { Mean \& \%RSDs } \\
\end{array}$} & \multicolumn{6}{|c|}{ LOD, FNR, and FPR } \\
\hline & & $\begin{array}{c}\text { Mean } \\
\left(\mathrm{CFU} / \mathrm{cm}^{2}\right)\end{array}$ & $\begin{array}{l}\text { Lab } \\
\% \text { RSD }\end{array}$ & $\begin{array}{l}\text { Run } \\
\% \text { RSD }\end{array}$ & $\begin{array}{c}\text { Sample- } \\
\text { within-run } \\
\% \text { RSD }\end{array}$ & $\begin{array}{c}\text { Total } \\
\% \text { RSD }\end{array}$ & \begin{tabular}{|c|}
$\mathrm{RE}$ \\
mean \\
$(\%)$ \\
\end{tabular} & $\begin{array}{l}\text { RE lab } \\
\text { \%RSD }\end{array}$ & $\begin{array}{l}\text { RE run } \\
\% \mathrm{RSD}\end{array}$ & $\begin{array}{c}\text { RE sample- } \\
\text { within-run } \\
\% \mathrm{RSD}\end{array}$ & \begin{tabular}{|c|}
$\mathrm{RE}$ \\
total \\
$\% \mathrm{RSD}$ \\
\end{tabular} & $\begin{array}{c}\text { Positive } \\
\text { result } \\
(\mathrm{CFU})\end{array}$ & $\begin{array}{l}\text { LOD } \\
\text { defin- } \\
\text { ition }\end{array}$ & $\begin{array}{c}\text { LOD } \\
\left(\mathrm{CFU} / \mathrm{cm}^{2}\right)\end{array}$ & $\begin{array}{c}\text { LOD SD } \\
\text { or } 95 \% \text { CI } \\
\left(\mathrm{CFU} / \mathrm{cm}^{2}\right) \\
\end{array}$ & FNR & FPR \\
\hline \multirow{3}{*}{$\begin{array}{l}\text { Valentine } \\
\text { et al. } 2008 \\
\text { (cont.) }\end{array}$} & 7.18 & NR & NA & NR & NR & NA & 2.7 & NA & NR & 18 & NA & $\mathrm{NR}$ & NR & $\mathrm{NR}$ & NR & NR & NR \\
\hline & 7.19 & NR & NA & NR & NR & NA & 0.8 & NA & NR & 35 & NA & NR & NR & NR & NR & NR & NR \\
\hline & 7.20 & NR & NA & NR & NR & NA & 0.8 & NA & NR & 26 & NA & NR & NR & NR & NR & NR & NR \\
\hline \multirow{18}{*}{$\begin{array}{l}\text { Buttner } \\
\text { et al. } 2001\end{array}$} & 8.1 & NR & NA & NR & NR & NA & 73.5 & NA & NR & 7 & NA & NR & NR & NR & NR & NR & NR \\
\hline & 8.2 & NR & NA & NR & NR & NA & 68.6 & NA & NR & 6 & NA & NR & NR & NR & NR & NR & NR \\
\hline & 8.3 & 748 & NA & $43^{(a)}$ & (a) & NA & NR & NA & NR & NR & NA & NR & NR & NR & NR & NR & NR \\
\hline & 8.4 & 149 & NA & $8^{(\mathrm{a})}$ & (a) & NA & NR & NA & NR & NR & NA & NR & NR & NR & NR & NR & NR \\
\hline & 8.5 & 103 & NA & $63^{(\mathrm{a})}$ & (a) & NA & NR & NA & NR & NR & NA & NR & NR & NR & NR & NR & NR \\
\hline & 8.6 & 226 & NA & $7^{(\mathrm{a})}$ & (a) & NA & NR & NA & NR & NR & NA & NR & NR & NR & NR & NR & NR \\
\hline & 8.7 & 544 & NA & $44^{(\mathrm{a})}$ & (a) & NA & NR & NA & NR & NR & NA & NR & NR & NR & NR & NR & NR \\
\hline & 8.8 & 93 & NA & $71^{(\mathrm{a})}$ & (a) & NA & NR & NA & NR & NR & NA & NR & NR & NR & NR & NR & NR \\
\hline & 8.9 & 82 & NA & $107^{(\mathrm{a})}$ & (a) & NA & NR & NA & NR & NR & NA & NR & NR & NR & NR & NR & NR \\
\hline & 8.10 & 101 & NA & $82^{(\mathrm{a})}$ & (a) & NA & NR & NA & NR & NR & NA & NR & NR & NR & NR & NR & NR \\
\hline & 8.11 & 706 & NA & $25^{(\mathrm{a})}$ & (a) & NA & NR & NA & NR & NR & NA & NR & NR & NR & NR & NR & NR \\
\hline & 8.12 & 164 & NA & $23^{(\mathrm{a})}$ & (a) & NA & NR & NA & NR & NR & NA & NR & NR & NR & NR & NR & NR \\
\hline & 8.13 & 156 & NA & $44^{(\mathrm{a})}$ & (a) & NA & NR & NA & NR & NR & NA & NR & NR & NR & NR & NR & NR \\
\hline & 8.14 & 296 & NA & $18^{(\mathrm{a})}$ & (a) & NA & NR & NA & NR & NR & NA & NR & NR & NR & NR & NR & NR \\
\hline & 8.15 & 1264 & NA & $85^{(\mathrm{a})}$ & (a) & NA & NR & NA & NR & NR & NA & NR & NR & NR & NR & NR & NR \\
\hline & 8.16 & 122 & NA & $23^{(\mathrm{a})}$ & (a) & NA & NR & NA & NR & NR & NA & NR & NR & NR & NR & NR & NR \\
\hline & 8.17 & 84 & NA & $47^{(\mathrm{a})}$ & (a) & NA & NR & NA & NR & NR & NA & NR & NR & NR & NR & NR & NR \\
\hline & 8.18 & 294 & NA & $76^{(\mathrm{a})}$ & (a) & NA & NR & NA & NR & NR & NA & NR & NR & NR & NR & NR & NR \\
\hline \multirow{9}{*}{$\begin{array}{l}\text { Buttner et } \\
\text { al. 2004a }\end{array}$} & 9.1 & 492 & NA & $92^{(\mathrm{a})}$ & (a) & NA & NR & NA & NR & NR & NA & NR & NR & NR & NR & NR & NR \\
\hline & 9.2 & 340 & NA & $44^{(\mathrm{a})}$ & (a) & NA & NR & NA & NR & NR & NA & NR & NR & NR & NR & NR & NR \\
\hline & 9.3 & 492 & NA & $68^{(\mathrm{a})}$ & (a) & NA & NR & NA & NR & NR & NA & NR & NR & NR & NR & NR & NR \\
\hline & 9.4 & 552 & NA & $24^{\text {(a) }}$ & (a) & NA & NR & NA & NR & NR & NA & NR & NR & NR & NR & NR & NR \\
\hline & 9.5 & 711 & NA & $48^{(\mathrm{a})}$ & (a) & NA & NR & NA & NR & NR & NA & NR & NR & NR & NR & NR & NR \\
\hline & 9.6 & 481 & NA & $56^{(\mathrm{a})}$ & (a) & NA & NR & NA & NR & NR & NA & NR & NR & NR & NR & NR & NR \\
\hline & 9.7 & $523^{(\text {b) }}$ & NA & NR & $9^{(b)}$ & NA & NR & NA & NR & NR & NA & NR & NR & NR & NR & NR & NR \\
\hline & 9.8 & $704^{(\mathrm{b})}$ & NA & NR & $55^{(\mathrm{b})}$ & NA & NR & NA & NR & NR & NA & NR & NR & NR & NR & NR & NR \\
\hline & 9.9 & $594^{(\mathrm{b})}$ & NA & NR & $8^{(\mathrm{b})}$ & NA & NR & NA & NR & NR & NA & NR & NR & NR & NR & NR & NR \\
\hline \multirow{4}{*}{$\begin{array}{l}\text { Buttner et } \\
\text { al. } 2004 \mathrm{~b}\end{array}$} & 10.1 & 840 & NA & NR & 11 & NA & NR & NA & NR & NR & NA & NR & NR & NR & NR & NR & NR \\
\hline & 10.2 & 1200 & NA & NR & 22 & NA & NR & NA & NR & NR & NA & NR & NR & NR & NR & NR & NR \\
\hline & 10.3 & 1030 & NA & NR & NR & NA & NR & NA & NR & NR & NA & NR & NR & NR & NR & NR & NR \\
\hline & 10.4 & 1180 & NA & NR & 28 & NA & NR & NA & NR & NR & NA & NR & NR & NR & NR & NR & NR \\
\hline \multirow{4}{*}{$\begin{array}{l}\text { Quizon et } \\
\text { al. } 2007\end{array}$} & 11.1 & 5500 & NA & $27^{(\mathrm{a})}$ & (a) & NA & NR & NA & NR & NR & NA & NR & NR & NR & NR & NR & NR \\
\hline & 11.2 & 5110 & NA & $17^{(\mathrm{a})}$ & (a) & NA & 9.8 & NA & (c) & (c) & NA & NR & NR & NR & NR & NR & NR \\
\hline & 11.3 & 4170 & NA & $36^{\text {(a) }}$ & (a) & NA & NR & NA & NR & NR & NA & NR & NR & NR & NR & NR & NR \\
\hline & 11.4 & 5670 & NA & $19^{(\mathrm{a})}$ & (a) & NA & NR & NA & NR & NR & NA & NR & NR & NR & NR & NR & NR \\
\hline
\end{tabular}


Table A.1b Expanded summary of test results for swab sampling. Acronyms and abbreviations are defined in Table 6. (cont.)

\begin{tabular}{|c|c|c|c|c|c|c|c|c|c|c|c|c|c|c|c|c|c|}
\hline \multirow[b]{2}{*}{ Reference } & \multirow[b]{2}{*}{$\begin{array}{c}\text { Test } \\
\#\end{array}$} & \multicolumn{5}{|c|}{$\begin{array}{c}\text { Recovery concentration results - } \\
\text { Mean \& \%RSDs } \\
\end{array}$} & \multicolumn{5}{|c|}{$\begin{array}{l}\text { Recovery efficiency (RE) - } \\
\text { Mean \& \%RSDs } \\
\end{array}$} & \multicolumn{6}{|c|}{ LOD, FNR, and FPR } \\
\hline & & $\begin{array}{c}\text { Mean } \\
\left(\mathrm{CFU} / \mathrm{cm}^{2}\right)\end{array}$ & $\begin{array}{l}\text { Lab } \\
\% \text { RSD }\end{array}$ & $\begin{array}{l}\text { Run } \\
\% \text { RSD }\end{array}$ & $\begin{array}{c}\text { Sample- } \\
\text { within-run } \\
\% \mathrm{RSD}\end{array}$ & $\begin{array}{c}\text { Total } \\
\% \text { RSD }\end{array}$ & \begin{tabular}{|c}
$\mathrm{RE}$ \\
mean \\
$(\%)$ \\
\end{tabular} & $\begin{array}{l}\text { RE lab } \\
\% \text { RSD }\end{array}$ & $\begin{array}{l}\text { RE run } \\
\% \text { RSD }\end{array}$ & $\begin{array}{c}\text { RE sample- } \\
\text { within-run } \\
\% \mathrm{RSD} \\
\end{array}$ & \begin{tabular}{|c|}
$\mathrm{RE}$ \\
total \\
$\% \mathrm{RSD}$ \\
\end{tabular} & $\begin{array}{c}\text { Positive } \\
\text { result } \\
(\mathrm{CFU})\end{array}$ & $\begin{array}{l}\text { LOD } \\
\text { defin- } \\
\text { ition }\end{array}$ & $\begin{array}{c}\mathrm{LOD} \\
\left(\mathrm{CFU} / \mathrm{cm}^{2}\right)\end{array}$ & $\begin{array}{c}\text { LOD SD } \\
\text { or } 95 \% \text { CI } \\
\left(\mathrm{CFU} / \mathrm{cm}^{2}\right)\end{array}$ & FNR & FPR \\
\hline \multirow{22}{*}{$\begin{array}{l}\text { Nellen et } \\
\text { al. } 2006\end{array}$} & 12.1 & 0.29 & NA & $17^{(\mathrm{a})}$ & (a) & NA & 13.4 & NA & $17^{(\mathrm{a})}$ & (a) & NA & $\mathrm{NR}$ & NR & $\mathrm{NR}$ & $\mathrm{NR}$ & $\mathrm{NR}$ & NR \\
\hline & 12.2 & 0.29 & NA & $17^{(\mathrm{a})}$ & (a) & NA & 13.4 & NA & $18^{(\mathrm{a})}$ & (a) & NA & NR & NR & NR & NR & NR & NR \\
\hline & 12.3 & 1.90 & NA & $3^{(a)}$ & (a) & NA & 88.0 & NA & $4^{(a)}$ & (a) & NA & NR & NR & NR & NR & NR & NR \\
\hline & 12.4 & 1.99 & NA & $1^{\text {(a) }}$ & (a) & NA & 92.2 & NA & $3^{(\mathrm{a})}$ & (a) & NA & NR & NR & NR & NR & NR & NR \\
\hline & 12.5 & 1.57 & NA & $4^{(a)}$ & (a) & NA & 72.7 & NA & $5^{(\mathrm{a})}$ & (a) & NA & NR & NR & NR & NR & NR & NR \\
\hline & 12.6 & 1.42 & NA & $6^{(\mathrm{a})}$ & (a) & NA & 65.8 & NA & $7^{(\mathrm{a})}$ & (a) & NA & NR & NR & NR & NR & NR & NR \\
\hline & 12.7 & 2.04 & NA & $1^{\text {(a) }}$ & (a) & NA & 94.5 & NA & $3^{(\mathrm{a})}$ & (a) & NA & NR & NR & NR & NR & NR & NR \\
\hline & 12.8 & 2.02 & NA & $4^{(\mathrm{a})}$ & (a) & NA & 93.6 & NA & $4^{(\mathrm{a})}$ & (a) & NA & NR & NR & NR & NR & NR & NR \\
\hline & 12.9 & 1.75 & NA & $31^{\text {(a) }}$ & (a) & NA & 66.1 & NA & $33^{(\mathrm{a})}$ & (a) & NA & NR & NR & NR & NR & NR & NR \\
\hline & 12.10 & 1.56 & NA & $30^{(\mathrm{a})}$ & (a) & NA & 58.9 & NA & $31^{\text {(a) }}$ & (a) & NA & NR & NR & NR & NR & NR & NR \\
\hline & 12.11 & 1.22 & NA & $25^{(\mathrm{a})}$ & (a) & NA & 46.1 & NA & $27^{(\mathrm{a})}$ & (a) & NA & NR & NR & NR & NR & NR & NR \\
\hline & 12.12 & 0.95 & NA & $33^{(\mathrm{a})}$ & (a) & NA & 35.9 & NA & $34^{(\mathrm{a})}$ & (a) & NA & NR & NR & NR & NR & NR & NR \\
\hline & 12.13 & 2.61 & NA & $27^{(\mathrm{a})}$ & (a) & NA & 64.1 & NA & $31^{\text {(a) }}$ & (a) & NA & NR & NR & NR & NR & NR & NR \\
\hline & 12.14 & 2.13 & NA & $25^{(\mathrm{a})}$ & (a) & NA & 55.9 & NA & $27^{(\mathrm{a})}$ & (a) & NA & NR & NR & NR & NR & NR & NR \\
\hline & 12.15 & 1.71 & NA & $40^{(\mathrm{a})}$ & (a) & NA & 44.6 & NA & $43^{(\mathrm{a})}$ & (a) & NA & NR & NR & NR & NR & NR & NR \\
\hline & 12.16 & 2.72 & NA & $24^{(\mathrm{a})}$ & (a) & NA & 73.7 & NA & $26^{(\mathrm{a})}$ & (a) & NA & NR & NR & NR & NR & NR & NR \\
\hline & 12.17 & 1.92 & NA & $56^{(\mathrm{a})}$ & (a) & NA & 53.6 & NA & $52^{\text {(a) }}$ & (a) & NA & NR & NR & NR & NR & NR & NR \\
\hline & 12.18 & 1.12 & NA & $47^{(\mathrm{a})}$ & (a) & NA & 28.1 & NA & $47^{(\mathrm{a})}$ & (a) & NA & NR & NR & NR & NR & NR & NR \\
\hline & 12.19 & 0.73 & NA & $52^{(\mathrm{a})}$ & (a) & NA & 19.8 & NA & $48^{(\mathrm{a})}$ & (a) & NA & NR & NR & NR & NR & NR & NR \\
\hline & 12.20 & 0.93 & NA & $52^{(\mathrm{a})}$ & (a) & NA & 24.9 & NA & $50^{(\mathrm{a})}$ & (a) & NA & NR & NR & NR & NR & NR & NR \\
\hline & 12.21 & 0.99 & NA & $54^{(\mathrm{a})}$ & (a) & NA & 27.9 & NA & $49^{(\mathrm{a})}$ & (a) & NA & NR & NR & NR & NR & NR & NR \\
\hline & 12.22 & 1.07 & NA & $35^{(\mathrm{a})}$ & (a) & NA & 28.6 & NA & $38^{(\mathrm{a})}$ & (a) & NA & NR & NR & NR & NR & NR & NR \\
\hline \multirow{12}{*}{$\begin{array}{l}\text { Hodges et } \\
\text { al. } 2010\end{array}$} & \begin{tabular}{|l|}
13.1 \\
\end{tabular} & 0.48 & NR & NR & $\mathrm{NR}$ & NR & 25.7 & 15.6 & (d) & $57^{(\mathrm{d})}$ & 59.0 & $\geq 1 \mathrm{CFU} / \mathrm{ml}$ & & & & 0.017 & NR \\
\hline & 13.2 & 3.07 & NR & NR & NR & NR & 15.8 & 24.2 & (d) & $34^{(\mathrm{d})}$ & 41.6 & $\geq 1 \mathrm{CFU} / \mathrm{ml}$ & NR & 0.8 & NR & 0 & NR \\
\hline & 13.3 & 498.22 & NR & NR & NR & NR & 31.0 & 21.1 & (d) & $28^{(\mathrm{d})}$ & 35.0 & $\geq 1 \mathrm{CFU} / \mathrm{ml}$ & & & & 0 & NR \\
\hline & 13.4 & 0.99 & NR & NR & NR & NR & 61.0 & 18.2 & (d) & $32^{(\mathrm{d})}$ & 37.6 & $\geq 1 \mathrm{CFU} / \mathrm{ml}$ & NR & NR & NR & 0 & 0.083 \\
\hline & 13.5 & 9.05 & NR & NR & NR & NR & 63.0 & 12.1 & (d) & $10^{(\mathrm{d})}$ & 15.4 & $\geq 1 \mathrm{CFU} / \mathrm{ml}$ & NR & NR & NR & 0 & $\begin{array}{c}0.000 \\
0\end{array}$ \\
\hline & 13.6 & 1064.32 & NR & NR & NR & NR & 83.1 & 19.6 & (d) & $9^{\text {(d) }}$ & 21.7 & $\geq 1 \mathrm{CFU} / \mathrm{ml}$ & NR & NR & NR & 0 & 0 \\
\hline & 13.7 & 0.76 & NR & NR & NR & NR & 55.0 & 30.3 & (d) & $40^{(\mathrm{d})}$ & 50.2 & $\geq 1 \mathrm{CFU} / \mathrm{ml}$ & NR & NR & NR & 0.017 & NR \\
\hline & 13.8 & 4.26 & NR & NR & NR & NR & 27.9 & 46.8 & (d) & $33^{(\mathrm{d})}$ & 57.1 & $\geq 1 \mathrm{CFU} / \mathrm{ml}$ & NR & NR & NR & 0 & NR \\
\hline & 13.9 & 499.15 & NR & NR & NR & NR & 42.0 & 37.2 & (d) & $24^{(\mathrm{d})}$ & 44.3 & $\geq 1 \mathrm{CFU} / \mathrm{ml}$ & NR & NR & NR & 0 & NR \\
\hline & 13.10 & 0.71 & NR & NR & NR & NR & 51.6 & 36.8 & (d) & $41^{(\mathrm{d})}$ & 54.9 & $\geq 1 \mathrm{CFU} / \mathrm{ml}$ & NR & NR & NR & 0 & 0.146 \\
\hline & 13.11 & 5.47 & NR & NR & NR & NR & 35.8 & 45.4 & (d) & $15^{(\mathrm{d})}$ & 47.8 & $\geq 1 \mathrm{CFU} / \mathrm{ml}$ & NR & NR & NR & 0 & 0.104 \\
\hline & 13.12 & 878.27 & NR & NR & NR & NR & 73.9 & 26.7 & (d) & $18^{(\mathrm{d})}$ & 32.0 & $\geq 1 \mathrm{CFU} / \mathrm{ml}$ & NR & NR & NR & 0 & 0 \\
\hline
\end{tabular}

(a) Combined estimate of "run" and "sample-within-run" uncertainties.

(b) Mean and sample-within-run \%RSD were calculated treating pre- and post- results in Table 1 of Buttner et al. (2004a) as replicate tests.

(c) Quizon et al. (2007) reported SE = 1.9; insufficient info to 1) know whether it includes run-to-run and/or sample-within-run uncertainties, and 2) convert the SE to a \%RSD.

(d) The precision (variance within laboratories) reported by Hodges et al. (2010) is listed in this table as "sample-within-run" uncertainty. 
Table A.2a Expanded summary of test conditions for wipe sampling. Acronyms and abbreviations are defined in Table 6.

\begin{tabular}{|c|c|c|c|c|c|c|c|c|c|c|c|c|c|c|c|}
\hline Reference & $\begin{array}{c}\text { Test } \\
\#\end{array}$ & Agent & $\begin{array}{l}\text { Agent } \\
\text { deposition }\end{array}$ & $\begin{array}{c}\text { Agent } \\
\text { concentration } \\
\left(\mathrm{CFU} / \mathrm{cm}^{2}\right)\end{array}$ & Wipe type & $\begin{array}{c}\text { Wetting } \\
\text { agent }\end{array}$ & $\begin{array}{c}\text { Relative } \\
\text { humidity } \\
(\%)\end{array}$ & $\begin{array}{c}\text { Surface } \\
\text { type }\end{array}$ & $\begin{array}{l}\text { Surface } \\
\text { area } \\
\text { sampled } \\
\left(\mathrm{cm}^{2}\right) \\
\end{array}$ & $\begin{array}{c}\text { Extraction } \\
\text { liquid }\end{array}$ & $\begin{array}{c}\text { Extraction } \\
\text { method }\end{array}$ & $\begin{array}{l}\text { Culture } \\
\text { method, } \\
\text { medium }\end{array}$ & $\begin{array}{c}\# \\
\text { labs } \\
\end{array}$ & $\begin{array}{c}\# \text { test } \\
\text { runs }\end{array}$ & $\begin{array}{c}\text { Total } \\
\text { \# test } \\
\text { samples } \\
\end{array}$ \\
\hline Estill et & 1.1 & BA Sterne & Dry aerosol & 0.03 & Sponge & BBT & NR & SS & 929 & BBT & $\mathrm{A}+\mathrm{C}+\mathrm{V}+\mathrm{S}$ & FP, TSAB & 3 & 3 & 27 \\
\hline \multirow[t]{5}{*}{ al. 2009} & 1.2 & BA Sterne & Dry aerosol & 0.3 & Sponge & $\mathrm{BBT}$ & NR & SS & 929 & BBT & $\mathrm{A}+\mathrm{C}+\mathrm{V}+\mathrm{S}$ & FP, TSAB & 3 & 3 & 27 \\
\hline & 1.3 & BA Sterne & Dry aerosol & 2 & Sponge & BBT & NR & SS & 929 & BBT & $\mathrm{A}+\mathrm{C}+\mathrm{V}+\mathrm{S}$ & FP, TSAB & 3 & 3 & 27 \\
\hline & 1.4 & BA Sterne & Dry aerosol & 0.03 & Sponge & BBT & NR & Carpet & 929 & BBT & $\mathrm{A}+\mathrm{C}+\mathrm{V}+\mathrm{S}$ & FP, TSAB & 3 & 2 & 18 \\
\hline & 1.5 & BA Sterne & Dry aerosol & 0.3 & Sponge & BBT & NR & Carpet & 929 & BBT & $\mathrm{A}+\mathrm{C}+\mathrm{V}+\mathrm{S}$ & FP, TSAB & 3 & 4 & 36 \\
\hline & 1.6 & BA Sterne & Dry aerosol & 2 & Sponge & BBT & NR & Carpet & 929 & BBT & $\mathrm{A}+\mathrm{C}+\mathrm{V}+\mathrm{S}$ & FP, TSAB & 3 & 3 & 26 \\
\hline Brown et & 2.1 & $\mathrm{BA}$ & Dry aerosol & $1 \mathrm{E}+2-1 \mathrm{E}+3$ & PR gauze & DW & NR & SS & 25 & BBT & $\mathrm{S}+\mathrm{H}+\mathrm{V}$ & Plate, PF & 1 & NR & 20 \\
\hline \multirow[t]{5}{*}{ al. $2007 \mathrm{a}$} & 2.2 & BA & Dry aerosol & $1 \mathrm{E}+4-1 \mathrm{E}+5$ & PR gauze & DW & NR & SS & 25 & BBT & $\mathrm{S}+\mathrm{H}+\mathrm{V}$ & Plate, PF & 1 & NR & 20 \\
\hline & 2.3 & BA & Dry aerosol & $1 \mathrm{E}+2-1 \mathrm{E}+3$ & PR gauze & DW & NR & PWB & 25 & BBT & $\mathrm{S}+\mathrm{H}+\mathrm{V}$ & Plate, PF & 1 & NR & 20 \\
\hline & 2.4 & BA & Dry aerosol & $1 E+4-1 E+5$ & PR gauze & DW & NR & PWB & 25 & BBT & $\mathrm{S}+\mathrm{H}+\mathrm{V}$ & Plate, PF & 1 & NR & 20 \\
\hline & 2.5 & BA & Liquid imm. & NR & PR gauze, DI & DW & NR & None & NA & BBT & $\mathrm{S}+\mathrm{H}+\mathrm{V}$ & Plate, PF & 1 & NR & 40 \\
\hline & 2.6 & BA & Liquid drops & $2.0 \mathrm{E}+5$ & NA & NA & NR & SS, DI & 25 & BBT & $\mathrm{S}+\mathrm{H}$ & Plate, BHIA & 1 & NR & 24 \\
\hline \multirow{13}{*}{$\begin{array}{l}\text { Buttner et } \\
\text { al. } 2001\end{array}$} & 3.1 & $\mathrm{BS}$ & Liquid drops & $1.48 \mathrm{E}+7$ & Sponge & PBST & NR & Glass & 32.49 & PBST & HS & Plate, TSAC & 1 & 3 & 3 \\
\hline & 3.2 & BS & Dry aerosol & $1 E+2-1 E+3$ & Sponge & PBST & NR & Vinyl & 32.49 & PBST & HS & Plate, TSAC & 1 & 3 & 3 \\
\hline & 3.3 & $\mathrm{BS}$ & Dry aerosol & $1 E+2-1 E+3$ & Sponge & PBST & NR & Cpt-R & 32.49 & PBST & HS & Plate, TSAC & 1 & 3 & 3 \\
\hline & 3.4 & $\mathrm{BS}$ & Dry aerosol & $1 E+2-1 E+3$ & Sponge & PBST & NR & Cpt-S & 32.49 & PBST & HS & Plate, TSAC & 1 & 3 & 3 \\
\hline & 3.5 & $\mathrm{BS}$ & Dry aerosol & $1 E+2-1 E+3$ & Sponge & PBST & NR & Cpt-C & 32.49 & PBST & HS & Plate, TSAC & 1 & 3 & 3 \\
\hline & 3.6 & $\mathrm{BS}+\mathrm{PC} 2$ & Dry aerosol & $1 E+2-1 E+3$ & Sponge & PBST & NR & Vinyl & 32.49 & PBST & HS & Plate, TSAC & 1 & 2 & 2 \\
\hline & 3.7 & $\mathrm{BS}+\mathrm{PC} 2$ & Dry aerosol & $1 \mathrm{E}+2-1 \mathrm{E}+3$ & Sponge & PBST & NR & Cpt-R & 32.49 & PBST & HS & Plate, TSAC & 1 & 2 & 2 \\
\hline & 3.8 & $\mathrm{BS}+\mathrm{PC} 2$ & Dry aerosol & $1 \mathrm{E}+2-1 \mathrm{E}+3$ & Sponge & PBST & NR & Cpt-S & 32.49 & PBST & HS & Plate, TSAC & 1 & 2 & 2 \\
\hline & 3.9 & $\mathrm{BS}+\mathrm{PC} 2$ & Dry aerosol & $1 E+2-1 E+3$ & Sponge & PBST & NR & Cpt-C & 32.49 & PBST & HS & Plate, TSAC & 1 & 2 & 2 \\
\hline & 3.10 & $\mathrm{BS}+\mathrm{PC} 4$ & Dry aerosol & $1 E+2-1 E+3$ & Sponge & PBST & NR & Vinyl & 32.49 & PBST & HS & Plate, TSAC & 1 & 2 & 2 \\
\hline & 3.11 & $\mathrm{BS}+\mathrm{PC} 4$ & Dry aerosol & $1 E+2-1 E+3$ & Sponge & PBST & NR & Cpt-R & 32.49 & PBST & HS & Plate, TSAC & 1 & 2 & 2 \\
\hline & 3.12 & $\mathrm{BS}+\mathrm{PC} 4$ & Dry aerosol & $1 E+2-1 E+3$ & Sponge & PBST & NR & Cpt-S & 32.49 & PBST & HS & Plate, TSAC & 1 & 2 & 2 \\
\hline & 3.13 & $\mathrm{BS}+\mathrm{PC} 4$ & Dry aerosol & $1 E+2-1 E+3$ & Sponge & PBST & NR & Cpt-C & 32.49 & PBST & HS & Plate, TSAC & 1 & 2 & 2 \\
\hline \multirow{15}{*}{$\begin{array}{l}\text { Valentine } \\
\text { et al. } 2008\end{array}$} & 4.1 & BS & Liquid drops & 90349.9 & Cotton & PBST & NR & Plastic & 104.04 & PBST & $\mathrm{V}+\mathrm{C}$ & Plate, TSA & 1 & 10 & 10 \\
\hline & 4.2 & $\mathrm{BS}$ & Liquid drops & 90349.9 & HCW & PBST & NR & Plastic & 104.04 & PBST & $\mathrm{V}+\mathrm{C}$ & Plate, TSA & 1 & 10 & 10 \\
\hline & 4.3 & BS & Liquid drops & 90349.9 & PR & PBST & NR & Plastic & 104.04 & PBST & $\mathrm{V}+\mathrm{C}$ & Plate, TSA & 1 & 10 & 10 \\
\hline & 4.4 & BS & Liquid drops & 90349.9 & Cotton & PBST & NR & Oak & 104.04 & PBST & $\mathrm{V}+\mathrm{C}$ & Plate, TSA & 1 & 10 & 10 \\
\hline & 4.5 & $\mathrm{BS}$ & Liquid drops & 90349.9 & HCW & PBST & NR & Oak & 104.04 & PBST & $\mathrm{V}+\mathrm{C}$ & Plate, TSA & 1 & 10 & 10 \\
\hline & 4.6 & $\mathrm{BS}$ & Liquid drops & 90349.9 & PR & PBST & NR & Oak & 104.04 & PBST & $\mathrm{V}+\mathrm{C}$ & Plate, TSA & 1 & 10 & 10 \\
\hline & 4.7 & BS & Liquid drops & 282,000 & Cotton & PBST & NR & Monitor & 25 & PBST & $\mathrm{V}+\mathrm{C}$ & Plate, TSA & 1 & 10 & 10 \\
\hline & 4.8 & $\mathrm{BS}$ & Liquid drops & 282,000 & HCW & PBST & NR & Monitor & 25 & PBST & $\mathrm{V}+\mathrm{C}$ & Plate, TSA & 1 & 10 & 10 \\
\hline & 4.9 & BS & Liquid drops & 282,000 & PR & PBST & NR & Monitor & 25 & PBST & $\mathrm{V}+\mathrm{C}$ & Plate, TSA & 1 & 10 & 10 \\
\hline & 4.10 & BS & Liquid drops & 90349.9 & Cotton & PBST & NR & PEUF & 104.04 & PBST & $\mathrm{V}+\mathrm{C}$ & Plate, TSA & 1 & 10 & 10 \\
\hline & 4.11 & BS & Liquid drops & 90349.9 & HCW & PBST & NR & PEUF & 104.04 & PBST & $\mathrm{V}+\mathrm{C}$ & Plate, TSA & 1 & 10 & 10 \\
\hline & 4.12 & $\mathrm{BS}$ & Liquid drops & 90349.9 & PR & PBST & NR & PEUF & 104.04 & PBST & $\mathrm{V}+\mathrm{C}$ & Plate, TSA & 1 & 10 & 10 \\
\hline & 4.13 & BS & Liquid drops & 90349.9 & Cotton & PBST & NR & Carpet & 104.04 & PBST & $\mathrm{V}+\mathrm{C}$ & Plate, TSA & 1 & 10 & 10 \\
\hline & 4.14 & $\mathrm{BS}$ & Liquid drops & 90349.9 & HCW & PBST & NR & Carpet & 104.04 & PBST & $\mathrm{V}+\mathrm{C}$ & Plate, TSA & 1 & 10 & 10 \\
\hline & 4.15 & $\mathrm{BS}$ & Liquid drops & 90349.9 & PR & PBST & NR & Carpet & 104.04 & PBST & $\mathrm{V}+\mathrm{C}$ & Plate, TSA & 1 & 10 & 10 \\
\hline
\end{tabular}


Table A.2a Expanded summary of test conditions for wipe sampling. Acronyms and abbreviations are defined in Table 6. (cont.)

\begin{tabular}{|c|c|c|c|c|c|c|c|c|c|c|c|c|c|c|c|}
\hline Reference & $\begin{array}{c}\text { Test } \\
\#\end{array}$ & Agent & $\begin{array}{l}\text { Agent } \\
\text { deposition }\end{array}$ & $\begin{array}{c}\text { Agent } \\
\text { concentration } \\
\left(\mathrm{CFU} / \mathrm{cm}^{2}\right)\end{array}$ & Wipe type & $\begin{array}{c}\text { Wetting } \\
\text { agent }\end{array}$ & $\begin{array}{c}\text { Relative } \\
\text { humidity } \\
(\%)\end{array}$ & $\begin{array}{c}\text { Surface } \\
\text { type }\end{array}$ & $\begin{array}{l}\text { Surface } \\
\text { area } \\
\text { sampled } \\
\left(\mathrm{cm}^{2}\right) \\
\end{array}$ & $\begin{array}{c}\text { Extraction } \\
\text { liquid }\end{array}$ & $\begin{array}{c}\text { Extraction } \\
\text { method }\end{array}$ & $\begin{array}{l}\text { Culture } \\
\text { method, } \\
\text { medium }\end{array}$ & $\begin{array}{c}\# \\
\text { labs } \\
\end{array}$ & $\begin{array}{l}\# \text { test } \\
\text { runs }\end{array}$ & $\begin{array}{c}\text { Total } \\
\text { \# test } \\
\text { samples } \\
\end{array}$ \\
\hline \multirow{18}{*}{$\begin{array}{l}\text { Buttner et } \\
\text { al. } 2004 \mathrm{a}\end{array}$} & 5.1 & BAtr & Dry aerosol & $107.6-1076.4$ & Swipe & PBST & NR & Vinyl & 929 & NR & HM & Plate, TSA & 1 & 3 & 3 \\
\hline & 5.2 & BAtr & Dry aerosol & $107.6-1076.4$ & Swipe & PBST & NR & Wood & 929 & NR & HM & Plate, TSA & 1 & 3 & 3 \\
\hline & 5.3 & BAtr & Dry aerosol & $107.6-1076.4$ & Swipe & PBST & NR & Metal & 929 & NR & HM & Plate, TSA & 1 & 3 & 3 \\
\hline & 5.4 & BAtr & Dry aerosol & $107.6-1076.4$ & Heavy Wipe & PBST & NR & Vinyl & 929 & NR & HM & Plate, TSA & 1 & 3 & 3 \\
\hline & 5.5 & BAtr & Dry aerosol & $107.6-1076.4$ & Heavy Wipe & PBST & NR & Wood & 929 & NR & HM & Plate, TSA & 1 & 3 & 3 \\
\hline & 5.6 & BAtr & Dry aerosol & $107.6-1076.4$ & Heavy Wipe & PBST & NR & Metal & 929 & NR & HM & Plate, TSA & 1 & 3 & 3 \\
\hline & 5.7 & BAtr & Dry aerosol & $107.6-1076.4$ & Swipe & PBST & NR & Vinyl & 929 & NR & HM & Plate, TSA & 1 & 3 & 3 \\
\hline & 5.8 & BAtr & Dry aerosol & $107.6-1076.4$ & Swipe & PBST & NR & Wood & 929 & NR & HM & Plate, TSA & 1 & 3 & 3 \\
\hline & 5.9 & BAtr & Dry aerosol & $107.6-1076.4$ & Swipe & PBST & NR & Metal & 929 & NR & HM & Plate, TSA & 1 & 3 & 3 \\
\hline & 5.10 & BAtr & Dry aerosol & $107.6-1076.4$ & Heavy Wipe & PBST & NR & Vinyl & 929 & NR & HM & Plate, TSA & 1 & 3 & 3 \\
\hline & 5.11 & BAtr & Dry aerosol & $107.6-1076.4$ & Heavy Wipe & PBST & NR & Wood & 929 & NR & $\mathrm{HM}$ & Plate, TSA & 1 & 3 & 3 \\
\hline & 5.12 & BAtr & Dry aerosol & $107.6-1076.4$ & Heavy Wipe & PBST & NR & Metal & 929 & NR & $\mathrm{HM}$ & Plate, TSA & 1 & 3 & 3 \\
\hline & 5.13 & BAtr & Dry aerosol & $107.6-1076.4$ & Swipe & PBST & NR & Vinyl & 929 & NR & $\mathrm{HM}$ & Plate, TSA & 1 & 1 & 2 \\
\hline & 5.14 & BAtr & Dry aerosol & $107.6-1076.4$ & Swipe & PBST & NR & Wood & 929 & NR & HM & Plate, TSA & 1 & 1 & 2 \\
\hline & 5.15 & BAtr & Dry aerosol & $107.6-1076.4$ & Swipe & PBST & NR & Metal & 929 & NR & HM & Plate, TSA & 1 & 1 & 1 \\
\hline & 5.16 & BAtr & Dry aerosol & $107.6-1076.4$ & Heavy Wipe & PBST & NR & Vinyl & 929 & NR & HM & Plate, TSA & 1 & 1 & 2 \\
\hline & 5.17 & BAtr & Dry aerosol & $107.6-1076.4$ & Heavy Wipe & PBST & NR & Wood & 929 & NR & $\mathrm{HM}$ & Plate, TSA & 1 & 1 & 2 \\
\hline & 5.18 & BAtr & Dry aerosol & $107.6-1076.4$ & Heavy Wipe & PBST & NR & Metal & 929 & NR & HM & Plate, TSA & 1 & 1 & 2 \\
\hline \multirow{10}{*}{$\begin{array}{l}\text { Buttner et } \\
\text { al. } 2004 b\end{array}$} & 6.1 & BAtr & Liquid imm. & 18.2 & BiSKit & Dry & NR & Metal & 10,000 & PBST & BiSKit & Plate, TSA & 1 & NR & 8 \\
\hline & 6.2 & BAtr & Liquid imm. & 18.2 & BiSKit & PBST & NR & Metal & 10,000 & PBST & BiSKit & Plate, TSA & 1 & NR & 4 \\
\hline & 6.3 & BAtr & Liquid imm. & 10 & BiSKit & Dry & NR & Metal & 10,000 & PBST & BiSKit & Plate, TSA & 1 & NR & 8 \\
\hline & 6.4 & BAtr & Liquid imm. & 10 & BiSKit & PBST & NR & Metal & 10,000 & PBST & BiSKit & Plate, TSA & 1 & NR & 8 \\
\hline & 6.5 & BAtr & Dry aerosol & NR & BiSKit & Dry & NR & Metal & 10,000 & PBST & BiSKit & Plate, TSA & 1 & NR & 4 \\
\hline & 6.6 & BAtr & Dry aerosol & NR & BiSKit & Dry & NR & Wood & 10,000 & PBST & BiSKit & Plate, TSA & 1 & NR & 4 \\
\hline & 6.7 & BAtr & Dry aerosol & NR & BiSKit & PBST & NR & Metal & 10,000 & PBST & BiSKit & Plate, TSA & 1 & NR & 4 \\
\hline & 6.8 & BAtr & Dry aerosol & NR & BiSKit & PBST & NR & Wood & 10,000 & PBST & BiSKit & Plate, TSA & 1 & NR & 4 \\
\hline & 6.9 & BAtr & Dry aerosol & NR & BiSKit & NR & NR & Metal & 10,000 & PBST & BiSKit & Plate, TSA & 1 & NR & 4 \\
\hline & 6.10 & BAtr & Dry aerosol & NR & BiSKit & NR & NR & Wood & 10,000 & PBST & BiSKit & Plate, TSA & 1 & NR & 8 \\
\hline \multirow{6}{*}{$\begin{array}{l}\text { Frawley et } \\
\text { al. } 2008\end{array}$} & 7.1 & BA Sterne & Liquid drops & $1 \mathrm{E}+2-1 \mathrm{E}+5$ & Gauze wipes & Dry & NR & Plastic & 1 & PBST & $\mathrm{V}$ & Plate, SBA & 1 & NR & 12 \\
\hline & 7.2 & BA Sterne & Liquid drops & (a) & Gauze wipes & Dry & NR & Wood & NR & PBST & V & Plate, SBA & 1 & NR & 12 \\
\hline & 7.3 & BA Sterne & Liquid drops & (a) & Gauze wipes & Dry & NR & Cotton & NR & PBST & V & Plate, SBA & 1 & NR & 12 \\
\hline & 7.4 & BA Sterne & Liquid drops & $1 \mathrm{E}+2-1 \mathrm{E}+5$ & Gauze wipes & $\mathrm{PBST} / \mathrm{Tr}$ & NR & Plastic & 1 & PBST & V & Plate, SBA & 1 & NR & 24 \\
\hline & 7.5 & BA Sterne & Liquid drops & (a) & Gauze wipes & $\mathrm{PBST} / \mathrm{Tr}$ & NR & Wood & NR & PBST & $\mathrm{V}$ & Plate, SBA & 1 & NR & 24 \\
\hline & 7.6 & BA Sterne & Liquid drops & (a) & Gauze wipes & $\mathrm{PBST} / \mathrm{Tr}$ & NR & Cotton & NR & PBST & $\mathrm{V}$ & Plate, SBA & 1 & NR & 24 \\
\hline \multirow{4}{*}{$\begin{array}{l}\text { Einfeld et } \\
\text { al. } 2011\end{array}$} & 8.1 & BAtr + grime & Dry aerosol & $3.27 \mathrm{E}+4-3.78 \mathrm{E}+4$ & PR & DW & 15 & SS & 100 & BBT & $\mathrm{S}+\mathrm{H}+\mathrm{V}$ & Plate, $\mathrm{PF}$ & 1 & 3 & 36 \\
\hline & 8.2 & BAtr & Dry aerosol & $3.27 \mathrm{E}+4-3.78 \mathrm{E}+4$ & PR & DW & 15 & SS & 100 & BBT & $\mathrm{S}+\mathrm{H}+\mathrm{V}$ & Plate, PF & 1 & 3 & 34 \\
\hline & 8.3 & BAtr + grime & Dry aerosol & $3.66 \mathrm{E}+4-5.60 \mathrm{E}+4$ & PR & DW & $82-90$ & SS & 100 & BBT & $\mathrm{S}+\mathrm{H}+\mathrm{V}$ & Plate, PF & 1 & 3 & 28 \\
\hline & 8.4 & BAtr & Dry aerosol & $3.66 \mathrm{E}+4-5.60 \mathrm{E}+4$ & PR & DW & $82-90$ & SS & 100 & BBT & $\mathrm{S}+\mathrm{H}+\mathrm{V}$ & Plate, PF & 1 & 3 & 31 \\
\hline
\end{tabular}


Table A.2a Expanded summary of test conditions for wipe sampling. Acronyms and abbreviations are defined in Table 6. (cont.)

\begin{tabular}{|c|c|c|c|c|c|c|c|c|c|c|c|c|c|c|c|}
\hline Reference & $\begin{array}{c}\text { Test } \\
\#\end{array}$ & Agent & $\begin{array}{l}\text { Agent } \\
\text { deposition }\end{array}$ & $\begin{array}{c}\text { Agent } \\
\text { concentration } \\
\left(\mathrm{CFU} / \mathrm{cm}^{2}\right)\end{array}$ & Wipe type & $\begin{array}{c}\text { Wetting } \\
\text { agent }\end{array}$ & $\begin{array}{c}\text { Relative } \\
\text { humidity } \\
(\%)\end{array}$ & $\begin{array}{c}\text { Surface } \\
\text { type }\end{array}$ & $\begin{array}{c}\text { Surface } \\
\text { area } \\
\text { sampled } \\
\left(\mathrm{cm}^{2}\right) \\
\end{array}$ & $\begin{array}{c}\text { Extraction } \\
\text { liquid }\end{array}$ & $\begin{array}{c}\text { Extraction } \\
\text { method }\end{array}$ & $\begin{array}{l}\text { Culture } \\
\text { method, } \\
\text { medium }\end{array}$ & $\begin{array}{c}\# \\
\text { labs }\end{array}$ & $\begin{array}{c}\# \text { test } \\
\text { runs }\end{array}$ & $\begin{array}{c}\text { Total } \\
\text { \# test } \\
\text { samples } \\
\end{array}$ \\
\hline \multirow{6}{*}{$\begin{array}{l}\text { Einfeld et } \\
\text { al. } 2011 \\
\text { (cont.) }\end{array}$} & 8.5 & BAtr + grime & Dry aerosol & $1.83 \mathrm{E}+4-6.81 \mathrm{E}+4$ & PR & DW & 90 & Glass & 100 & BBT & $\mathrm{S}+\mathrm{H}+\mathrm{V}$ & Plate, PF & 1 & 2 & 24 \\
\hline & 8.6 & BAtr & Dry aerosol & $1.83 \mathrm{E}+4-6.81 \mathrm{E}+4$ & PR & DW & 90 & Glass & 100 & BBT & $\mathrm{S}+\mathrm{H}+\mathrm{V}$ & Plate, PF & 1 & 2 & 24 \\
\hline & 8.7 & BAtr + grime & Dry aerosol & $9.55 \mathrm{E}+0-6.74 \mathrm{E}+4$ & PR & DW & $10-15$ & Marble & 100 & BBT & $\mathrm{S}+\mathrm{H}+\mathrm{V}$ & Plate, PF & 1 & 5 & 58 \\
\hline & 8.8 & BAtr & Dry aerosol & $9.55 \mathrm{E}+0-6.74 \mathrm{E}+4$ & PR & DW & $10-15$ & Marble & 100 & BBT & $\mathrm{S}+\mathrm{H}+\mathrm{V}$ & Plate, PF & 1 & 5 & 60 \\
\hline & 8.9 & BAtr + grime & Dry aerosol & $5.18 \mathrm{E}+2-5.03 \mathrm{E}+4$ & PR & DW & $89-90$ & Marble & 100 & BBT & $\mathrm{S}+\mathrm{H}+\mathrm{V}$ & Plate, PF & 1 & 5 & 59 \\
\hline & 8.10 & BAtr & Dry aerosol & $5.18 \mathrm{E}+2-5.03 \mathrm{E}+4$ & PR & DW & $89-90$ & Marble & 100 & BBT & $\mathrm{S}+\mathrm{H}+\mathrm{V}$ & Plate, PF & 1 & 5 & 60 \\
\hline \multirow{4}{*}{$\begin{array}{l}\text { Quizon et } \\
\text { al. } 2007\end{array}$} & 9.1 & BAtr & Wet aerosol & NR & PR & PBST & NR & PWB & 900 & PBST & $\mathrm{C}+\mathrm{V}+\mathrm{S}$ & SP/FP, TSA & 1 & 4 & 4 \\
\hline & 9.2 & BAtr & Wet aerosol & NR & PR & PBST & NR & $\mathrm{SS}$ & 900 & PBST & $\mathrm{C}+\mathrm{V}+\mathrm{S}$ & SP/FP, TSA & 1 & 4 & 10 \\
\hline & 9.3 & BAtr & Wet aerosol & NR & PR & PBST & NR & Vinyl & 900 & PBST & $\mathrm{C}+\mathrm{V}+\mathrm{S}$ & SP/FP, TSA & 1 & 4 & 4 \\
\hline & 9.4 & BAtr & Wet aerosol & NR & PR & PBST & NR & Wood & 900 & PBST & $\mathrm{C}+\mathrm{V}+\mathrm{S}$ & SP/FP, TSA & 1 & 4 & 4 \\
\hline \multirow{30}{*}{$\begin{array}{l}\text { Krauter et } \\
\text { al. } 2011\end{array}$} & 10.1 & BAtr & Liquid drops & $0.00248^{(b)}$ & Sp. stick & NB & $30-45$ & SS & 645.16 & PBST & $\mathrm{St}+\mathrm{C}+\mathrm{V}+\mathrm{S}$ & (c) & 1 & 1 & 10 \\
\hline & 10.2 & BAtr & Liquid drops & $0.00677^{(\mathrm{b})}$ & Sp. stick & NB & $30-45$ & SS & 645.16 & PBST & $\mathrm{St}+\mathrm{C}+\mathrm{V}+\mathrm{S}$ & (c) & 1 & 1 & 10 \\
\hline & 10.3 & BAtr & Liquid drops & $0.01669^{(b)}$ & Sp. stick & NB & $30-45$ & SS & 645.16 & PBST & $\mathrm{St}+\mathrm{C}+\mathrm{V}+\mathrm{S}$ & (c) & 1 & 1 & 10 \\
\hline & 10.4 & BAtr & Liquid drops & $0.01519^{(b)}$ & Sp. stick & NB & $30-45$ & SS & 645.16 & PBST & $\mathrm{St}+\mathrm{C}+\mathrm{V}+\mathrm{S}$ & (c) & 1 & 1 & 10 \\
\hline & 10.5 & BAtr & Liquid drops & $0.02253^{(\mathrm{b})}$ & Sp. stick & NB & $30-45$ & SS & 645.16 & PBST & $\mathrm{St}+\mathrm{C}+\mathrm{V}+\mathrm{S}$ & (c) & 1 & 1 & 10 \\
\hline & 10.6 & BAtr & Liquid drops & $0.03064^{(b)}$ & Sp. stick & NB & $30-45$ & SS & 645.16 & PBST & $\mathrm{St}+\mathrm{C}+\mathrm{V}+\mathrm{S}$ & (c) & 1 & 1 & 10 \\
\hline & 10.7 & BAtr & Liquid drops & $0.03725^{(\mathrm{b})}$ & Sp. stick & NB & $30-45$ & SS & 645.16 & PBST & $\mathrm{St}+\mathrm{C}+\mathrm{V}+\mathrm{S}$ & (c) & 1 & 1 & 10 \\
\hline & 10.8 & BAtr & Liquid drops & $0.15629^{(b)}$ & Sp. stick & NB & $30-45$ & SS & 645.16 & PBST & $\mathrm{St}+\mathrm{C}+\mathrm{V}+\mathrm{S}$ & (c) & 1 & 1 & 10 \\
\hline & 10.9 & BAtr & Liquid drops & $1.85380^{(b)}$ & Sp. stick & NB & $30-45$ & SS & 645.16 & PBST & $\mathrm{St}+\mathrm{C}+\mathrm{V}+\mathrm{S}$ & (c) & 1 & 1 & 10 \\
\hline & 10.10 & BAtr & Liquid drops & $0.00248^{(b)}$ & Sp. stick & NB & $30-45$ & CerT & 645.16 & PBST & $\mathrm{St}+\mathrm{C}+\mathrm{V}+\mathrm{S}$ & (c) & 1 & 1 & 10 \\
\hline & 10.11 & BAtr & Liquid drops & $0.00677^{(\mathrm{b})}$ & Sp. stick & NB & $30-45$ & CerT & 645.16 & PBST & $\mathrm{St}+\mathrm{C}+\mathrm{V}+\mathrm{S}$ & (c) & 1 & 1 & 10 \\
\hline & 10.12 & BAtr & Liquid drops & $0.01669^{(b)}$ & Sp. stick & NB & $30-45$ & CerT & 645.16 & PBST & $\mathrm{St}+\mathrm{C}+\mathrm{V}+\mathrm{S}$ & (c) & 1 & 1 & 10 \\
\hline & 10.13 & BAtr & Liquid drops & $0.01519^{(\mathrm{b})}$ & Sp. stick & NB & $30-45$ & CerT & 645.16 & PBST & $\mathrm{St}+\mathrm{C}+\mathrm{V}+\mathrm{S}$ & (c) & 1 & 1 & 10 \\
\hline & 10.14 & BAtr & Liquid drops & $0.02253^{(b)}$ & Sp. stick & NB & $30-45$ & CerT & 645.16 & PBST & $\mathrm{St}+\mathrm{C}+\mathrm{V}+\mathrm{S}$ & (c) & 1 & 1 & 10 \\
\hline & 10.15 & BAtr & Liquid drops & $0.03064^{(b)}$ & Sp. stick & NB & $30-45$ & CerT & 645.16 & PBST & $\mathrm{St}+\mathrm{C}+\mathrm{V}+\mathrm{S}$ & (c) & 1 & 1 & 10 \\
\hline & 10.16 & BAtr & Liquid drops & $0.03275^{(b)}$ & Sp. stick & NB & $30-45$ & CerT & 645.16 & PBST & $\mathrm{St}+\mathrm{C}+\mathrm{V}+\mathrm{S}$ & (c) & 1 & 1 & 9 \\
\hline & 10.17 & BAtr & Liquid drops & $0.15629^{(b)}$ & Sp. stick & NB & $30-45$ & CerT & 645.16 & PBST & $\mathrm{St}+\mathrm{C}+\mathrm{V}+\mathrm{S}$ & (c) & 1 & 1 & 10 \\
\hline & 10.18 & BAtr & Liquid drops & $1.85380^{(b)}$ & Sp. stick & NB & $30-45$ & CerT & 645.16 & PBST & $\mathrm{St}+\mathrm{C}+\mathrm{V}+\mathrm{S}$ & (c) & 1 & 1 & 10 \\
\hline & 10.19 & BAtr & Liquid drops & $0.00248^{(\mathrm{b})}$ & Sp. stick & NB & $30-45$ & Vinyl tile & 645.16 & PBST & $\mathrm{St}+\mathrm{C}+\mathrm{V}+\mathrm{S}$ & (c) & 1 & 1 & 10 \\
\hline & 10.20 & BAtr & Liquid drops & $0.00677^{(b)}$ & Sp. stick & NB & $30-45$ & Vinyl tile & 645.16 & PBST & $\mathrm{St}+\mathrm{C}+\mathrm{V}+\mathrm{S}$ & (c) & 1 & 1 & 10 \\
\hline & 10.21 & BAtr & Liquid drops & $0.01669^{(b)}$ & Sp. stick & NB & $30-45$ & Vinyl tile & 645.16 & PBST & $\mathrm{St}+\mathrm{C}+\mathrm{V}+\mathrm{S}$ & (c) & 1 & 1 & 10 \\
\hline & 10.22 & BAtr & Liquid drops & $0.01519^{(b)}$ & Sp. stick & NB & $30-45$ & Vinyl tile & 645.16 & PBST & $\mathrm{St}+\mathrm{C}+\mathrm{V}+\mathrm{S}$ & (c) & 1 & 1 & 10 \\
\hline & 10.23 & BAtr & Liquid drops & $0.02253^{(b)}$ & Sp. stick & NB & $30-45$ & Vinyl tile & 645.16 & PBST & $\mathrm{St}+\mathrm{C}+\mathrm{V}+\mathrm{S}$ & (c) & 1 & 1 & 10 \\
\hline & 10.24 & BAtr & Liquid drops & $0.03064^{(b)}$ & Sp. stick & NB & $30-45$ & Vinyl tile & 645.16 & PBST & $\mathrm{St}+\mathrm{C}+\mathrm{V}+\mathrm{S}$ & (c) & 1 & 1 & 9 \\
\hline & 10.25 & BAtr & Liquid drops & $0.03275^{(\mathrm{b})}$ & Sp. stick & NB & $30-45$ & Vinyl tile & 645.16 & PBST & $\mathrm{St}+\mathrm{C}+\mathrm{V}+\mathrm{S}$ & (c) & 1 & 1 & 10 \\
\hline & 10.26 & BAtr & Liquid drops & $0.15629^{(b)}$ & Sp. stick & NB & $30-45$ & Vinyl tile & 645.16 & PBST & $\mathrm{St}+\mathrm{C}+\mathrm{V}+\mathrm{S}$ & (c) & 1 & 1 & 9 \\
\hline & 10.27 & BAtr & Liquid drops & $1.85380^{(b)}$ & Sp. stick & NB & $30-45$ & Vinyl tile & 645.16 & PBST & $\mathrm{St}+\mathrm{C}+\mathrm{V}+\mathrm{S}$ & (c) & 1 & 1 & 10 \\
\hline & 10.28 & BAtr & Liquid drops & $0.00775^{(b)}$ & Sp. stick & NB & $30-45$ & FL & 645.16 & PBST & $\mathrm{St}+\mathrm{C}+\mathrm{V}+\mathrm{S}$ & (c) & 1 & 1 & 10 \\
\hline & 10.29 & BAtr & Liquid drops & $0.01535^{(b)}$ & Sp. stick & NB & $30-45$ & $\mathrm{FF}$ & 645.16 & PBST & $\mathrm{St}+\mathrm{C}+\mathrm{V}+\mathrm{S}$ & (c) & 1 & 1 & 10 \\
\hline & 10.30 & BAtr & Liquid drops & $0.02356^{(\mathrm{b})}$ & Sp. stick & NB & $30-45$ & FL & 645.16 & PBST & $\mathrm{St}+\mathrm{C}+\mathrm{V}+\mathrm{S}$ & (c) & 1 & 1 & 10 \\
\hline
\end{tabular}


Table A.2a Expanded summary of test conditions for wipe sampling. Acronyms and abbreviations are defined in Table 6. (cont.)

\begin{tabular}{|c|c|c|c|c|c|c|c|c|c|c|c|c|c|c|c|}
\hline Reference & $\begin{array}{c}\text { Test } \\
\# \\
\end{array}$ & Agent & $\begin{array}{l}\text { Agent } \\
\text { deposition }\end{array}$ & $\begin{array}{c}\text { Agent } \\
\text { concentration } \\
\left(\mathrm{CFU} / \mathrm{cm}^{2}\right)\end{array}$ & Wipe type & $\begin{array}{c}\text { Wetting } \\
\text { agent }\end{array}$ & $\begin{array}{c}\text { Relative } \\
\text { humidity } \\
(\%)\end{array}$ & $\begin{array}{c}\text { Surface } \\
\text { type }\end{array}$ & $\begin{array}{l}\text { Surface } \\
\text { area } \\
\text { sampled } \\
\left(\mathrm{cm}^{2}\right) \\
\end{array}$ & $\begin{array}{c}\text { Extraction } \\
\text { liquid }\end{array}$ & $\begin{array}{l}\text { Extraction } \\
\text { method }\end{array}$ & $\begin{array}{l}\text { Culture } \\
\text { method, } \\
\text { medium }\end{array}$ & $\begin{array}{c}\# \\
\text { labs } \\
\end{array}$ & $\begin{array}{l}\# \text { test } \\
\text { runs }\end{array}$ & $\begin{array}{c}\text { Total } \\
\text { \# test } \\
\text { samples } \\
\end{array}$ \\
\hline \multirow{24}{*}{$\begin{array}{l}\text { Krauter et } \\
\text { al. } 2011 \\
\text { (cont.) }\end{array}$} & 10.31 & BAtr & Liquid drops & $0.02289^{(\mathrm{b})}$ & Sp. stick & NB & $30-45$ & FL & 645.16 & PBST & $\mathrm{St}+\mathrm{C}+\mathrm{V}+\mathrm{S}$ & (c) & 1 & 1 & 10 \\
\hline & 10.32 & BAtr & Liquid drops & $0.03276^{(b)}$ & Sp. stick & NB & $30-45$ & FL & 645.16 & PBST & $\mathrm{St}+\mathrm{C}+\mathrm{V}+\mathrm{S}$ & (c) & 1 & 1 & 10 \\
\hline & 10.33 & BAtr & Liquid drops & $0.03834^{(b)}$ & Sp. stick & NB & $30-45$ & FL & 645.16 & PBST & $\mathrm{St}+\mathrm{C}+\mathrm{V}+\mathrm{S}$ & (c) & 1 & 1 & 10 \\
\hline & 10.34 & BAtr & Liquid drops & $0.05430^{(\mathrm{b})}$ & Sp. stick & NB & $30-45$ & FL & 645.16 & PBST & $\mathrm{St}+\mathrm{C}+\mathrm{V}+\mathrm{S}$ & (c) & 1 & 1 & 10 \\
\hline & 10.35 & BAtr & Liquid drops & $0.07905^{(\mathrm{b})}$ & Sp. stick & NB & $30-45$ & FL & 645.16 & PBST & $\mathrm{St}+\mathrm{C}+\mathrm{V}+\mathrm{S}$ & (c) & 1 & 1 & 10 \\
\hline & 10.36 & BAtr & Liquid drops & $0.15371^{(b)}$ & Sp. stick & NB & $30-45$ & $\mathrm{FF}$ & 645.16 & PBST & $\mathrm{St}+\mathrm{C}+\mathrm{V}+\mathrm{S}$ & (c) & 1 & 1 & 10 \\
\hline & 10.37 & BAtr & Liquid drops & $0.00775^{(b)}$ & Sp. stick & NB & $30-45$ & PW & 645.16 & PBST & $\mathrm{St}+\mathrm{C}+\mathrm{V}+\mathrm{S}$ & (c) & 1 & 1 & 10 \\
\hline & 10.38 & BAtr & Liquid drops & $0.01535^{(\mathrm{b})}$ & Sp. stick & NB & $30-45$ & PW & 645.16 & PBST & $\mathrm{St}+\mathrm{C}+\mathrm{V}+\mathrm{S}$ & (c) & 1 & 1 & 10 \\
\hline & 10.39 & BAtr & Liquid drops & $0.02356^{(b)}$ & Sp. stick & NB & $30-45$ & PW & 645.16 & PBST & $\mathrm{St}+\mathrm{C}+\mathrm{V}+\mathrm{S}$ & (c) & 1 & 1 & 10 \\
\hline & 10.40 & BAtr & Liquid drops & $0.02289^{(b)}$ & Sp. stick & NB & $30-45$ & PW & 645.16 & PBST & $\mathrm{St}+\mathrm{C}+\mathrm{V}+\mathrm{S}$ & (c) & 1 & 1 & 10 \\
\hline & 10.41 & BAtr & Liquid drops & $0.03276^{(b)}$ & Sp. stick & NB & $30-45$ & PW & 645.16 & PBST & $\mathrm{St}+\mathrm{C}+\mathrm{V}+\mathrm{S}$ & (c) & 1 & 1 & 10 \\
\hline & 10.42 & BAtr & Liquid drops & $0.03834^{(b)}$ & Sp. stick & NB & $30-45$ & PW & 645.16 & PBST & $\mathrm{St}+\mathrm{C}+\mathrm{V}+\mathrm{S}$ & (c) & 1 & 1 & 10 \\
\hline & 10.43 & BAtr & Liquid drops & $0.05430^{(b)}$ & Sp. stick & NB & $30-45$ & PW & 645.16 & PBST & $\mathrm{St}+\mathrm{C}+\mathrm{V}+\mathrm{S}$ & (c) & 1 & 1 & 10 \\
\hline & 10.44 & BAtr & Liquid drops & $0.07905^{(b)}$ & Sp. stick & NB & $30-45$ & PW & 645.16 & PBST & $\mathrm{St}+\mathrm{C}+\mathrm{V}+\mathrm{S}$ & (c) & 1 & 1 & 10 \\
\hline & 10.45 & BAtr & Liquid drops & $0.15371^{(b)}$ & Sp. stick & NB & $30-45$ & PW & 645.16 & PBST & $\mathrm{St}+\mathrm{C}+\mathrm{V}+\mathrm{S}$ & (c) & 1 & 1 & 10 \\
\hline & 10.46 & BAtr & Liquid drops & $0.00775^{(\mathrm{b})}$ & Sp. stick & NB & $30-45$ & PLCP & 645.16 & PBST & $\mathrm{St}+\mathrm{C}+\mathrm{V}+\mathrm{S}$ & (c) & 1 & 1 & 10 \\
\hline & 10.47 & BAtr & Liquid drops & $0.01535^{(b)}$ & Sp. stick & NB & $30-45$ & PLCP & 645.16 & PBST & $\mathrm{St}+\mathrm{C}+\mathrm{V}+\mathrm{S}$ & (c) & 1 & 1 & 10 \\
\hline & 10.48 & BAtr & Liquid drops & $0.02356^{(\mathrm{b})}$ & Sp. stick & NB & $30-45$ & PLCP & 645.16 & PBST & $\mathrm{St}+\mathrm{C}+\mathrm{V}+\mathrm{S}$ & (c) & 1 & 1 & 10 \\
\hline & 10.49 & BAtr & Liquid drops & $0.02289^{(b)}$ & Sp. stick & NB & $30-45$ & PLCP & 645.16 & PBST & $\mathrm{St}+\mathrm{C}+\mathrm{V}+\mathrm{S}$ & (c) & 1 & 1 & 10 \\
\hline & 10.50 & BAtr & Liquid drops & $0.03276^{(b)}$ & Sp. stick & NB & $30-45$ & PLCP & 645.16 & PBST & $\mathrm{St}+\mathrm{C}+\mathrm{V}+\mathrm{S}$ & (c) & 1 & 1 & 10 \\
\hline & 10.51 & BAtr & Liquid drops & $0.03834^{(\mathrm{b})}$ & Sp. stick & NB & $30-45$ & PLCP & 645.16 & PBST & $\mathrm{St}+\mathrm{C}+\mathrm{V}+\mathrm{S}$ & (c) & 1 & 1 & 10 \\
\hline & 10.52 & BAtr & Liquid drops & $0.05430^{(b)}$ & Sp. stick & NB & $30-45$ & PLCP & 645.16 & PBST & $\mathrm{St}+\mathrm{C}+\mathrm{V}+\mathrm{S}$ & (c) & 1 & 1 & 10 \\
\hline & 10.53 & BAtr & Liquid drops & $0.07905^{(b)}$ & Sp. stick & NB & $30-45$ & PLCP & 645.16 & PBST & $\mathrm{St}+\mathrm{C}+\mathrm{V}+\mathrm{S}$ & (c) & 1 & 1 & 10 \\
\hline & 10.54 & BAtr & Liquid drops & $0.15371^{(\mathrm{b})}$ & Sp. stick & NB & $30-45$ & PLCP & 645.16 & PBST & $\mathrm{St}+\mathrm{C}+\mathrm{V}+\mathrm{S}$ & (c) & 1 & 1 & 10 \\
\hline \multirow{11}{*}{$\begin{array}{l}\text { Rose et } \\
\text { al. } 2011\end{array}$} & 11.1 & BA Sterne & Liquid spread & 0.01349 & Sp. stick, DI & $\mathrm{NB}+\mathrm{ATD}$ & $\mathrm{NR}$ & SS & 645.16 & PBST & $\mathrm{St}+\mathrm{C}$ & SP/FP, TSAB & 9 & 1 & 17 \\
\hline & 11.2 & BA Sterne & Liquid spread & 0.27699 & Sp. stick, DI & $\mathrm{NB}+\mathrm{ATD}$ & NR & SS & 645.16 & PBST & $\mathrm{St}+\mathrm{C}$ & SP/FP, TSAB & 9 & 1 & 18 \\
\hline & 11.3 & BA Sterne & Liquid spread & 17.123 & Sp. stick, DI & $\mathrm{NB}+\mathrm{ATD}$ & NR & SS & 645.16 & PBST & $\mathrm{St}+\mathrm{C}$ & SP/FP, TSAB & 9 & 1 & 16 \\
\hline & 11.4 & BA Sterne & Liquid spread & 0.04046 & Sp. stick & $\mathrm{NB}+\mathrm{ATD}$ & NR & SS & 645.16 & PBST & $\mathrm{St}+\mathrm{C}$ & SP/FP, TSAB & 9 & 1 & 63 \\
\hline & 11.5 & BA Sterne & Liquid spread & 0.83080 & Sp. stick & $\mathrm{NB}+\mathrm{ATD}$ & NR & SS & 645.16 & PBST & $\mathrm{St}+\mathrm{C}$ & SP/FP, TSAB & 9 & 1 & 63 \\
\hline & 11.6 & BA Sterne & Liquid spread & 51.367 & Sp. stick & $\mathrm{NB}+\mathrm{ATD}$ & NR & SS & 645.16 & PBST & $\mathrm{St}+\mathrm{C}$ & SP/FP, TSAB & 8 & 1 & 56 \\
\hline & 11.7 & BA Sterne & Liquid spread & 15.5 & Rayon gauze & PBST & NR & SS & 645.16 & PBST & $\mathrm{St}+\mathrm{C}$ & SP/FP, TSAB & 1 & 1 & 10 \\
\hline & 11.8 & BA Sterne & Liquid spread & 15.5 & Sponge wipe & DE broth & NR & SS & 645.16 & PBST & $\mathrm{St}+\mathrm{C}$ & SP/FP, TSAB & 1 & 1 & 10 \\
\hline & 11.9 & BA Sterne & Liquid spread & 15.5 & Sponge wipe & $\mathrm{BB}$ & NR & SS & 645.16 & PBST & $\mathrm{St}+\mathrm{C}$ & SP/FP, TSAB & 1 & 1 & 10 \\
\hline & 11.10 & BA Sterne & Liquid spread & 15.5 & Sponge stick & PBST & NR & SS & 645.16 & PBST & $\mathrm{St}+\mathrm{C}$ & SP/FP, TSAB & 1 & 1 & 10 \\
\hline & 11.11 & BA Sterne & Liquid spread & $\begin{array}{c}0.0155,0.155 \\
15.5\end{array}$ & PEF sponge & PBST & NR & SS & 645.16 & PBST & $\mathrm{St}+\mathrm{C}$ & SP/FP, TSAB & 1 & 3 & 15 \\
\hline
\end{tabular}


Table A.2a Expanded summary of test conditions for wipe sampling. Acronyms and abbreviations are defined in Table . (cont.).

\begin{tabular}{|c|c|c|c|c|c|c|c|c|c|c|c|c|c|c|c|}
\hline Reference & $\begin{array}{c}\text { Test } \\
\# \\
\end{array}$ & Agent & $\begin{array}{l}\text { Agent } \\
\text { deposition }\end{array}$ & $\begin{array}{c}\text { Agent } \\
\text { concentration } \\
\left(\mathrm{CFU} / \mathrm{cm}^{2}\right) \\
\end{array}$ & Wipe type & $\begin{array}{c}\text { Wetting } \\
\text { agent }\end{array}$ & $\begin{array}{c}\text { Wipe } \\
\text { technique }\end{array}$ & $\begin{array}{c}\text { Surface } \\
\text { type }\end{array}$ & $\begin{array}{c}\text { Surface } \\
\text { area } \\
\text { sampled } \\
\left(\mathrm{cm}^{2}\right) \\
\end{array}$ & $\begin{array}{c}\text { Extraction } \\
\text { liquid }\end{array}$ & $\begin{array}{c}\text { Extraction } \\
\text { method }\end{array}$ & $\begin{array}{l}\text { Culture } \\
\text { method, } \\
\text { medium }\end{array}$ & $\begin{array}{c}\# \\
\text { labs } \\
\end{array}$ & $\begin{array}{c}\# \text { test } \\
\text { runs }\end{array}$ & $\begin{array}{c}\text { Total } \\
\text { \# test } \\
\text { samples } \\
\end{array}$ \\
\hline \multirow{4}{*}{$\begin{array}{l}\text { Montgomery } \\
\text { and Camp } \\
2008\end{array}$} & 12.1 & BA Sterne & EWD & NR & PR gauze & PBSTr & Rough & HVAC filter & 100 & PBSTr & Shake+C & Plate, NR & 1 & 3 & 5 \\
\hline & 12.2 & BA Sterne & EWD & NR & PR gauze & PBSTr & Smooth & HVAC filter & 100 & PBSTr & Shake $+\mathrm{C}$ & Plate, NR & 1 & 2 & 2 \\
\hline & 12.3 & BA Sterne & EWD & NR & PR pad & PBSTr & Rough & HVAC filter & 100 & PBSTr & Shake $+C$ & Plate, NR & 1 & 2 & 4 \\
\hline & 12.4 & BA Sterne & EWD & NR & PR pad & PBSTr & Smooth & HVAC filter & 100 & PBSTr & Shake $+\mathrm{C}$ & Plate, NR & 1 & 2 & 4 \\
\hline
\end{tabular}

(a) $1 \mathrm{E}+2$ to $1 \mathrm{E}+5 /$ sample, sample surface area not reported.

(b) Mean of 230 positive control samples paired with 30 test coupons in a given test run. Krauter et al. (2011) also report the \%RSDs of these values.

(c) Plate with growth medium, or filter plate with TSA. 
Table A.2b Expanded summary of test results for wipe sampling. Acronyms and abbreviations are defined in Table 6 .

\begin{tabular}{|c|c|c|c|c|c|c|c|c|c|c|c|c|c|c|c|c|c|}
\hline \multirow[b]{2}{*}{ Reference } & \multirow[b]{2}{*}{$\begin{array}{c}\text { Test } \\
\#\end{array}$} & \multicolumn{5}{|c|}{$\begin{array}{c}\text { Recovery concentration results - } \\
\text { Mean \& \%RSDs }\end{array}$} & \multicolumn{5}{|c|}{$\begin{array}{c}\text { Recovery efficiency (RE) - } \\
\text { Mean \& \%RSDs }\end{array}$} & \multicolumn{6}{|c|}{ LOD, FNR, and FPR } \\
\hline & & $\begin{array}{c}\text { Mean } \\
\left(\mathrm{CFU} / \mathrm{cm}^{2}\right)\end{array}$ & $\begin{array}{l}\text { Lab } \\
\% \text { RSD }\end{array}$ & $\begin{array}{l}\text { Run } \\
\% \text { RSD }\end{array}$ & $\begin{array}{c}\text { Sample- } \\
\text { within-run } \\
\text { \%RSD }\end{array}$ & $\begin{array}{c}\text { Total } \\
\% \text { RSD }\end{array}$ & \begin{tabular}{|c|}
$\mathrm{RE}$ \\
mean \\
$(\%)$
\end{tabular} & $\begin{array}{l}\text { RE lab } \\
\text { \%RSD }\end{array}$ & $\begin{array}{l}\text { RE run } \\
\% \text { RSD }\end{array}$ & $\begin{array}{l}\text { RE sample- } \\
\text { within-run } \\
\% \mathrm{RSD}\end{array}$ & \begin{tabular}{|c|}
$\mathrm{RE}$ \\
total \\
$\% \mathrm{RSD}$
\end{tabular} & $\begin{array}{l}\text { Positive } \\
\text { result } \\
\text { (CFU) }\end{array}$ & $\begin{array}{l}\text { LOD } \\
\text { defin- } \\
\text { ition }\end{array}$ & $\begin{array}{c}\text { LOD } \\
\left(\mathrm{CFU} / \mathrm{cm}^{2}\right)\end{array}$ & $\begin{array}{c}\text { LOD SD } \\
\text { or } 95 \% \mathrm{CI} \\
\left(\mathrm{CFU} / \mathrm{cm}^{2}\right)\end{array}$ & FNR & FPR \\
\hline \multirow{6}{*}{$\begin{array}{l}\text { Estill et } \\
\text { al. } 2009\end{array}$} & 1.1 & 0.023 & 9 & 0 & 200 & 200 & 31 & 14 & 0 & 160 & 160 & & & & & & \\
\hline & 1.2 & 0.084 & 27 & 28 & 76 & 85 & 22 & 29 & 13 & 78 & 84 & $\geq 3$ & $\mathrm{LOD}_{95}$ & 0.15 & $\begin{array}{c}95 \% \mathrm{Cl}: \\
0076-0.84\end{array}$ & NR & $\begin{array}{r}4 / 30= \\
0133\end{array}$ \\
\hline & 1.3 & 0.500 & 25 & 45 & 61 & 80 & 18 & 19 & 8 & 53 & 57 & & & & & & \\
\hline & 1.4 & 0.017 & 26 & 0 & 180 & 182 & 120 & 0 & 0 & 220 & 220 & & & & & & \\
\hline & 1.5 & 0.034 & 9 & 52 & 100 & 113 & 21 & 11 & 58 & 99 & 120 & $\geq 1$ & $\mathrm{LOD}_{95}$ & 0.099 & 95\% Cl: & NR & $0 / 30=$ \\
\hline & 1.6 & 0.350 & 9 & 47 & 37 & 60 & 23 & 8 & 63 & 40 & 75 & & & & & & \\
\hline \multirow{6}{*}{$\begin{array}{l}\text { Brown et } \\
\text { al. } 2007 \mathrm{a}\end{array}$} & 2.1 & NR & NA & NR & NR & NA & 31.2 & NA & NR & 32 & NA & $\geq 1 \mathrm{CFU} / \mathrm{ml}$ & NR & 3.6 & NR & NR & NR \\
\hline & 2.2 & NR & NA & NR & NR & NA & 39.2 & NA & NR & 35 & NA & $\geq 1 \mathrm{CFU} / \mathrm{ml}$ & NR & 3.6 & NR & NR & NR \\
\hline & 2.3 & NR & NA & NR & NR & NA & 32.5 & NA & NR & 48 & NA & $\geq 1 \mathrm{CFU} / \mathrm{ml}$ & NR & 4.2 & NR & NR & NR \\
\hline & 2.4 & NR & NA & NR & NR & NA & 25.2 & NA & NR & 59 & NA & $\geq 1 \mathrm{CFU} / \mathrm{ml}$ & NR & 4.2 & NR & NR & NR \\
\hline & 2.5 & NR & NA & NR & NR & NA & 93.2 & NA & NR & 9 & NA & $\geq 1 \mathrm{CFU} / \mathrm{ml}$ & NR & NR & NR & NR & NR \\
\hline & 2.6 & NR & NA & NR & NR & NA & 99.9 & NA & NR & 0.1 & NA & $\geq 1 \mathrm{CFU} / \mathrm{ml}$ & NR & NR & NR & NR & NR \\
\hline \multirow{13}{*}{$\begin{array}{l}\text { Buttner et } \\
\text { al. } 2001\end{array}$} & 3.1 & NR & NA & NR & NR & NA & 74.3 & NA & NR & 7 & NA & NR & NR & NR & NR & NR & NR \\
\hline & 3.2 & 1283 & NA & $14^{(\mathrm{a})}$ & (a) & NA & NR & NA & NR & NR & NA & NR & NR & NR & NR & NR & NR \\
\hline & 3.3 & 268 & NA & $17^{(\mathrm{a})}$ & (a) & NA & NR & NA & NR & NR & NA & NR & NR & NR & NR & NR & NR \\
\hline & 3.4 & 135 & NA & $10^{(\mathrm{a})}$ & (a) & NA & NR & NA & NR & NR & NA & NR & NR & NR & NR & NR & NR \\
\hline & 3.5 & 273 & NA & $51^{\text {(a) }}$ & (a) & NA & NR & NA & NR & NR & NA & NR & NR & NR & NR & NR & NR \\
\hline & 3.6 & 983 & NA & $33^{\text {(a) }}$ & (a) & NA & NR & NA & NR & NR & NA & NR & NR & NR & NR & NR & NR \\
\hline & 3.7 & 512 & NA & $34^{(\mathrm{a})}$ & (a) & NA & NR & NA & NR & NR & NA & NR & NR & NR & NR & NR & NR \\
\hline & 3.8 & 127 & NA & $42^{(a)}$ & (a) & NA & NR & NA & NR & NR & NA & NR & NR & NR & NR & NR & NR \\
\hline & 3.9 & 326 & NA & $27^{(\mathrm{a})}$ & (a) & NA & NR & NA & NR & NR & NA & NR & NR & NR & NR & NR & NR \\
\hline & 3.10 & 817 & NA & $101^{(a)}$ & (a) & NA & NR & NA & NR & NR & NA & NR & NR & NR & NR & NR & NR \\
\hline & 3.11 & 234 & NA & $0.6^{(\mathrm{a})}$ & (a) & NA & NR & NA & NR & NR & NA & NR & NR & NR & NR & NR & NR \\
\hline & 3.12 & 155 & NA & $40^{(\mathrm{a})}$ & (a) & NA & NR & NA & NR & NR & NA & NR & NR & NR & NR & NR & NR \\
\hline & 3.13 & 339 & NA & $36^{(\mathrm{a})}$ & (a) & NA & NR & NA & NR & NR & NA & NR & NR & NR & NR & NR & NR \\
\hline \multirow{12}{*}{$\begin{array}{l}\text { Valentine } \\
\text { et al. } 2008\end{array}$} & 4.1 & NR & NA & NR & NR & NA & 5.4 & NA & NR & 32 & NA & NR & NR & NR & NR & NR & NR \\
\hline & 4.2 & NR & NA & NR & NR & NA & 7.1 & NA & NR & 18 & NA & NR & NR & NR & NR & NR & NR \\
\hline & 4.3 & NR & NA & NR & NR & NA & 7.9 & NA & NR & 30 & NA & NR & NR & NR & NR & NR & NR \\
\hline & 4.4 & NR & NA & NR & NR & NA & 5.0 & NA & NR & 34 & NA & NR & NR & NR & NR & NR & NR \\
\hline & 4.5 & NR & NA & NR & NR & NA & 6.0 & NA & NR & 33 & NA & NR & NR & NR & NR & NR & NR \\
\hline & 4.6 & NR & NA & NR & NR & NA & 5.3 & NA & NR & 22 & NA & NR & NR & NR & NR & NR & NR \\
\hline & 4.7 & NR & NA & NR & NR & NA & 4.1 & NA & NR & 84 & NA & NR & NR & NR & NR & NR & NR \\
\hline & 4.8 & NR & NA & NR & NR & NA & 5.8 & NA & NR & 40 & NA & NR & NR & NR & NR & NR & NR \\
\hline & 4.9 & NR & NA & NR & NR & NA & 5.9 & NA & NR & 62 & NA & NR & NR & NR & NR & NR & NR \\
\hline & 4.10 & NR & NA & NR & NR & NA & 1.4 & NA & NR & 20 & NA & NR & NR & NR & NR & NR & NR \\
\hline & 4.11 & NR & NA & NR & NR & NA & 2.1 & NA & NR & 20 & NA & NR & NR & NR & NR & NR & NR \\
\hline & 4.12 & NR & NA & NR & NR & NA & 2.0 & NA & NR & 28 & NA & NR & NR & NR & NR & NR & NR \\
\hline
\end{tabular}


Table A.2b Expanded summary of test results for wipe sampling. Acronyms and abbreviations are defined in Table 6. (cont.)

\begin{tabular}{|c|c|c|c|c|c|c|c|c|c|c|c|c|c|c|c|c|c|}
\hline \multirow[b]{2}{*}{ Reference } & \multirow[b]{2}{*}{$\begin{array}{c}\text { Test } \\
\#\end{array}$} & \multicolumn{5}{|c|}{$\begin{array}{c}\text { Recovery concentration results - } \\
\text { Mean \& \%RSDs }\end{array}$} & \multicolumn{5}{|c|}{$\begin{array}{l}\text { Recovery efficiency }(\mathrm{RE})- \\
\text { Mean \& \%RSDs }\end{array}$} & \multicolumn{6}{|c|}{ LOD, FNR, and FPR } \\
\hline & & $\begin{array}{c}\text { Mean } \\
\left(\mathrm{CFU} / \mathrm{cm}^{2}\right)\end{array}$ & $\begin{array}{l}\text { Lab } \\
\% \text { RSD }\end{array}$ & $\begin{array}{l}\text { Run } \\
\% \text { RSD }\end{array}$ & $\begin{array}{c}\text { Sample- } \\
\text { within-run } \\
\% \text { RSD }\end{array}$ & $\begin{array}{c}\text { Total } \\
\% \text { RSD }\end{array}$ & $\begin{array}{c}\mathrm{RE} \\
\text { mean } \\
(\%)\end{array}$ & $\begin{array}{l}\text { RE lab } \\
\% \text { RSD }\end{array}$ & $\begin{array}{l}\text { RE run } \\
\% \text { RSD }\end{array}$ & $\begin{array}{c}\text { RE sample- } \\
\text { within-run } \\
\% \text { RSD }\end{array}$ & \begin{tabular}{|c|}
$\mathrm{RE}$ \\
total \\
$\% \mathrm{RSD}$ \\
\end{tabular} & $\begin{array}{c}\text { Positive } \\
\text { result } \\
(\mathrm{CFU})\end{array}$ & $\begin{array}{l}\text { LOD } \\
\text { defin- } \\
\text { ition }\end{array}$ & $\begin{array}{c}\mathrm{LOD} \\
\left(\mathrm{CFU} / \mathrm{cm}^{2}\right)\end{array}$ & $\begin{array}{c}\text { LOD SD } \\
\text { or } 95 \% \mathrm{CI} \\
\left(\mathrm{CFU} / \mathrm{cm}^{2}\right)\end{array}$ & FNR & FPR \\
\hline \multirow{3}{*}{$\begin{array}{l}\text { Valentine } \\
\text { et al. } 2008 \\
\text { (cont.) }\end{array}$} & 4.13 & NR & NA & NR & NR & NA & 2.6 & NA & NR & 38 & NA & NR & NR & $\mathrm{NR}$ & $\mathrm{NR}$ & NR & NR \\
\hline & 4.14 & NR & NA & NR & NR & NA & 3.6 & NA & NR & 37 & NA & NR & NR & NR & NR & NR & NR \\
\hline & 4.15 & NR & NA & NR & NR & NA & 4.2 & NA & NR & 22 & NA & NR & NR & NR & NR & NR & NR \\
\hline \multirow{18}{*}{$\begin{array}{l}\text { Buttner et } \\
\text { al. } 2004 \mathrm{a}\end{array}$} & 5.1 & 1786 & NA & $36^{(\mathrm{a})}$ & (a) & NA & NR & NA & NR & NR & NA & NR & NR & NR & NR & NR & NR \\
\hline & 5.2 & 938 & NA & $8^{(\mathrm{a})}$ & (a) & NA & NR & NA & NR & NR & NA & NR & NR & NR & NR & NR & NR \\
\hline & 5.3 & 1452 & NA & $36^{(\mathrm{a})}$ & (a) & NA & NR & NA & NR & NR & NA & NR & NR & NR & NR & NR & NR \\
\hline & 5.4 & 1592 & NA & $0^{(\mathrm{a})}$ & (a) & NA & NR & NA & NR & NR & NA & NR & NR & NR & NR & NR & NR \\
\hline & 5.5 & 1387 & NA & $16^{(\mathrm{a})}$ & (a) & NA & NR & NA & NR & NR & NA & NR & NR & NR & NR & NR & NR \\
\hline & 5.6 & 1592 & NA & $16^{(\mathrm{a})}$ & (a) & NA & NR & NA & NR & NR & NA & NR & NR & NR & NR & NR & NR \\
\hline & 5.7 & 1076 & NA & $36^{(\mathrm{a})}$ & (a) & NA & NR & NA & NR & NR & NA & NR & NR & NR & NR & NR & NR \\
\hline & 5.8 & 1236 & NA & $36^{(\mathrm{a})}$ & (a) & NA & NR & NA & NR & NR & NA & NR & NR & NR & NR & NR & NR \\
\hline & 5.9 & 817 & NA & $36^{(\mathrm{a})}$ & (a) & NA & NR & NA & NR & NR & NA & NR & NR & NR & NR & NR & NR \\
\hline & 5.10 & 711 & NA & $24^{(\mathrm{a})}$ & (a) & NA & NR & NA & NR & NR & NA & NR & NR & NR & NR & NR & NR \\
\hline & 5.11 & 1180 & NA & $24^{(\mathrm{a})}$ & (a) & NA & NR & NA & NR & NR & NA & NR & NR & NR & NR & NR & NR \\
\hline & 5.12 & 6950 & NA & $44^{(\mathrm{a})}$ & (a) & NA & NR & NA & NR & NR & NA & NR & NR & NR & NR & NR & NR \\
\hline & 5.13 & 820 & NA & NA & 3 & NA & NR & NA & NR & NR & NA & NR & NR & NR & NR & NR & NR \\
\hline & 5.14 & 506 & NA & NA & 95 & NA & NR & NA & NR & NR & NA & NR & NR & NR & NR & NR & NR \\
\hline & 5.15 & 841 & NA & NA & NA & NA & NR & NA & NR & NR & NA & NR & NR & NR & NR & NR & NR \\
\hline & 5.16 & 637 & NA & NA & 12 & NA & NR & NA & NR & NR & NA & NR & NR & NR & NR & NR & NR \\
\hline & 5.17 & 883 & NA & NA & 4 & NA & NR & NA & NR & NR & NA & NR & NR & NR & NR & NR & NR \\
\hline & 5.18 & 667 & NA & NA & 13 & NA & NR & NA & NR & NR & NA & NR & NR & NR & NR & NR & NR \\
\hline \multirow{10}{*}{$\begin{array}{l}\text { Buttner et } \\
\text { al. } 2004 b\end{array}$} & 6.1 & 3.35 & NA & NR & NR & NA & 18.4 & NA & NR & NR & NA & $\geq 1 \mathrm{CFU} / \mathrm{ml}$ & NR & 0.0042 & $\mathrm{SE}=5.8 \mathrm{E}-04$ & NR & NR \\
\hline & 6.2 & 2.05 & NA & NR & NR & NA & 11.3 & NA & NR & NR & NA & $\geq 1 \mathrm{CFU} / \mathrm{ml}$ & NR & 0.01005 & $\mathrm{SE}=1.02 \mathrm{E}-03$ & NR & NR \\
\hline & 6.3 & NR & NA & NR & NR & NA & NR & NA & NR & NR & NA & $\geq 1 \mathrm{CFU} / \mathrm{ml}$ & NR & 0.0042 & $\mathrm{SE}=5.8 \mathrm{E}-04$ & NR & NR \\
\hline & 6.4 & NR & NA & NR & NR & NA & NR & NA & NR & NR & NA & $\geq 1 \mathrm{CFU} / \mathrm{ml}$ & NR & 0.01005 & $\mathrm{SE}=1.02 \mathrm{E}-03$ & NR & NR \\
\hline & 6.5 & 395 & NA & 27 & NR & NA & NR & NA & NR & NR & NA & NR & NR & NR & NR & NR & NR \\
\hline & 6.6 & 590 & NA & 14 & NR & NA & NR & NA & NR & NR & NA & NR & NR & NR & NR & NR & NR \\
\hline & 6.7 & 360 & NA & 38 & NR & NA & NR & NA & NR & NR & NA & NR & NR & NR & NR & NR & NR \\
\hline & 6.8 & 360 & NA & 39 & NR & NA & NR & NA & NR & NR & NA & NR & NR & NR & NR & NR & NR \\
\hline & 6.9 & 215 & NA & 23 & NR & NA & NR & NA & NR & NR & NA & NR & NR & NR & NR & NR & NR \\
\hline & 6.10 & 115 & NA & 84 & NR & NA & NR & NA & NR & NR & NA & NR & NR & NR & NR & NR & NR \\
\hline \multirow{6}{*}{$\begin{array}{l}\text { Frawley et } \\
\text { al. } 2008\end{array}$} & 7.1 & NR & NA & NR & NR & NA & 0.9 & NA & NR & 63 & NA & NR & NR & NR & NR & NR & NR \\
\hline & 7.2 & NR & NA & NR & NR & NA & 0.2 & NA & NR & 70 & NA & NR & NR & NR & NR & NR & NR \\
\hline & 7.3 & NR & NA & NR & NR & NA & 0.9 & NA & NR & 55 & NA & NR & NR & NR & NR & NR & NR \\
\hline & 7.4 & NR & NA & NR & NR & NA & 6.6 & NA & NR & 60 & NA & NR & NR & NR & NR & NR & NR \\
\hline & 7.5 & NR & NA & NR & NR & NA & 6.0 & NA & NR & 58 & NA & NR & NR & NR & NR & NR & NR \\
\hline & 7.6 & NR & NA & NR & NR & NA & 4.0 & NA & NR & 46 & NA & NR & NR & NR & NR & NR & NR \\
\hline
\end{tabular}


Table A.2b Expanded summary of test results for wipe sampling. Acronyms and abbreviations are defined in Table 6. (cont.)

\begin{tabular}{|c|c|c|c|c|c|c|c|c|c|c|c|c|c|c|c|c|c|}
\hline \multirow[b]{2}{*}{ Reference } & \multirow[b]{2}{*}{$\begin{array}{c}\text { Test } \\
\#\end{array}$} & \multicolumn{5}{|c|}{$\begin{array}{c}\text { Recovery concentration results - } \\
\text { Mean \& \%RSDs }\end{array}$} & \multicolumn{5}{|c|}{$\begin{array}{c}\text { Recovery efficiency (RE) - } \\
\text { Mean \& \%RSDs }\end{array}$} & \multicolumn{6}{|c|}{ LOD, FNR, and FPR } \\
\hline & & $\begin{array}{c}\text { Mean } \\
\left(\mathrm{CFU} / \mathrm{cm}^{2}\right)\end{array}$ & $\begin{array}{l}\text { Lab } \\
\% \text { RSD }\end{array}$ & $\begin{array}{c}\text { Run } \\
\% \text { RSD }\end{array}$ & $\begin{array}{c}\text { Sample- } \\
\text { within-run } \\
\% \text { RSD } \\
\end{array}$ & $\begin{array}{c}\text { Total } \\
\% \text { RSD }\end{array}$ & \begin{tabular}{|c|}
$\mathrm{RE}$ \\
mean \\
$(\%)$ \\
\end{tabular} & $\begin{array}{l}\text { RE lab } \\
\text { \%RSD }\end{array}$ & $\begin{array}{l}\text { RE run } \\
\% \text { RSD }\end{array}$ & $\begin{array}{c}\text { RE sample- } \\
\text { within-run } \\
\% \text { RSD } \\
\end{array}$ & \begin{tabular}{|c|}
$\mathrm{RE}$ \\
total \\
$\% \mathrm{RSD}$ \\
\end{tabular} & $\begin{array}{c}\text { Positive } \\
\text { result } \\
(\mathrm{CFU}) \\
\end{array}$ & $\begin{array}{c}\text { LOD } \\
\text { defin- } \\
\text { ition } \\
\end{array}$ & $\begin{array}{c}\text { LOD } \\
\left(\mathrm{CFU} / \mathrm{cm}^{2}\right)\end{array}$ & $\begin{array}{c}\text { LOD SD } \\
\text { or } 95 \% \mathrm{CI} \\
\left(\mathrm{CFU} / \mathrm{cm}^{2}\right)\end{array}$ & FNR & FPR \\
\hline \multirow{10}{*}{$\begin{array}{l}\text { Einfeld et } \\
\text { al. } 2011\end{array}$} & 8.1 & NR & NA & NR & NR & NA & 96.8 & NA & $29^{(\mathrm{b})}$ & (b) & NA & NR & NR & NR & NR & NR & NR \\
\hline & 8.2 & NR & NA & NR & NR & NA & 79.7 & NA & $43^{(\mathrm{b})}$ & (b) & NA & NR & NR & NR & NR & NR & NR \\
\hline & 8.3 & NR & NA & NR & NR & NA & 19.8 & NA & $67^{(\mathrm{b})}$ & (b) & NA & NR & NR & NR & NR & NR & NR \\
\hline & 8.4 & NR & NA & NR & NR & NA & 18.0 & NA & $81^{(\mathrm{b})}$ & (b) & NA & NR & NR & NR & NR & NR & NR \\
\hline & 8.5 & NR & NA & NR & NR & NA & 54.7 & NA & $78^{(\mathrm{b})}$ & (b) & NA & NR & NR & NR & NR & NR & NR \\
\hline & 8.6 & NR & NA & NR & NR & NA & 33.3 & NA & $81^{(\mathrm{b})}$ & (b) & NA & NR & NR & NR & NR & NR & NR \\
\hline & 8.7 & NR & NA & NR & NR & NA & 59.5 & NA & $73^{(\mathrm{b})}$ & (b) & NA & NR & NR & NR & NR & NR & NR \\
\hline & 8.8 & NR & NA & NR & NR & NA & 48.2 & NA & $71^{(\mathrm{b})}$ & (b) & NA & NR & NR & NR & NR & NR & NR \\
\hline & 8.9 & NR & NA & NR & NR & NA & 68.5 & NA & $96^{(\mathrm{b})}$ & (b) & NA & NR & NR & NR & NR & NR & NR \\
\hline & 8.10 & NR & NA & NR & NR & NA & 43.6 & NA & $73^{(\mathrm{b})}$ & (b) & NA & NR & NR & NR & NR & NR & NR \\
\hline \multirow{4}{*}{$\begin{array}{l}\text { Quizon et } \\
\text { al. } 2007\end{array}$} & 9.1 & 6670 & NA & $8^{(a)}$ & (a) & NA & NR & NA & $\mathrm{NR}$ & NR & NA & NR & NR & NR & NR & NR & NR \\
\hline & 9.2 & 16335 & NA & $20^{(\mathrm{a})}$ & (a) & NA & 39.5 & NA & (c) & (c) & NA & NR & NR & NR & NR & NR & NR \\
\hline & 9.3 & 12000 & NA & $11^{(\mathrm{a})}$ & (a) & NA & NR & NA & NR & NR & NA & NR & NR & NR & NR & NR & NR \\
\hline & 9.4 & 14615 & NA & $9^{(\mathrm{a})}$ & (a) & NA & NR & NA & NR & NR & NA & NR & NR & NR & NR & NR & NR \\
\hline \multirow{24}{*}{$\begin{array}{l}\text { Krauter et } \\
\text { al. } 2011\end{array}$} & 10.1 & 0.00155 & NA & NR & 105 & NA & 62.5 & NA & NR & 106 & NA & $\geq 1$ & & & & 0.600 & 0.0 \\
\hline & 10.2 & 0.00372 & NA & NR & 77 & NA & 55.0 & NA & NR & 77 & NA & $\geq 1$ & & & & 0.367 & 0.0 \\
\hline & 10.3 & 0.00961 & NA & NR & 32 & NA & 57.6 & NA & NR & 32 & NA & $\geq 1$ & & & & 0.033 & 0.0 \\
\hline & 10.4 & 0.00961 & NA & NR & 28 & NA & 63.3 & NA & NR & 28 & NA & $\geq 1$ & & & 95\% CI: & 0.0 & 0.0 \\
\hline & 10.5 & 0.01116 & NA & NR & 14 & NA & 49.5 & NA & NR & 14 & NA & $\geq 1$ & $\mathrm{LOD}_{95}$ & 0.013 & $(0.010,0.015)$ & 0.0 & 0.0 \\
\hline & 10.6 & 0.01581 & NA & NR & 20 & NA & 51.6 & NA & NR & 29 & NA & $\geq 1$ & $\mathrm{LOD}_{90}$ & 0.015 & NR & 0.0 & 0.0 \\
\hline & 10.7 & 0.01333 & NA & NR & 22 & NA & 35.8 & NA & NR & 22 & NA & $\geq 1$ & & & & 0.0 & 0.0 \\
\hline & 10.8 & 0.07194 & NA & NR & 14 & NA & 46.0 & NA & NR & 14 & NA & $\geq 1$ & & & & 0.0 & 0.0 \\
\hline & 10.9 & 0.97402 & NA & NR & 8.8 & NA & 52.5 & NA & NR & 8.9 & NA & $\geq 1$ & & & & 0.0 & 0.0 \\
\hline & 10.10 & 0.00093 & NA & NR & 161 & NA & 37.5 & NA & NR & 161 & NA & $\geq 1$ & & & & 0.800 & 0.0 \\
\hline & 10.11 & 0.00217 & NA & NR & 96 & NA & 32.1 & NA & NR & 96 & NA & $\geq 1$ & & & & 0.567 & 0.0 \\
\hline & 10.12 & 0.00899 & NA & NR & 60 & NA & 53.9 & NA & NR & 60 & NA & $\geq 1$ & & & & 0.100 & 0.0 \\
\hline & 10.13 & 0.01147 & NA & NR & 42 & NA & 75.5 & NA & NR & 42 & NA & $\geq 1$ & & & 95\% CI: & 0.0 & 0.0 \\
\hline & 10.14 & 0.01085 & NA & NR & 28 & NA & 48.2 & NA & NR & 28 & NA & $\geq 1$ & $\mathrm{LOD}_{95}$ & 0.013 & $(0.007,0.015)$ & 0.0 & 0.0 \\
\hline & 10.15 & 0.01581 & NA & NR & 28 & NA & 51.6 & NA & NR & 28 & NA & $\geq 1$ & $\mathrm{LOD}_{90}$ & 0.015 & $\mathrm{NR}$ & 0.033 & 0.0 \\
\hline & 10.16 & 0.01584 & NA & NR & 18 & NA & 42.5 & NA & NR & 18 & NA & $\geq 1$ & & & & 0.0 & 0.0 \\
\hline & 10.17 & 0.07037 & NA & NR & 12 & NA & 45.0 & NA & NR & 12 & NA & $\geq 1$ & & & & 0.0 & 0.0 \\
\hline & 10.18 & 0.99355 & NA & NR & 6.6 & NA & 53.6 & NA & NR & 6.6 & NA & $\geq 1$ & & & & 0.0 & 0.0 \\
\hline & 10.19 & 0.00031 & NA & NR & 316 & NA & 12.5 & NA & NR & 316 & NA & $\geq 1$ & & & & 0.933 & 0.0 \\
\hline & 10.10 & 0.00217 & NA & NR & 96 & NA & 32.1 & NA & NR & 96 & NA & $\geq 1$ & & & & 0.600 & 0.0 \\
\hline & 10.21 & 0.01085 & NA & NR & 43 & NA & 65.0 & NA & NR & 43 & NA & $\geq 1$ & & & & 0.0 & 0.0 \\
\hline & 10.22 & 0.00713 & NA & NR & 21 & NA & 46.9 & NA & NR & 21 & NA & $\geq 1$ & & & 95\% CI: & 0.0 & 0.0 \\
\hline & 10.23 & 0.00341 & NA & NR & 67 & NA & 15.1 & NA & NR & 67 & NA & $\geq 1$ & $\mathrm{LOD}_{95}$ & 0.038 & $(0.029,0.047)$ & 0.367 & 0.0 \\
\hline & 10.24 & 0.00586 & NA & NR & 32 & NA & 19.1 & NA & NR & 32 & NA & $\geq 1$ & $\mathrm{LOD}_{90}$ & 0.031 & NR & 0.074 & 0.0 \\
\hline
\end{tabular}


Table A.2b Expanded summary of test results for wipe sampling. Acronyms and abbreviations are defined in Table 6. (cont.)

\begin{tabular}{|c|c|c|c|c|c|c|c|c|c|c|c|c|c|c|c|c|c|}
\hline \multirow[b]{2}{*}{ Reference } & \multirow[b]{2}{*}{$\begin{array}{c}\text { Test } \\
\#\end{array}$} & \multicolumn{5}{|c|}{$\begin{array}{c}\text { Recovery concentration results - } \\
\text { Mean \& \%RSDs }\end{array}$} & \multicolumn{5}{|c|}{$\begin{array}{l}\text { Recovery efficiency }(\mathrm{RE})- \\
\text { Mean \& \%RSDs } \\
\end{array}$} & \multicolumn{6}{|c|}{ LOD, FNR, and FPR } \\
\hline & & $\begin{array}{c}\text { Mean } \\
\left(\mathrm{CFU} / \mathrm{cm}^{2}\right)\end{array}$ & $\begin{array}{l}\mathrm{Lab} \\
\% \mathrm{RSD}\end{array}$ & $\begin{array}{l}\text { Run } \\
\% \text { RSD }\end{array}$ & $\begin{array}{l}\text { Sample- } \\
\text { within-run } \\
\text { \%RSD }\end{array}$ & $\begin{array}{c}\text { Total } \\
\% \text { RSD }\end{array}$ & $\begin{array}{c}\mathrm{RE} \\
\text { mean } \\
(\%)\end{array}$ & $\begin{array}{l}\text { RE lab } \\
\% \text { RSD }\end{array}$ & $\begin{array}{l}\text { RE run } \\
\% \text { RSD }\end{array}$ & $\begin{array}{l}\text { RE sample- } \\
\text { within-run } \\
\% \text { RSD }\end{array}$ & $\begin{array}{c}\mathrm{RE} \\
\text { total } \\
\% \mathrm{RSD}\end{array}$ & $\begin{array}{c}\text { Positive } \\
\text { result } \\
\text { (CFU) }\end{array}$ & $\begin{array}{l}\text { LOD } \\
\text { defin- } \\
\text { ition }\end{array}$ & $\begin{array}{c}\text { LOD } \\
\left(\mathrm{CFU} / \mathrm{cm}^{2}\right)\end{array}$ & $\begin{array}{c}\text { LOD SD } \\
\text { or } 95 \% \mathrm{CI} \\
\left(\mathrm{CFU} / \mathrm{cm}^{2}\right)\end{array}$ & FNR & FPR \\
\hline \multirow{30}{*}{$\begin{array}{l}\text { Krauter et } \\
\text { al. } 2011 \\
\text { (cont.) }\end{array}$} & 10.25 & 0.00682 & NA & NR & 29 & NA & 18.3 & NA & NR & 29 & NA & $\geq 1$ & & & & 0.067 & 0.0 \\
\hline & 10.26 & 0.03548 & NA & NR & 18 & NA & 22.7 & NA & NR & 18 & NA & $\geq 1$ & & & & 0.0 & 0.0 \\
\hline & 10.27 & 0.46934 & NA & NR & 11 & NA & 25.3 & NA & NR & 11 & NA & $\geq 1$ & & & & 0.0 & 0.0 \\
\hline & 10.28 & 0.00031 & NA & NR & 316 & NA & 4.0 & NA & NR & 317 & NA & $\geq 1$ & & & & 0.933 & 0.0 \\
\hline & 10.29 & 0.00651 & NA & NR & 42 & NA & 42.4 & NA & NR & 42 & NA & $\geq 1$ & & & & 0.100 & 0.0 \\
\hline & 10.30 & 0.00837 & NA & NR & 30 & NA & 35.5 & NA & NR & 31 & NA & $\geq 1$ & & & & 0.033 & 0.0 \\
\hline & 10.31 & 0.00682 & NA & NR & 29 & NA & 29.8 & NA & NR & 29 & NA & $\geq 1$ & & & 95\% CI: & 0.033 & 0.0 \\
\hline & 10.32 & 0.00899 & NA & NR & 25 & NA & 27.4 & NA & NR & 26 & NA & $\geq 1$ & $\mathrm{LOD}_{95}$ & 0.018 & $(0.010,0.022)$ & 0.033 & 0.0 \\
\hline & 10.33 & 0.00930 & NA & NR & 35 & NA & 24.3 & NA & NR & 35 & NA & $\geq 1$ & $\mathrm{LOD}_{90}$ & 0.015 & NR & 0.033 & 0.0 \\
\hline & 10.34 & 0.01550 & NA & NR & 21 & NA & 28.5 & NA & NR & 21 & NA & $\geq 1$ & & & & 0.0 & 0.0 \\
\hline & 10.35 & 0.06076 & NA & NR & 28 & NA & 76.9 & NA & NR & 28 & NA & $\geq 1$ & & & & 0.0 & 0.0 \\
\hline & 10.36 & 0.08339 & NA & NR & 19 & NA & 54.3 & NA & NR & 19 & NA & $\geq 1$ & & & & 0.0 & 0.0 \\
\hline & 10.37 & 0.00124 & NA & NR & 129 & NA & 16.0 & NA & NR & 129 & NA & $\geq 1$ & & & & 0.733 & 0.0 \\
\hline & 10.38 & 0.00279 & NA & NR & 82 & NA & 18.2 & NA & NR & 82 & NA & $\geq 1$ & & & & 0.500 & 0.0 \\
\hline & 10.39 & 0.00713 & NA & NR & 21 & NA & 30.3 & NA & NR & 21 & NA & $\geq 1$ & & & & 0.0 & 0.0 \\
\hline & 10.40 & 0.00589 & NA & NR & 30 & NA & 25.7 & NA & NR & 30 & NA & $\geq 1$ & & & $95 \%$ CI: & 0.077 & 0.0 \\
\hline & 10.41 & 0.00341 & NA & NR & 52 & NA & 10.4 & NA & NR & 52 & NA & $\geq 1$ & $\mathrm{LOD}_{95}$ & 0.021 & $(0.018,0.024)$ & 0.333 & 0.0 \\
\hline & 10.42 & 0.00930 & NA & NR & 31 & NA & 24.3 & NA & NR & 31 & NA & $\geq 1$ & $\mathrm{LOD}_{90}$ & 0.023 & NR & 0.0 & 0.0 \\
\hline & 10.43 & 0.01798 & NA & NR & 16 & NA & 33.1 & NA & NR & 16 & NA & $\geq 1$ & & & & 0.0 & 0.0 \\
\hline & 10.44 & 0.03906 & NA & NR & 16 & NA & 49.4 & NA & NR & 16 & NA & $\geq 1$ & & & & 0.0 & 0.0 \\
\hline & 10.45 & 0.05642 & NA & NR & 19 & NA & 36.7 & NA & NR & 19 & NA & $\geq 1$ & & & & 0.0 & 0.0 \\
\hline & 10.46 & 0.0 & NA & NR & 0.0 & NA & 0.0 & NA & NR & 0.0 & NA & $\geq 1$ & & & & 1.0 & 0.0 \\
\hline & 10.47 & 0.00031 & NA & NR & 316 & NA & 2.0 & NA & NR & 316 & NA & $\geq 1$ & & & & 0.933 & 0.0 \\
\hline & 10.48 & 0.00155 & NA & NR & 105 & NA & 6.6 & NA & NR & 105 & NA & $\geq 1$ & & & & 0.67 & 0.0 \\
\hline & 10.49 & 0.00031 & NA & NR & 316 & NA & 1.4 & NA & NR & 316 & NA & $\geq 1$ & & & 95\% CI: & 0.933 & 0.0 \\
\hline & 10.50 & 0.00093 & NA & NR & 161 & NA & 2.8 & NA & NR & 161 & NA & $\geq 1$ & $\mathrm{LOD}_{95}$ & 0.051 & $(0.049,0.054)$ & 0.800 & 0.0 \\
\hline & 10.51 & 0.00527 & NA & NR & 40 & NA & 13.7 & NA & NR & 40 & NA & $\geq 1$ & $\mathrm{LOD}_{90}$ & 0.039 & NR & 0.200 & 0.0 \\
\hline & 10.52 & 0.00651 & NA & NR & 35 & NA & 12.0 & NA & NR & 35 & NA & $\geq 1$ & & & & 0.100 & 0.0 \\
\hline & 10.53 & 0.01395 & NA & NR & 24 & NA & 17.6 & NA & NR & 24 & NA & $\geq 1$ & & & & 0.0 & 0.0 \\
\hline & 10.54 & 0.03968 & NA & NR & 22 & NA & 25.8 & NA & NR & 22 & NA & $\geq 1$ & & & & 0.0 & 0.0 \\
\hline \multirow{6}{*}{$\begin{array}{l}\text { Rose et } \\
\text { al. } 2011\end{array}$} & 11.1 & 0.00636 & NR & NR & NR & NR & 46.1 & 48 & NA & 54 & 72 & $\geq 1$ & & & & 0.159 & 0.0 \\
\hline & 11.2 & 0.17469 & NR & NR & NR & NR & 66.5 & 30 & NA & 20 & 36 & $\geq 1$ & NR & NR & NR & 0.0 & 0.0 \\
\hline & 11.3 & 13.341 & NR & NR & NR & NR & 77.9 & 14 & NA & 11 & 18 & $\geq 1$ & & & & 0.0 & 0.056 \\
\hline & 11.4 & 0.01302 & NR & NR & NR & NR & 32.4 & 31 & NA & 69 & 76 & $\geq 1$ & & & & NR & NR \\
\hline & 11.5 & 0.20553 & NR & NR & NR & NR & 24.4 & 31 & NA & 35 & 47 & $\geq 1$ & NR & 0.031 & NR & NR & NR \\
\hline & 11.6 & 15.475 & NR & NR & NR & NR & 30.1 & 20 & NA & 20 & 28 & $\geq 1$ & & & & NR & NR \\
\hline
\end{tabular}


Table A.2b Expanded summary of test results for wipe sampling. Acronyms and abbreviations are defined in Table 6. (cont.)

\begin{tabular}{|c|c|c|c|c|c|c|c|c|c|c|c|c|c|c|c|c|c|}
\hline \multirow[b]{2}{*}{ Reference } & \multirow[b]{2}{*}{$\begin{array}{c}\text { Test } \\
\#\end{array}$} & \multicolumn{5}{|c|}{$\begin{array}{c}\text { Recovery concentration results - } \\
\text { Mean \& \%RSDs }\end{array}$} & \multicolumn{5}{|c|}{$\begin{array}{c}\text { Recovery efficiency }(\mathrm{RE})- \\
\text { Mean \& \%RSDs }\end{array}$} & \multicolumn{6}{|c|}{ LOD, FNR, and FPR } \\
\hline & & $\begin{array}{c}\text { Mean } \\
\left(\mathrm{CFU} / \mathrm{cm}^{2}\right) \\
\end{array}$ & $\begin{array}{l}\text { Lab } \\
\% \text { RSD }\end{array}$ & $\begin{array}{l}\text { Run } \\
\% \text { RSD }\end{array}$ & $\begin{array}{c}\text { Sample- } \\
\text { within-run } \\
\% \text { RSD }\end{array}$ & $\begin{array}{c}\text { Total } \\
\% \text { RSD }\end{array}$ & $\begin{array}{l}\mathrm{RE} \\
\text { mean } \\
(\%)\end{array}$ & $\begin{array}{l}\text { RE lab } \\
\text { \%RSD }\end{array}$ & $\begin{array}{l}\text { RE run } \\
\% \text { RSD }\end{array}$ & $\begin{array}{c}\text { RE sample- } \\
\text { within-run } \\
\% \text { RSD }\end{array}$ & $\begin{array}{c}\mathrm{RE} \\
\text { total } \\
\% \mathrm{RSD}\end{array}$ & $\begin{array}{c}\text { Positive } \\
\text { result } \\
(\mathrm{CFU})\end{array}$ & $\begin{array}{l}\text { LOD } \\
\text { defin- } \\
\text { ition }\end{array}$ & $\begin{array}{l}\text { LOD } \\
(\mathrm{CFU} / \\
\left.\mathrm{cm}^{2}\right) \\
\end{array}$ & $\begin{array}{c}\text { LOD SD } \\
\text { or } 95 \% \mathrm{CI} \\
\left(\mathrm{CFU} / \mathrm{cm}^{2}\right)\end{array}$ & FNR & FPR \\
\hline \multirow{5}{*}{$\begin{array}{l}\text { Rose et } \\
\text { al. } 2011 \\
\text { (cont.) }\end{array}$} & 11.7 & NR & NR & NR & NR & NR & 30.8 & NA & NA & 50 & NA & NR & NR & NR & NR & NR & NR \\
\hline & 11.8 & NR & NR & NR & NR & NR & 32.3 & NA & NA & 30 & NA & NR & NR & NR & NR & NR & NR \\
\hline & 11.9 & NR & NR & NR & NR & NR & 26.8 & NA & NA & 21 & NA & NR & NR & NR & NR & NR & NR \\
\hline & 11.10 & NR & NR & NR & NR & NR & 36.3 & NA & NA & 25 & NA & NR & NR & NR & NR & NR & NR \\
\hline & 11.11 & NR & $\mathrm{NR}$ & NR & NR & NR & 26.0 & NA & NA & 38 & NA & NR & NR & NR & NR & NR & NR \\
\hline \multirow{4}{*}{$\begin{array}{l}\text { Montgomery } \\
\text { and Camp } \\
2008\end{array}$} & 12.1 & NR & NA & NR & NR & NA & 19.9 & NA & 10 & 27 & NA & NR & NR & NR & NR & NR & NR \\
\hline & 12.2 & NR & NA & NR & NR & NA & 14.5 & NA & 21 & 0 & NA & NR & NR & NR & NR & NR & NR \\
\hline & 12.3 & NR & NA & NR & NR & NA & 15.7 & NA & 37 & 14 & NA & NR & NR & NR & NR & NR & NR \\
\hline & 12.4 & NR & NA & NR & NR & NA & 16.3 & NA & 26 & 12 & NA & NR & NR & NR & NR & NR & NR \\
\hline
\end{tabular}

(a) Combined estimate of "run" and "sample-within-run" uncertainties.

(b) Data were available to calculate "run" and "sample-within-run" uncertainties, but they were not reported by Einfeld et al. (2011). The uncertainty values reported are listed in the "RE run \%RSD" column, even though the values have contributions from "runs" as well as "samples within runs".

(c) Quizon et al. (2007) reported a SE = 7.6, but not enough information was provided to 1) know whether it includes run-to-run and/or sample-within-run uncertainties, and 2) convert the SE value to a $\%$ RSD value. 
Table A.3a Expanded summary of test conditions for vacuum sampling. Acronyms and abbreviations are defined in Table 6.

\begin{tabular}{|c|c|c|c|c|c|c|c|c|c|c|c|c|c|c|c|}
\hline Reference & $\begin{array}{c}\text { Test } \\
\# \\
\end{array}$ & Agent $^{(a)}$ & $\begin{array}{c}\text { Agent }^{(b)} \\
\text { deposition }\end{array}$ & $\begin{array}{c}\text { Agent }^{(\mathrm{c})} \\
\text { concentration } \\
\left(\mathrm{CFU} / \mathrm{cm}^{2}\right)\end{array}$ & $\begin{array}{l}\text { Vacuum } \\
\text { filter type }\end{array}$ & $\begin{array}{c}\text { Vacuum } \\
\text { tech- } \\
\text { nique }^{(a)}\end{array}$ & $\begin{array}{c}\text { Relative } \\
\text { humidity } \\
(\%)\end{array}$ & $\begin{array}{c}\text { Surface } \\
\text { type }\end{array}$ & $\begin{array}{l}\text { Surface } \\
\text { area } \\
\text { sampled } \\
\left(\mathrm{cm}^{2}\right) \\
\end{array}$ & $\begin{array}{c}\text { Extraction } \\
\text { liquid }\end{array}$ & $\begin{array}{c}\text { Extraction } \\
\text { method }\end{array}$ & $\begin{array}{l}\text { Culture } \\
\text { method, } \\
\text { medium }\end{array}$ & $\begin{array}{c}\# \\
\text { labs }\end{array}$ & $\begin{array}{l}\# \text { test } \\
\text { runs }\end{array}$ & $\begin{array}{r}\text { Total } \\
\text { \# test } \\
\text { samples }\end{array}$ \\
\hline Estill et & 1.1 & BA Sterne & Dry aerosol & 0.03 & HEPA sock & P2D & NR & SS & 929 & BBT & $\mathrm{A}+\mathrm{C}+\mathrm{V}+\mathrm{S}$ & FP, TSAB & 1 & 3 & 27 \\
\hline \multirow[t]{5}{*}{ al. 2009} & 1.2 & BA Sterne & Dry aerosol & 0.3 & HEPA sock & P2D & NR & SS & 929 & BBT & $\mathrm{A}+\mathrm{C}+\mathrm{V}+\mathrm{S}$ & $\mathrm{FP}, \mathrm{TSAB}$ & 1 & 3 & 27 \\
\hline & 1.3 & BA Sterne & Dry aerosol & 2 & HEPA sock & $\mathrm{P} 2 \mathrm{D}$ & NR & SS & 929 & BBT & $\mathrm{A}+\mathrm{C}+\mathrm{V}+\mathrm{S}$ & FP, TSAB & 1 & 3 & 27 \\
\hline & 1.4 & BA Sterne & Dry aerosol & 0.03 & HEPA sock & $\mathrm{P} 2 \mathrm{D}$ & NR & Carpet & 929 & BBT & $\mathrm{A}+\mathrm{C}+\mathrm{V}+\mathrm{S}$ & FP, TSAB & 1 & 2 & 18 \\
\hline & 1.5 & BA Sterne & Dry aerosol & 0.3 & HEPA sock & $\mathrm{P} 2 \mathrm{D}$ & NR & Carpet & 929 & BBT & $\mathrm{A}+\mathrm{C}+\mathrm{V}+\mathrm{S}$ & $\mathrm{FP}, \mathrm{TSAB}$ & 1 & 4 & 36 \\
\hline & 1.6 & BA Sterne & Dry aerosol & 2 & HEPA sock & P2D & NR & Carpet & 929 & BBT & $\mathrm{A}+\mathrm{C}+\mathrm{V}+\mathrm{S}$ & $\mathrm{FP}, \mathrm{TSAB}$ & 1 & 3 & 27 \\
\hline Brown et & 2.1 & $\mathrm{BA}$ & Dry aerosol & $1 \mathrm{E}+2-1 \mathrm{E}+3$ & HEPA filter & PP2D & $30-40$ & SS & 100 & BBT & $\mathrm{S}+\mathrm{H}+\mathrm{V}$ & Plate, PF & 1 & NR & 23 \\
\hline \multirow[t]{7}{*}{ al. $2007 \mathrm{c}$} & 2.2 & BA & Dry aerosol & $1 \mathrm{E}+4-1 \mathrm{E}+5$ & HEPA filter & PP2D & $30-40$ & SS & 100 & BBT & $\mathrm{S}+\mathrm{H}+\mathrm{V}$ & Plate, PF & 1 & NR & 13 \\
\hline & 2.3 & $\mathrm{BA}$ & Dry aerosol & $1 E+2-1 E+3$ & HEPA filter & PP2D & $30-40$ & PWB & 100 & BBT & $\mathrm{S}+\mathrm{H}+\mathrm{V}$ & Plate, PF & 1 & NR & 14 \\
\hline & 2.4 & BA & Dry aerosol & $1 E+4-1 E+5$ & HEPA filter & PP2D & $30-40$ & PWB & 100 & BBT & $\mathrm{S}+\mathrm{H}+\mathrm{V}$ & Plate, PF & 1 & NR & 22 \\
\hline & 2.5 & BA & Dry aerosol & $1 E+2-1 E+3$ & HEPA filter & PP2D & $30-40$ & Carpet & 100 & BBT & $\mathrm{S}+\mathrm{H}+\mathrm{V}$ & Plate, PF & 1 & NR & 16 \\
\hline & 2.6 & BA & Dry aerosol & $1 E+4-1 E+5$ & HEPA filter & PP2D & $30-40$ & Carpet & 100 & BBT & $\mathrm{S}+\mathrm{H}+\mathrm{V}$ & Plate, PF & 1 & NR & 24 \\
\hline & 2.7 & BA & Dry aerosol & $1 E+2-1 E+3$ & HEPA filter & PP2D & $30-40$ & Concrete & 100 & BBT & $\mathrm{S}+\mathrm{H}+\mathrm{V}$ & Plate, PF & 1 & NR & 21 \\
\hline & 2.8 & $\mathrm{BA}$ & Dry aerosol & $1 \mathrm{E}+4-1 \mathrm{E}+5$ & HEPA filter & PP2D & $30-40$ & Concrete & 100 & BBT & $\mathrm{S}+\mathrm{H}+\mathrm{V}$ & Plate, PF & 1 & NR & 23 \\
\hline \multirow{6}{*}{$\begin{array}{l}\text { Einfeld et } \\
\text { al. } 2011\end{array}$} & 3.1 & BAtr + grime & Dry aerosol & $1.42 \mathrm{E}+3-2.00 \mathrm{E}+4$ & PE filter & NR & $36-48$ & Marble & 100 & BBT & $\mathrm{S}+\mathrm{H}+\mathrm{V}$ & Plate, PF & 1 & 3 & 33 \\
\hline & 3.2 & BAtr & Dry aerosol & $1.42 \mathrm{E}+3-2.00 \mathrm{E}+4$ & PE filter & NR & $36-48$ & Marble & 100 & BBT & $\mathrm{S}+\mathrm{H}+\mathrm{V}$ & Plate, PF & 1 & 3 & 36 \\
\hline & 3.3 & BAtr + grime & Dry aerosol & $3.80 \mathrm{E}+3-8.78 \mathrm{E}+4$ & PE filter & NR & $77-82$ & Marble & 100 & BBT & $\mathrm{S}+\mathrm{H}+\mathrm{V}$ & Plate, PF & 1 & 3 & 36 \\
\hline & 3.4 & BAtr & Dry aerosol & $3.80 \mathrm{E}+3-8.78 \mathrm{E}+4$ & PE filter & NR & $77-82$ & Marble & 100 & BBT & $\mathrm{S}+\mathrm{H}+\mathrm{V}$ & Plate, PF & 1 & 3 & 35 \\
\hline & 3.5 & BAtr + grime & Dry aerosol & $1.31 \mathrm{E}+3-7.75 \mathrm{E}+3$ & PE filter & NR & $89-90$ & Concrete & 100 & BBT & $\mathrm{S}+\mathrm{H}+\mathrm{V}$ & Plate, PF & 1 & 5 & 59 \\
\hline & 3.6 & BAtr & Dry aerosol & $1.31 \mathrm{E}+3-7.75 \mathrm{E}+3$ & PE filter & NR & $89-90$ & Concrete & 100 & BBT & $\mathrm{S}+\mathrm{H}+\mathrm{V}$ & Plate, PF & 1 & 5 & 58 \\
\hline \multirow{6}{*}{$\begin{array}{l}\text { Quizon et } \\
\text { al. } 2007\end{array}$} & 4.1 & BAtr & Wet aerosol & $\mathrm{NR}$ & HEPA sock & $\mathrm{SVH}$ & NR & Ceiling tile & 900 & PBST & $\mathrm{C}+\mathrm{V}+\mathrm{S}$ & SP/FP, TSA & 1 & 4 & 6 \\
\hline & 4.2 & BAtr & Wet aerosol & NR & HEPA sock & SVH & NR & Carpet & 900 & PBST & $\mathrm{C}+\mathrm{V}+\mathrm{S}$ & SP/FP, TSA & 1 & 4 & 4 \\
\hline & 4.3 & BAtr & Wet aerosol & NR & HEPA sock & $\mathrm{SVH}$ & NR & PWB & 900 & PBST & $\mathrm{C}+\mathrm{V}+\mathrm{S}$ & SP/FP, TSA & 1 & 4 & 4 \\
\hline & 4.4 & BAtr & Wet aerosol & NR & HEPA sock & $\mathrm{SVH}$ & NR & SS & 900 & PBST & $\mathrm{C}+\mathrm{V}+\mathrm{S}$ & SP/FP, TSA & 1 & 4 & 10 \\
\hline & 4.5 & BAtr & Wet aerosol & NR & HEPA sock & $\mathrm{SVH}$ & NR & Vinyl & 900 & PBST & $\mathrm{C}+\mathrm{V}+\mathrm{S}$ & SP/FP, TSA & 1 & 4 & 4 \\
\hline & 4.6 & BAtr & Wet aerosol & NR & HEPA sock & SVH & NR & Wood & 900 & PBST & $\mathrm{C}+\mathrm{V}+\mathrm{S}$ & SP/FP, TSA & 1 & 4 & 4 \\
\hline \multirow{10}{*}{$\begin{array}{l}\text { Montgomery } \\
\text { and Camp } \\
2008\end{array}$} & 5.1 & BA Sterne & EtOH drops & 798.25 & HEPA sock & Rough & NR & HVAC filter & 309.68 & PBSTr & Shake+C & NR, NR & 1 & 3 & NR \\
\hline & 5.2 & BA Sterne & EtOH drops & 798.25 & TECF & Gentle & NR & HVAC filter & 309.68 & PBSTr & Shake $+\mathrm{C}$ & NR, NR & 1 & 3 & 9 \\
\hline & 5.3 & BA Sterne & EtOH drops & 798.25 & TECF & Rough & NR & HVAC filter & 309.68 & PBSTr & Shake $+\mathrm{C}$ & NR, NR & 1 & 3 & 9 \\
\hline & 5.4 & BA Sterne & EtOH drops & $9.0 \mathrm{E}+4$ & TECF & Rough & NR & HVAC filter & 309.68 & PBSTr & Shake $+\mathrm{C}$ & NR, NR & 1 & NR & NR \\
\hline & 5.5 & BA Sterne & EtOH drops & $9.1 \mathrm{E}+6$ & TECF & Rough & NR & HVAC filter & 309.68 & PBSTr & Shake $+\mathrm{C}$ & NR, NR & 1 & NR & NR \\
\hline & 5.6 & BA Sterne & EWD & 167 & TECF & V. slide & NR & Carpet & 400 & PBSTr & Shake $+\mathrm{C}$ & NR, NR & 1 & 1 & 3 \\
\hline & 5.7 & BA Sterne & EWD & 167 & TECF & Tilt drag & NR & Carpet & 400 & PBSTr & Shake $+\mathrm{C}$ & NR, NR & 1 & 1 & 3 \\
\hline & 5.8 & BA Sterne & EWD & 201 & TECF & V. slide & NR & Carpet & 400 & PBSTr & Shake $+\mathrm{C}$ & NR, NR & 1 & 1 & 6 \\
\hline & 5.9 & BA Sterne & $\mathrm{H} 2 \mathrm{O}$ drops & 111 & TECF & V. slide & NR & Carpet & 400 & PBSTr & Shake $+\mathrm{C}$ & NR, NR & 1 & 1 & 3 \\
\hline & 5.10 & BA Sterne & $\mathrm{H} 2 \mathrm{O}$ drops & 111 & TECF & Tilt push & NR & Carpet & 400 & PBSTr & Shake+C & NR, NR & 1 & 1 & 3 \\
\hline
\end{tabular}

(a) V. slide $=$ vertical slide 
Table A.3b Expanded summary of test results for vacuum sampling. Acronyms and abbreviations are defined in Table 6.

\begin{tabular}{|c|c|c|c|c|c|c|c|c|c|c|c|c|c|c|c|c|c|}
\hline \multirow[b]{2}{*}{ Reference } & \multirow[b]{2}{*}{$\begin{array}{c}\text { Test } \\
\#\end{array}$} & \multicolumn{5}{|c|}{$\begin{array}{c}\text { Recovery concentration results - } \\
\text { Mean \& \%RSDs } \\
\end{array}$} & \multicolumn{5}{|c|}{$\begin{array}{l}\text { Recovery efficiency (RE) - } \\
\text { Mean \& \%RSDs } \\
\end{array}$} & \multicolumn{6}{|c|}{ LOD, FNR, and FPR } \\
\hline & & $\begin{array}{c}\text { Mean } \\
\left(\mathrm{CFU} / \mathrm{cm}^{2}\right)\end{array}$ & $\begin{array}{l}\text { Lab } \\
\% \text { RSD }\end{array}$ & $\begin{array}{l}\text { Run } \\
\% \text { RSD }\end{array}$ & $\begin{array}{l}\text { Sample- } \\
\text { within-run } \\
\% \text { RSD }\end{array}$ & $\begin{array}{c}\text { Total } \\
\% \text { RSD }\end{array}$ & $\begin{array}{c}\mathrm{RE} \\
\text { mean } \\
(\%)\end{array}$ & $\begin{array}{l}\text { RE lab } \\
\text { \%RSD }\end{array}$ & $\begin{array}{l}\text { RE run } \\
\% \text { RSD }\end{array}$ & $\begin{array}{c}\text { RE sample- } \\
\text { within-run } \\
\% \text { RSD }\end{array}$ & \begin{tabular}{|c|}
$\mathrm{RE}$ \\
total \\
$\% \mathrm{RSD}$ \\
\end{tabular} & $\begin{array}{l}\text { Positive } \\
\text { result } \\
\text { (CFU) }\end{array}$ & $\begin{array}{l}\text { LOD } \\
\text { defin- } \\
\text { ition }\end{array}$ & $\begin{array}{c}\mathrm{LOD} \\
\left(\mathrm{CFU} / \mathrm{cm}^{2}\right)\end{array}$ & $\begin{array}{l}\text { LOD SD } \\
\text { or } 95 \% \text { CI } \\
\left(\mathrm{CFU} / \mathrm{cm}^{2}\right)\end{array}$ & FNR & FPR \\
\hline \multirow{5}{*}{$\begin{array}{l}\text { Estill et } \\
\text { al. } 2009\end{array}$} & 1.1 & 0.0043 & 38 & 50 & 98 & 116 & 5.5 & 16 & 5 & 90 & 92 & & & & & & \\
\hline & 1.2 & 0.018 & 18 & $\begin{array}{l}0 \\
6\end{array}$ & $\begin{array}{l}82 \\
82\end{array}$ & $\begin{array}{l}84 \\
89\end{array}$ & $\begin{array}{l}4.7 \\
3.7\end{array}$ & 13 & $\begin{array}{l}0 \\
0\end{array}$ & $\begin{array}{l}81 \\
73\end{array}$ & $\begin{array}{l}82 \\
79\end{array}$ & $\geq 3$ & $\mathrm{LOD}_{95}$ & 0.44 & $0.24-1.4$ & NR & $\begin{array}{l}8 / 30= \\
0.267\end{array}$ \\
\hline & 1.4 & 0.0011 & 0 & 0 & 170 & 170 & 6.3 & 0 & 0 & 130 & 130 & & & & & & \\
\hline & 1.5 & 0.0061 & 0 & 57 & 110 & 124 & 3.7 & 0 & 61 & 110 & 130 & $\geq 1$ & $\mathrm{LOD}_{95}$ & 0.28 & $95 \%$ Cl: & NR & $\begin{array}{l}2 / 30= \\
0067\end{array}$ \\
\hline & 1.6 & 0.062 & 30 & 37 & 44 & 65 & 4.7 & 18 & 89 & 50 & 100 & & & & & & \\
\hline \multirow{8}{*}{$\begin{array}{l}\text { Brown et } \\
\text { al. } 2007 \mathrm{c}\end{array}$} & 2.1 & NR & NA & NR & NR & NA & 32.1 & NA & NR & 45 & NA & $\geq 1 \mathrm{CFU} / \mathrm{ml}$ & NR & NR & NR & NR & NR \\
\hline & 2.2 & NR & NA & NR & NR & NA & 23.1 & NA & NR & 46 & NA & $\geq 1 \mathrm{CFU} / \mathrm{ml}$ & NR & NR & NR & NR & NR \\
\hline & 2.3 & NR & NA & NR & NR & $\mathrm{NA}$ & 24.5 & NA & NR & 78 & NA & $\geq 1 \mathrm{CFU} / \mathrm{ml}$ & NR & NR & NR & NR & NR \\
\hline & 2.4 & NR & NA & NR & NR & NA & 25.0 & NA & NR & 44 & NA & $\geq 1 \mathrm{CFU} / \mathrm{ml}$ & NR & NR & NR & NR & NR \\
\hline & 2.5 & NR & NA & NR & NR & NA & 36.1 & NA & NR & 47 & NA & $\geq 1 \mathrm{CFU} / \mathrm{ml}$ & NR & NR & NR & NR & NR \\
\hline & 2.6 & NR & NA & NR & NR & NA & 22.9 & NA & NR & 28 & NA & $\geq 1 \mathrm{CFU} / \mathrm{ml}$ & NR & NR & NR & NR & NR \\
\hline & 2.7 & NR & NA & NR & NR & NA & 21.6 & NA & NR & 90 & NA & $\geq 1 \mathrm{CFU} / \mathrm{ml}$ & NR & NR & NR & NR & NR \\
\hline & 2.8 & NR & NA & NR & NR & NA & 16.4 & NA & NR & 43 & NA & $\geq 1 \mathrm{CFU} / \mathrm{ml}$ & NR & NR & NR & NR & NR \\
\hline \multirow{6}{*}{$\begin{array}{l}\text { Einfeld et } \\
\text { al. } 2011\end{array}$} & 3.1 & NR & NA & NR & NR & NA & 10.0 & NA & NR & 123 & NA & NR & NR & NR & NR & NR & NR \\
\hline & 3.2 & NR & NA & NR & NR & NA & 11.9 & NA & NR & 129 & NA & NR & NR & NR & NR & NR & NR \\
\hline & 3.3 & NR & NA & NR & NR & NA & 17.0 & NA & NR & 50 & NA & NR & NR & NR & NR & NR & NR \\
\hline & 3.4 & NR & NA & NR & NR & NA & 12.1 & NA & NR & 57 & NA & NR & NR & NR & NR & NR & NR \\
\hline & 3.5 & NR & NA & NR & NR & NA & 19.7 & NA & NR & 53 & NA & NR & NR & NR & NR & NR & NR \\
\hline & 3.6 & NR & NA & NR & NR & NA & 16.5 & NA & NR & 50 & NA & NR & NR & NR & NR & NR & NR \\
\hline \multirow{6}{*}{$\begin{array}{l}\text { Quizon et } \\
\text { al. } 2007\end{array}$} & 4.1 & 5200 & NA & $9^{(\mathrm{a})}$ & (a) & NA & NR & NA & NR & NR & NA & NR & NR & NR & NR & NR & NR \\
\hline & 4.2 & 4600 & NA & $19^{\text {(a) }}$ & (a) & NA & NR & NA & NR & NR & NA & NR & NR & NR & NR & NR & NR \\
\hline & 4.3 & 4800 & NA & $18^{(\mathrm{a})}$ & (a) & NA & NR & NA & NR & NR & NA & NR & NR & NR & NR & NR & NR \\
\hline & 4.4 & 2670 & NA & $9^{(a)}$ & (a) & NA & 4.4 & NA & (b) & (b) & NA & NR & NR & NR & NR & NR & NR \\
\hline & 4.5 & 1225 & NA & $13^{(\mathrm{a})}$ & (a) & NA & NR & NA & NR & NR & NA & NR & NR & NR & NR & NR & NR \\
\hline & 4.6 & 890 & NA & $20^{(\mathrm{a})}$ & (a) & NA & NR & NA & NR & NR & NA & NR & NR & NR & NR & NR & NR \\
\hline \multirow{10}{*}{$\begin{array}{l}\text { Montgomery } \\
\text { and Camp } \\
2008\end{array}$} & 5.1 & NR & NA & NR & NR & NA & 2.42 & NA & 1.3 & 24 & NA & NR & NR & NR & NR & NR & NR \\
\hline & 5.2 & NR & NA & NR & NR & NA & 1.16 & NA & 28 & 25 & NA & NR & NR & NR & NR & NR & NR \\
\hline & 5.3 & NR & NA & NR & NR & NA & 2.85 & NA & 16 & 13 & NA & NR & NR & NR & NR & NR & NR \\
\hline & 5.4 & NR & NA & NR & NR & NA & 3.52 & NA & $26^{(\mathrm{a})}$ & (a) & NA & NR & NR & NR & NR & NR & NR \\
\hline & 5.5 & NR & NA & NR & NR & NA & 2.84 & NA & NR & NR & NA & NR & NR & NR & NR & NR & NR \\
\hline & 5.6 & NR & NA & NR & NR & NA & 0.48 & NA & NA & 60 & NA & NR & NR & NR & NR & NR & NR \\
\hline & 5.7 & NR & NA & NR & NR & NA & 0.20 & NA & NA & 10 & NA & NR & NR & NR & NR & NR & NR \\
\hline & 5.8 & NR & NA & NR & NR & NA & 0.41 & NA & NA & 37 & NA & NR & NR & NR & NR & NR & NR \\
\hline & 5.9 & NR & NA & NR & NR & NA & 2.26 & NA & NA & 18 & NA & NR & NR & NR & NR & NR & NR \\
\hline & 5.10 & NR & NA & NR & NR & NA & 1.69 & NA & NA & 32 & NA & NR & NR & NR & NR & NR & NR \\
\hline
\end{tabular}

(a) Combined estimate of "run" and "sample-within-run" uncertainties.

(b) Quizon et al. (2007) reported a SE = 1.1, but not enough information was provided to 1) know whether it includes run-to-run and/or sample-within-run sources of uncertainty, and 2) convert the SE value to a \%RD value. 
Table A.4a Expanded summary of test conditions for storage and stability tests. Acronyms and abbreviations are defined in Table 6.

\begin{tabular}{|c|c|c|c|c|c|c|c|c|c|c|c|c|c|c|c|c|c|}
\hline \multirow[b]{2}{*}{ Reference } & \multirow[b]{2}{*}{$\begin{array}{c}\text { Test } \\
\#\end{array}$} & \multirow[b]{2}{*}{ Agent (a) } & \multirow{2}{*}{$\begin{array}{c}\text { Agent } \\
\text { deposition } \\
\text { (b) }\end{array}$} & \multirow{2}{*}{$\begin{array}{c}\text { Agent } \\
\text { concen- } \\
\text { tration } \\
(\mathrm{CFU} / \mathrm{ml})\end{array}$} & \multirow{2}{*}{$\begin{array}{l}\text { Sampling } \\
\text { medium } \\
\text { type (b) }\end{array}$} & \multirow{2}{*}{$\begin{array}{l}\text { Wetting } \\
\text { agent } \\
\text { (b) }\end{array}$} & \multirow{2}{*}{$\begin{array}{l}\text { Relative } \\
\text { humidity }\end{array}$} & \multirow{2}{*}{$\begin{array}{c}\text { Surface } \\
\text { type \& area } \\
\text { sampled (b) }\end{array}$} & \multicolumn{3}{|c|}{ Storage conditions } & \multirow{2}{*}{$\begin{array}{l}\text { Extract- } \\
\text { ion } \\
\text { liquid } \\
\text { (a) }\end{array}$} & \multirow{2}{*}{$\begin{array}{l}\text { Extract- } \\
\text { ion } \\
\text { method } \\
\text { (a) }\end{array}$} & \multirow{2}{*}{$\begin{array}{l}\text { Culture } \\
\text { method, } \\
\text { medium }\end{array}$} & \multirow[b]{2}{*}{$\begin{array}{c}\# \\
\text { labs }\end{array}$} & \multirow[b]{2}{*}{$\begin{array}{l}\text { \# test } \\
\text { runs }\end{array}$} & \multirow{2}{*}{$\begin{array}{r}\text { Total } \\
\text { \# test } \\
\text { samples }\end{array}$} \\
\hline & & & & & & & & & $\begin{array}{l}\text { Additive } \\
\text { (c) }\end{array}$ & $\begin{array}{l}\text { Temp. } \\
\left({ }^{\circ} \mathrm{C}\right)\end{array}$ & $\begin{array}{c}\# \\
\text { Days }\end{array}$ & & & & & & \\
\hline Almeida & 1.1 & BA Sterne & Liquid & NR & NA & NA & NR & NA & None & 4 & 0 & NA & NA & Plate, LBA & NR & 3 lots & 19 \\
\hline \multirow[t]{19}{*}{ et al. 2008} & 1.2 & BA Sterne & Liquid & NR & NA & NA & NR & NA & None & 4 & 0 & NA & NA & Plate, LBA & NR & 1 & 3 \\
\hline & 1.3 & BA Sterne & Liquid & NR & NA & NA & NR & NA & None & 4 & 182 & NA & NA & Plate, LBA & NR & 1 & 3 \\
\hline & 1.4 & BA Sterne & Liquid & NR & NA & NA & NR & NA & None & 4 & 279 & NA & NA & Plate, LBA & NR & 1 & 3 \\
\hline & 1.5 & BA Sterne & Liquid & NR & NA & NA & NR & NA & Phenol & 4 & 0 & NA & NA & Plate, LBA & NR & 1 & 3 \\
\hline & 1.6 & BA Sterne & Liquid & NR & NA & NA & NR & NA & Phenol & 4 & 182 & NA & NA & Plate, LBA & NR & 1 & 3 \\
\hline & 1.7 & BA Sterne & Liquid & NR & NA & NA & NR & NA & Phenol & 4 & 279 & NA & NA & Plate, LBA & NR & 1 & 3 \\
\hline & 1.8 & BA Sterne & Liquid & NR & NA & NA & NR & NA & EDTA & 4 & 0 & NA & NA & Plate, LBA & NR & 1 & 3 \\
\hline & 1.9 & BA Sterne & Liquid & NR & NA & NA & NR & NA & EDTA & 4 & 182 & NA & NA & Plate, LBA & NR & 1 & 3 \\
\hline & 1.10 & BA Sterne & Liquid & NR & NA & NA & NR & NA & EDTA & 4 & 279 & NA & NA & Plate, LBA & NR & 1 & 3 \\
\hline & 1.11 & BA Sterne & Liquid & NR & NA & NA & NR & NA & Ethanol & 4 & 0 & NA & NA & Plate, LBA & NR & 1 & 3 \\
\hline & 1.12 & BA Sterne & Liquid & NR & NA & NA & NR & NA & Ethanol & 4 & 182 & NA & NA & Plate, LBA & NR & 1 & 3 \\
\hline & 1.13 & BA Sterne & Liquid & NR & NA & NA & NR & NA & Ethanol & 4 & 279 & NA & NA & Plate, LBA & NR & 1 & 3 \\
\hline & 1.14 & BA Sterne & Liquid & NR & NA & NA & NR & NA & PBSTr & 4 & 0 & NA & NA & Plate, LBA & NR & 1 & 3 \\
\hline & 1.15 & BA Sterne & Liquid & NR & NA & NA & NR & NA & PBSTr & 4 & 182 & NA & NA & Plate, LBA & NR & 1 & 3 \\
\hline & 1.16 & BA Sterne & Liquid & NR & NA & NA & NR & NA & PBSTr & 4 & 279 & NA & NA & Plate, LBA & NR & 1 & 3 \\
\hline & 1.17 & BA Sterne & Liquid & NR & NA & NA & NR & NA & None & -20 & 182 & NA & NA & Plate, LBA & NR & 1 & 3 \\
\hline & 1.18 & BA Sterne & Liquid & NR & NA & NA & NR & NA & None & -20 & 279 & NA & NA & Plate, LBA & NR & 1 & 3 \\
\hline & 1.19 & BA Sterne & Liquid & NR & NA & NA & NR & NA & None & -80 & 182 & NA & NA & Plate, LBA & NR & 1 & 3 \\
\hline & 1.20 & BA Sterne & Liquid & NR & NA & NA & NR & NA & None & -80 & 279 & NA & NA & Plate, LBA & NR & 1 & 3 \\
\hline
\end{tabular}

(a) The BA Sterne spores were diluted with PBS (10 mmol/L phosphate, $138 \mathrm{mmol} / \mathrm{L} \mathrm{NaCl}$, and $2.7 \mathrm{mmol} / \mathrm{L} \mathrm{KCl}$, $\mathrm{pH} 7.4)$ containing $0.01 \%$ Triton X-100, and vigorously mixed by vortexing.

(b) This study used only liquid samples containing the agent, so neither deposition onto surfaces nor sampling of surfaces was involved.

(c) Additives to sterile water: Ethanol $=$ ethanol $20 \%(\mathrm{v} / \mathrm{v})$, EDTA $=$ ethylendediamainetetraacetic acid, $10 \mathrm{mmol} / \mathrm{L}, \mathrm{pH} 8.0, \mathrm{Phenol}=\mathrm{phenol} 1 \%(\mathrm{v} / \mathrm{v}), \mathrm{PBSTr}=\mathrm{PBS}$ containing $0.01 \%(\mathrm{v} / \mathrm{v})$ Triton $\times 100$. 
Table A.4b Expanded summary of test results for storage and stability tests. Acronyms and abbreviations are defined in Table 6.

\begin{tabular}{|c|c|c|c|c|c|c|c|c|c|c|c|c|c|c|c|c|c|}
\hline \multirow[b]{2}{*}{ Reference } & \multirow[b]{2}{*}{$\begin{array}{c}\text { Test } \\
\#\end{array}$} & \multicolumn{5}{|c|}{ Recovery concentration results - Mean $\&$ \%RDs } & \multicolumn{5}{|c|}{ Recovery efficiency (RE) - Mean \& \%RSDs } & \multicolumn{6}{|c|}{ LOD, FNR, and FPR } \\
\hline & & $\begin{array}{c}\text { Mean } \\
(\mathrm{CFU} / \mathrm{ml})\end{array}$ & $\begin{array}{c}\mathrm{Lab} \\
\% \mathrm{RSD}\end{array}$ & $\begin{array}{c}\text { Run } \\
\% \text { RSD }\end{array}$ & $\begin{array}{c}\text { Sample- } \\
\text { within-run } \\
\% \mathrm{RSD}\end{array}$ & $\begin{array}{l}\text { Total } \\
\% \text { RSD }\end{array}$ & $\begin{array}{c}\mathrm{RE}^{(\mathrm{a})} \\
\text { mean } \\
(\%)\end{array}$ & $\begin{array}{l}\text { RE lab } \\
\% \text { RSD }\end{array}$ & $\begin{array}{l}\text { RE run } \\
\% \text { RSD }\end{array}$ & $\begin{array}{c}\text { RE sample- } \\
\text { within-run } \\
\% \text { RSD }\end{array}$ & $\begin{array}{c}\mathrm{RE} \\
\text { total } \\
\text { \%RSD }\end{array}$ & $\begin{array}{c}\text { Positive } \\
\text { result } \\
\text { (CFU) }\end{array}$ & $\begin{array}{l}\text { LOD } \\
\text { defin- } \\
\text { ition }\end{array}$ & $\begin{array}{c}\mathrm{LOD} \\
\left(\mathrm{CFU} / \mathrm{cm}^{2}\right)\end{array}$ & $\begin{array}{l}\text { LOD SD } \\
\text { or } 95 \% \mathrm{CI} \\
\left(\mathrm{CFU} / \mathrm{cm}^{2}\right)\end{array}$ & FNR & FPR \\
\hline \multirow{20}{*}{$\begin{array}{l}\text { Almeida } \\
\text { et al. } 2008\end{array}$} & 1.1 & $6.19 \mathrm{E}+9$ & NA & 174 & 25 & NA & 111 & NA & 22 & 24 & NA & NR & NR & NR & NR & NR & NR \\
\hline & 1.2 & $1.25 \mathrm{E}+8$ & NA & NA & 31 & NA & NA & NA & NA & NA & NA & NR & NR & NR & NR & NR & NR \\
\hline & 1.3 & $1.00 \mathrm{E}+8$ & NA & NA & 12 & NA & 81 & NA & NA & 33 & NA & NR & NR & NR & NR & NR & NR \\
\hline & 1.4 & $1.17 \mathrm{E}+8$ & NA & NA & 7 & NA & 96 & NA & NA & 30 & NA & NR & NR & NR & NR & NR & NR \\
\hline & 1.5 & $1.42 \mathrm{E}+8$ & NA & NA & 7 & NA & NA & NA & NA & NA & NA & NR & NR & NR & NR & NR & NR \\
\hline & 1.6 & $1.12 \mathrm{E}+8$ & NA & NA & 17 & NA & 79 & NA & NA & 18 & NA & NR & NR & NR & NR & NR & NR \\
\hline & 1.7 & $1.29 \mathrm{E}+8$ & NA & NA & 7 & NA & 92 & NA & NA & 10 & NA & NR & NR & NR & NR & NR & NR \\
\hline & 1.8 & $1.47 \mathrm{E}+8$ & NA & NA & 86 & NA & NA & NA & NA & NA & NA & NR & NR & NR & NR & NR & NR \\
\hline & 1.9 & $1.08 \mathrm{E}+8$ & NA & NA & 6 & NA & 71 & NA & NA & 55 & NA & NR & NR & NR & NR & NR & NR \\
\hline & 1.10 & $1.20 \mathrm{E}+8$ & NA & NA & 20 & NA & 75 & NA & NA & 54 & NA & NR & NR & NR & NR & NR & NR \\
\hline & 1.11 & $1.47 \mathrm{E}+8$ & NA & NA & 4 & NA & NA & NA & NA & NA & NA & NR & NR & NR & NR & NR & NR \\
\hline & 1.12 & $1.24 \mathrm{E}+8$ & NA & NA & 15 & NA & 85 & NA & NA & 16 & NA & NR & NR & NR & NR & NR & NR \\
\hline & 1.13 & $1.32 \mathrm{E}+8$ & NA & NA & 4 & NA & 90 & NA & NA & 6 & NA & NR & NR & NR & NR & NR & NR \\
\hline & 1.14 & $1.95 \mathrm{E}+8$ & NA & NA & 43 & NA & NA & NA & NA & NA & NA & NR & NR & NR & NR & NR & NR \\
\hline & 1.15 & $1.25 \mathrm{E}+8$ & NA & NA & 8 & NA & 71 & NA & NA & 45 & NA & NR & NR & NR & NR & NR & NR \\
\hline & 1.16 & $1.37 \mathrm{E}+8$ & NA & NA & 6 & NA & 77 & NA & NA & 43 & NA & NR & NR & NR & NR & NR & NR \\
\hline & 1.17 & $1.61 \mathrm{E}+8$ & NA & NA & 84 & NA & NA & NA & NA & NA & NA & NR & NR & NR & NR & NR & NR \\
\hline & 1.18 & $1.28 \mathrm{E}+8$ & NA & NA & 21 & NA & NA & NA & NA & NA & NA & NR & NR & NR & NR & NR & NR \\
\hline & 1.19 & $1.37 \mathrm{E}+8$ & NA & NA & 39 & NA & NA & NA & NA & NA & NA & NR & NR & NR & NR & NR & NR \\
\hline & 1.20 & $1.20 \mathrm{E}+8$ & NA & NA & 7 & NA & NA & NA & NA & NA & NA & NR & NR & NR & NR & NR & NR \\
\hline
\end{tabular}

(a) For Test 1.1, the REs at different storage times were calculated relative to the values for 0 days storage for each of three lots. Results did not appear to depend on storage time or lot, so the RE Mean was calculated over all lots and storage times. For Tests 1.2 to 1.20, RE could not be calculated using the data from 0-, 189-, and 279-day tests relative to the "true" concentration for each test, because that value was not reported by Almeida et al. (2008). For Tests 1.2 to 1.16, having several additives combined with spore solutions and stored for 0,182 , and 279 days, the RE mean values for 182 and 279 days were calculated relative to the results at 0 days. Hence, RE shows the effect of storage time on recovery of spores for those tests, although the effects of the plating and counting steps on RE are not included. 



\section{Distribution}

No. of

Copies

\section{External Distribution}

6 Department of Homeland Security Lance Brooks (lance.brooks@dhs.gov) Bert Coursey (bert.coursey@dhs.gov) Randy Long (randolph.long@dhs.gov) Kristin Pasternak (kristin.pasternak@associates.dhs.gov) Segaran Pillai (segaran.pillai@dhs.gov) Chris Russell (Christopher.E.Russell@dhs.gov)

8 Environmental Protection Agency Erica Canzler (canzler.erica@epa.gov) Schatzi Fitz-James (fitz-james.schatzi@epa.gov) Romy Lee (lee.romy@epa.gov) Dino Mattorano (mattorano.dino@epa.gov) Marissa Mullins (mullins.marissa@epa.gov) Tonya Nichols (nichols.tonya@epamail.epa.gov) Shawn Ryan (ryan.shawn@epa.gov)

Sanjiv Shah (shah.sanjiv@epa.gov)

3 Sandia National Laboratories Wayne Einfeld (weinfel@sandia.gov) Bob Knowlton (rgknowl@sandia.gov) Paula Krauter (pkraute@sandia.gov)

3 National Institute of Standards \& Technology Stuart Dols (wsdols@nist.gov) Jayne Morrow (jayne.morrow@nist.gov) Andrew Persily (andrew.persily@nist.gov)

2 Johns Hopkins University Applied Physics Laboratory Rachel Quizon (rachel.quizon@jhuapl.edu) Eric Van Gieson (eric.van.gieson@jhuapl.edu)

1 American College of Forensic Examiners John Bridges (JHBridges@vzw.blackberry.net)

3 Others Mark Buttner (buttner@unlv.nevada.edu) David Camp (camp2@llnl.gov) Jason Edmonds (jason.edmonds1@us.army.mil)
No. of

Copies

9 Centers for Disease Control and Protection Matthew Arduino (marduino@cdc.gov)

Lisa Delaney (Idelaney1@cdc.gov)

Cherie Estill (clf4@cdc.gov)

Laura Jevitt (lgj9@cdc.gov)

Richard Kellogg (rbk1@cdc.gov)

Stephen Morse (sam1@cdc.gov)

Laura Rose (lmr8@cdc.gov)

Angela Weber (aweber@cdc.gov)

Betsy Weirich (eweirich@cdc.gov)

4 Federal Bureau of Investigation

Doug Anders (douglas.anders@ic.fbi.gov)

Kristine Beardsley

(kristine.beardsley@ic.fbi.gov)

Doug Beecher (douglas.beecher@ic.fbi.gov)

Nick Paquette (nicholas.paquette@ic.fbi.gov)

2 Institute for Defense Analyses Jeff Grotte (jgrotte@ida.org)

Margaret Hebner (mhebner@ida.org)

4 Homeland Security Institute Suzanne Fleury (Suzanne.Fleury@hsi.dhs.gov) Phil Hammar (philip.hammar@hsi.dhs.gov) Ed Hildebrand (carl.hildebrand@hsi.dhs.gov) Eric Sylwester (eric.sylwester@hsi.dhs.gov)

1 Department of Defense - Joint Program Executive Office (JPEO)

Emma Wilson (emma.wilson@jpeocbd.osd.mil)

1 Department of Defense-ECBC Vipin Rastogi (vipin.rastogi@us.army.mil)

7 Internal Distribution

Pacific Northwest National Laboratory Brett Amidan (brett.amidan@pnnl.gov)

Brett Matzke (brett.matzke@pnnl.gov)

Greg Piepel (greg.piepel@pnnl.gov)

Brent Pulsipher (brent.pulsipher@pnnl.gov)

Landon Sego (landon.sego@pnnl.gov)

Nancy Valentine (nancy.valentine@pnnl.gov)

Information Release 


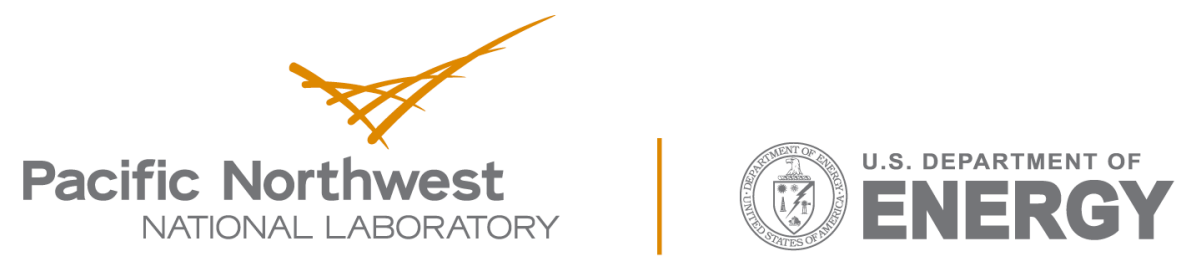

Proudly Operated by Battelle Since 1965

902 Battelle Boulevard

P.O. Box 999

Richland, WA 99352

1-888-375-PNNL (7665)

www.pnnl.gov 